\title{
Manipulation-resistant false-name-proof facility location mechanisms for complex graphs
}

\author{
Ilan Nehama ${ }^{1,3}$ (D) Taiki Todo $^{2}$ (D) $\cdot$ Makoto Yokoo $^{2}$ (D)
}

Accepted: 1 September 2021 / Published online: 22 January 2022

(c) The Author(s) 2022

\begin{abstract}
In many real-life scenarios, a group of agents needs to agree on a common action, e.g., on a location for a public facility, while there is some consistency between their preferences, e.g., all preferences are derived from a common metric space. The facility location problem models such scenarios and it is a well-studied problem in social choice. We study mechanisms for facility location on unweighted undirected graphs that are resistant to manipulations (strategy-proof, abstention-proof, and false-name-proof) by both individuals and coalitions on one hand and anonymous and efficient (Pareto-optimal) on the other. We define a new family of graphs, $Z V$-line graphs, and show a general facility location mechanism for these graphs that satisfies all these desired properties. This mechanism can also be computed in polynomial time and it can equivalently be defined as the first Pareto-optimal location according to some predefined order. Our main result, the $Z V$-line graphs family and the mechanism we present for it, unifies all works in the literature of false-name-proof facility location on discrete graphs including the preliminary (unpublished) works we are aware of. In particular, we show mechanisms for all graphs of at most five vertices, discrete trees, bicliques, and clique tree graphs. Finally, we discuss some generalizations and limitations of our result for facility location problems on other structures: Weighted graphs, large discrete cycles, infinite graphs; and for facility location problems concerning infinite societies.
\end{abstract}

Keywords Facility location $\cdot$ Strategy-proofness $\cdot$ False-name-proofness $\cdot Z V$-line graphs

\footnotetext{
A preliminary version appeared in the proceedings of AAMAS 2019, the 18th International Conference on Autonomous Agents and Multiagent Systems, under the title "Manipulation-resistant facility location mechanisms for ZV-line graphs" [49]

This work initialized while the first author was a member of the Multi-agent laboratory managed by the third author at Kyushu University, Japan

This work was partially supported by Israel Science Foundation Grant \#1626/18, JSPS KAKENHI Grants \#JP17H00761, \#JP17H04695, \#JP20H00587, \#JP20H00609, and JST SICORP JPMJSC1607.
}

I. Nehama: Main contributor.

Ilan Nehama

ilan.nehama@mail.huji.ac.il

Extended author information available on the last page of the article 


\section{Introduction}

Reaching an agreement could be hard. The seminal works of Gibbard [30] and Satterthwaite [59] show that one cannot devise a general procedure for aggregating the preferences of strategic agents to a single outcome, besides trivial procedures that a-priori ignore all agents except one (that is, the outcome is based on the preference of a predefined agent) or a-priori rule out all outcomes except two (that is, regardless of the preferences of the agents, the outcome is one of two predefined outcomes). The problem is that agents might act strategically, aiming to get an outcome that they prefer, so there might be scenarios in which for any profile of actions (a possible agreement) at least one of the agents prefers changing her action. Note that while we refer to a procedure and later to a mechanism, this impossibility is not technical but conceptual. We identify a procedure with the conceptual mapping induced by the procedure from the opinions of the agents to an agreement, while the procedure itself could be complex and abstract, e.g., to have several rounds or include a deliberation process between the agents (cheap-talk). For simplicity of terms, we refer to the direct mechanism that implements this mapping. That is, we think of an exogenous entity, the designer, who receives as input the opinions of the agents and returns as output the aggregated decision. This assumption does not hurt the generality, as according to the revelation principle [46], any general procedure is equivalent (w.r.t. the properties we study) to such a direct mechanism.

In many natural scenarios, it is exogenously given that the preferences satisfy some additional rationality property. Hence, the mechanism need not be defined for any profile of preferences, giving rise to mechanisms that are not prone to the above drawbacks. Two prominent examples are Vickrey-Clarke-Groves (VCG) mechanisms and generalizedmedian mechanisms. VCG mechanisms $[12,32,55,72]$ are the mechanisms that are resistant to manipulations like the ones described above for scenarios in which the preferences of agents are quasi-linear with respect to money [41, Def. 3.b.7], ${ }^{1}$ and monetary transfers are allowed (that is, the outcome space is closed under monetary exchanges between the agents or between the agents and the designer). The second example, Generalized-median mechanisms, does not include monetary transfers and has more of an ordinal flavor. Generalized-median mechanisms [42] are the mechanisms that are resistant to manipulations like above when it is known that the preferences are single-peaked w.r.t. the real line [5]. That is, the outcomes are locations on the real line and each agent has a unique optimal location, $\ell^{\star}$, on the line. The preference of an agent over the locations to the right of $\ell^{\star}$ is derived by the proximity to $\ell^{\star}$, and the preference over the locations to the left of $\ell^{\star}$ is derived similarly. For example, in the Euclidean single-peaked case, the preferences for all agents are minimizing the distance to their respective optimal locations.

\subsection{The facility location problem}

A natural generalization of the second scenario is the facility location problem. In this problem, we are given a metric space over the outcomes (that is, a distance function between outcomes) and it is assumed that the preference of each of the agents is defined

\footnotetext{
1 In a recent work, Nehama [47, 48] extended this result also to a family of non quasi-linear, almost quasilinear in disguise utilities, which are roughly preferences which are equivalent to a utility that is quasilinear in a function of the payment in some subset of the outcomes domain.
} 
by the distance to her optimal outcome: An agent with an optimal outcome $\ell^{\star}$ prefers an outcome $a$ over an outcome $b$ if and only if $a$ is closer to $\ell^{\star}$ than $b$. For ease of presentation, throughout this paper, we assume that there are finitely many agents and finitely many locations, and in Sect. 7.1 discuss the extension to the infinite case. In the finite case, a natural way to represent the metric space is using a weighted undirected graph. That is, having a vertex (location) for each outcome and weighted edges between vertices s.t. the distance between any two locations equals the distance between the two respective vertices (or generally to the length of the shortest path between them). Roughly speaking, given such a graph one seeks to find a mechanism that on one hand does not a-priori ignore some of the agents or rule out some of the locations, and on the other hand, is resistant to manipulations of the agents. Facility location problems and, moreover, facility location problems in which the underlying distance metric is a non-trivial combinatorial structure model many real-life scenarios of group decision making in which it is natural to assume some homogeneity between the preferences of different agents (e.g., an additional rationality assumption). These examples include not only locating a common facility, like a school, a bus stop, or a library, but also more general agreement scenarios with a common metric, e.g., a partition of a common budget to several tasks, committee selection, or group decision making with multi-dimensional criteria. Following the story of the facility location problem, we sometimes refer to the outcome of the mechanism as the facility. In this work, we look for mechanisms that satisfy the following desired properties:

Anonymity: The mechanism should not a-priori ignore agents and, moreover, we desire it to treat the agents equally in the following strong sense. The mechanism should be a function of the agents' votes (which we also refer to as ballots) but not their identities. Formally, the outcome of the mechanism should be invariant to any permutation of the ballots. In practice, most voting systems satisfy this property by first accumulating the different (physical) ballots, thus losing the agents' identities, and next applying the mechanism on the identity-less ballots.

We would also like the mechanism to treat the locations in an a-priori fair manner. Because of the inherent asymmetry induced by the graph, it is unreasonable to require that all locations are treated equally (i.e., neutrality of the mechanism). Instead, we require the following much weaker property of non-imposition.

Non-imposition/citizen sovereignty [1, 44]: The mechanism should not a-priori rule out a location, and each location should be the outcome of some profile. Formally, the mapping to a facility location should be an onto function.

Furthermore, the mechanism should respect the preferences of the agents and aim to optimize the aggregated welfare of the agents.

Pareto-optimality: The mechanism should not return a location $\ell$ if there exists another location $\ell^{\prime}$ s.t. switching from $\ell$ to $\ell^{\prime}$ benefits one of the agents (move the facility closer to her) while not hurting any of the other agents. In particular, if there exists a unique location that is unanimously most-preferred by all agents, then it must be the outcome. Note that any reasonable notion of aggregated welfare optimization entails Pareto-optimality.

Strategy-proofness: An agent should not be able to change the outcome to a location she strictly prefers by reporting a location different from her true location.

Abstention-proofness: ${ }^{2}$ An agent should not be able to change the outcome to a location she strictly prefers by not casting a ballot.

\footnotetext{
${ }^{2}$ In the voting literature (e.g., $\left.[26,43,75]\right)$ this property is also referred to as voluntary participation and the no-show paradox. This property is also equivalent to individual-rationality which takes the different point of view of mechanism design.
} 
False-name-proofness: An agent should not be able to change the outcome to a location she strictly prefers by casting more than one ballot. ${ }^{3}$ False-name manipulations received less attention in the classic social choice literature since in most voting scenarios there exists a central authority that can enforce a 'one person, one vote' principle (but cannot enforce participation or truthful voting). In contrast, many of the voting and aggregation scenarios nowadays are run in a distributed manner on some network and include virtual identities or avatars, which can be easily generated, so a manipulation of an agent pretending to represent many voters is eminent.

Resistance to group manipulations: We also consider a generalization of the above three properties dealing with manipulations of a coalition of agents. We define the preference of a coalition as the unanimous preference of its members. That is, a coalition $C$ weakly prefers an outcome $a$ over an outcome $b$ if all the agents in $C$ weakly prefer $a$ over $b$. Equivalently, $C$ strictly prefers $a$ over $b$ if

(i) all the agents in $C$ weakly prefer $a$ over $b(C$ weakly prefers $a$ over $b)$, and

(ii) at least one agent in $C$ strictly prefers $a$ over $b$ ( $C$ does not weakly prefer $b$ over $a$ ).

We require that a coalition should not be able to change the outcome to a location it strictly prefers by its members casting untruthful ballots, abstaining, or casting more than one ballot. We note that for onto mechanisms this property entails Pareto-optimality. Nevertheless, we prefer to think of Pareto-optimality as an efficiency requirement and not as a manipulation-resistance requirement.

\subsection{Our contribution}

In this paper, we define a family of unweighted undirected graphs, which we name $Z V$-line graphs, and show a general mechanism for facility location over these graphs that satisfies the desired properties. Roughly speaking, in a $Z V$-line graph there are two categories of locations, $Z$-locations and $V$-locations (and we also refer to them as $Z$-vertices and $V$-vertices), and the facility is commonly (except if all agents unanimously agree differently) positioned on a $Z$-location. ${ }^{4}$ For instance, the $Z$-locations could represent commercial locations for locating a public mall, or the set of status-quo outcomes. The mechanism is Paretooptimal and in particular, it satisfies citizen sovereignty and does not a-priori rule out any location; It is anonymous, so in particular, no agent is ignored; But on the other hand, it is resistant to all the above manipulations. Our mechanism for the $Z V$-line graphs family unifies the few mechanisms that are known and it induces mechanisms for many other graphs. ${ }^{5}$ To the best of our knowledge, this is the first work to show a general false-name-proof mechanism for a general family of graphs.

\footnotetext{
3 In the literature (e.g., [15]) one could also find a more restrictive definition of false-name-proofness in which the mechanism needs to be resistant only to an agent casting false-name ballots in addition to her truthful ballot.

4 The categorization is a combinatorial property of the graph (metric space). An agent can be located on any of the two categories.

5 Besides the work of Todo et al. [67], who characterized the false-name-proof mechanisms for facility location on the continuous line and on continuous trees, we are not aware of any other publications characterizing false-name-proof mechanisms for facility location on graphs which precedes our AAMAS publication [49] that. Moreover, as far as we know, a false-name-proof mechanism is known to the community only for very few simple graphs, and the current knowledge is still highly preliminary.
} 
Below, we show several common elementary graphs that are $Z V$-line graphs to demonstrate the richness and naturality of this family. The full formal definition of $Z V$-line graphs is given in Sects. 3 and 4, and we describe more examples for $Z V$-line graphs in Sects. 3.1 and 4.1. Consider the following family of graphs (which is a sub-family of the $Z V$-line graphs family and captures the gist of our mechanism). Let $\mathcal{G}=\langle\mathcal{V}, \mathcal{E}\rangle$ be a bipartite unweighted undirected graph with a vertex set $\mathcal{V}$ and an edge set $\mathcal{E}$. That is, there exists a partition of the vertices ${ }^{6} \mathcal{V}=V \dot{\cup} Z$ s.t. there are no edges between $V$-locations and no edges between $Z$-locations. We require that

(a) There exists a predefined order over the $Z$-locations, which we refer to as a left-toright order.

(b) Any of the $V$-locations is connected to a contiguous sequence of $Z$-locations.

Similar to the single-peaked consistency case [5], one can think of this constraint as a homogeneity constraint over the preferences of agents, i.e., as representing a restriction over the possible preference profiles, focusing on scenarios where voters' preferences are derived from some common structure. Our mechanism for these graphs:

The mechanism returns the leftmost Pareto-optimal $Z$-location if one exists. ${ }^{7}$

If no location in $Z$ is Pareto-optimal, then necessarily all agents voted for the same location, and the mechanism returns this location.

For example, bicliques (full bipartite graphs) can be represented as a $Z V$-line graph in which each $V$-location is connected to all the $Z$-locations (For example, Fig. 1). We use below $\bigcirc$ for $Z$-locations and $\checkmark$ for $V$-locations).

Our mechanism for biclique graphs:

$>$ If all agents voted unanimously for the same location, the mechanism returns this location.

If all agents voted for $V$-locations, the mechanism returns the leftmost $Z$-location.

Otherwise, the mechanism returns the leftmost $Z$-location that was voted for.

Notice that in this case, the order over the Z-locations is arbitrary (as well as the choice of one of the sides to be the Z-locations) in the sense that it is not derived from the graph but is a parameter of the mechanism. For instance, the order might represent the social norm of the society.

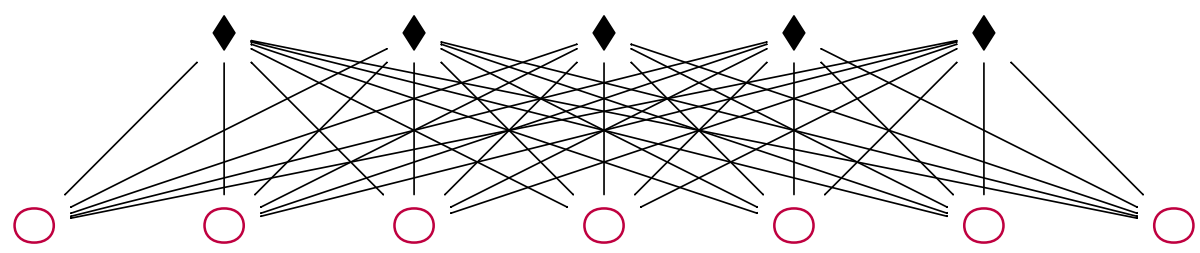

Fig. 1 The (5, 7)-biclique (Color figure online)

\footnotetext{
${ }^{6}$ We use the disjoint union notation $A \dot{\cup} B$ in cases we would like to emphasize that the sets $A$ and $B$ are disjoint.

7 An outcome $o \in \mathcal{V}$ is Pareto-optimal if there exists no location $o^{\prime} \in \mathcal{V}$ s.t. switching the outcome to be $o^{\prime}$ benefits one of the agents while not hurting any of the other agents.
} 


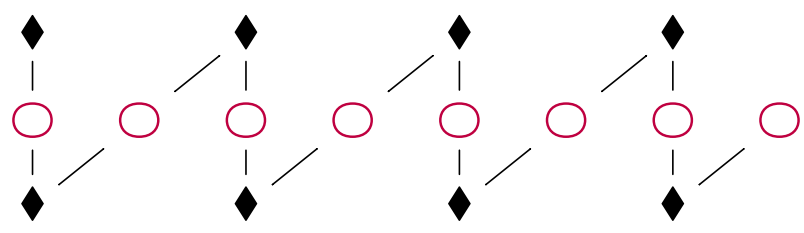

Fig. 2 The line graph of 16 locations (Color figure online)

A second example is the discrete line graph, which can be represented as a $Z V$-line graph in which every two consecutive $Z$-locations are connected by a unique $V$-location (For example, Fig. 2).

Using this representation we can show strategy-proof, false-name-proof, anonymous, Pareto-optimal facility location mechanisms for the discrete line that are distinct from the characterization of these mechanisms for the continuous line of Todo et al. [67]. Todo et al. [67, Thm. 2] showed that the strategy-proof, false-name-proof, anonymous, Pareto-optimal facility mechanisms for the continuous line are the mechanisms that return the closest Pareto-optimal location to some predefined location, which are a subfamily of the generalized median mechanisms. The mechanism for the discrete line that corresponds to the above representation as a $Z V$-line graph is:

If all agents voted unanimously for the same location, the mechanism returns this location.

If the leftmost ballot (according to the order of the line) is a $V$-location, the mechanism returns the $Z$-location to its right.

If the leftmost ballot is a $Z$-location, the mechanism returns this location.

In particular, this mechanism commonly returns one of the Z-locations, which constitute only fifty percent of the vertices.

Two elementary graphs that are generalizations of (the $Z V$-line graph representation of) the discrete line graph can be seen in Fig. 3 in which every two consecutive $Z$-locations are connected by two $V$-locations, and the $2 \times n$ grid (For example, see Fig. 4) which can be represented as a $Z V$-line graph in which every three consecutive $Z$-locations are connected by a unique $V$-location (see Fig. 5).

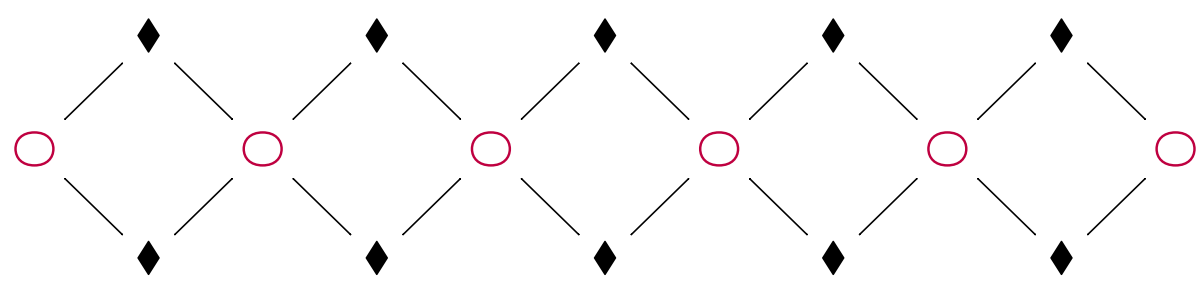

Fig. 3 A generalization of the discrete line graph (Color figure online)

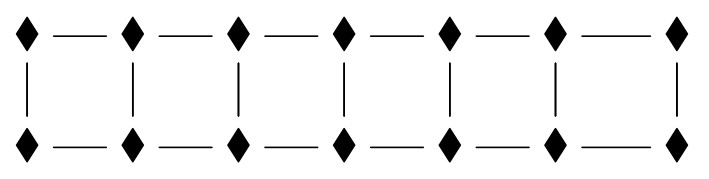

Fig. 4 The $2 \times 7$ grid 


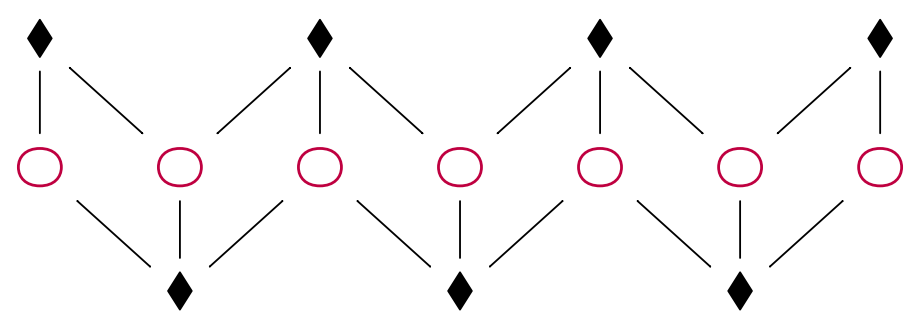

Fig. 5 The $2 \times 7$ grid (Color figure online)

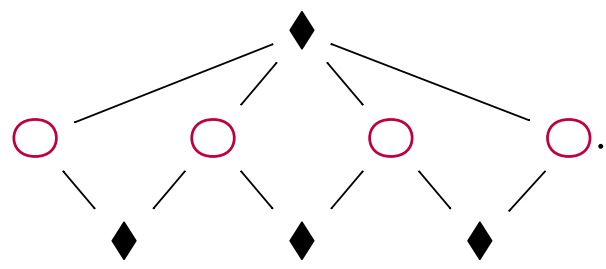

Fig. 6 Representing as a $Z V$-line graph (Color figure online)

A common property to all the above examples is their regularity. All the $V$-locations have the same degree and all the $Z$-locations have the same degree. An example we encountered of a non-regular graph for which a mechanism exists is which can be represented as a non-regular bipartite $Z V$-line graph as can be seen in Fig. 6 .

In the definition of the $Z V$-line graphs family (Definition 5) we extend the above family (and extend the mechanism accordingly) in two steps:

- First, we extend it to define simple $Z V$-line graphs (Definition 3) by allowing edges between $Z$-locations under a connectivity constraint, similar to the connectivity constraint we had on edges between $V$-locations and $Z$-locations. A $Z$ -location can be connected (in addition to $V$-locations as above), to a consecutive sequence of $Z$-locations to its immediate right and a consecutive sequence of $Z$ -locations to its immediate left (and any of the sequences might be empty).

- Second, replacing any location in a $Z$-line graph by a tree, a clique, or any other $\mathrm{ZV}$-line graph.

For example, the $Z V$-line graph in Fig. 7 is the outcome of taking a graph of the type of Fig. 3 and (See also Fig. 12 and the description next to it)

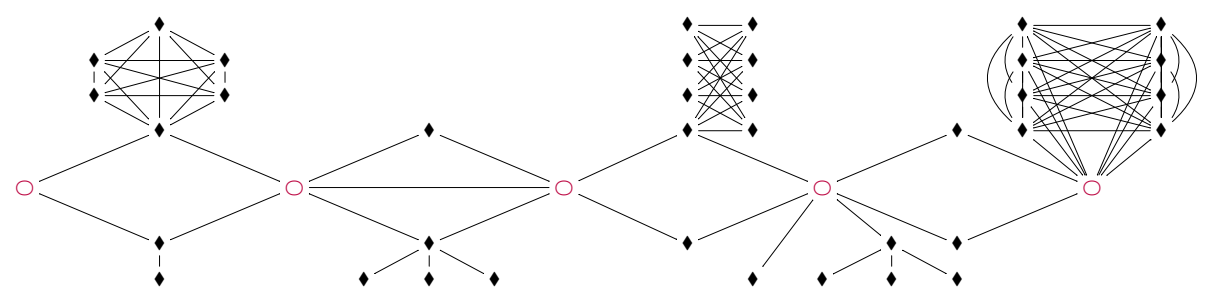

Fig. 7 A (non-simple) $Z V$-line graph (Color figure online) 
(a) adding an edge between the second and third Z-locations,

(b) replacing some of the locations by cliques, trees, and bicliques, which are $Z V$-line graphs.

In particular, as we discuss in Sects. 3.1 and 4.1, the $Z V$-line graphs family includes natural families like trees, cliques, bicliques, and block graphs [33], as well as all connected graphs of at most five locations (except the cycle of five locations) and all graphs for which (as far as we found) a false-name-proof mechanism was known to the community.

Last, we discuss two families of graphs: discrete cycles and recursive $Z V$-line graphs, that is, $Z V$-line graphs that are defined via a recursive construction, e.g., trees, cliques, and block graphs. We show that for recursive $Z V$-line graphs, a recursive simple mechanism is easily derived from our result. We show that there are no group-manipulation-resistant, Pareto-optimal, anonymous mechanisms for cycles of size greater than 5, and that cycles of size lower than 5 are $Z V$-line graphs. The cycle of size five is not a $Z V$-line graph, and we show the family of group-manipulation-resistant, Pareto-optimal, anonymous mechanisms for this graph, which is similar to the mechanism we present for $Z V$-line graphs.

\subsection{Related work}

Facility location problems model many common natural settings in which each agent is characterized by her ideal location and based on these locations a facility is positioned, (with some objective function in mind, e.g., locating bus stops to minimize the distance to the costumers or locating railroad stations to minimize the unpredictability of delivery schedules). The study of facility location problems arose from combinatorial optimization and aimed at finding the optimal placement of a facility or facilities to minimize transportation costs for servicing customers. Besides locating actual facilities, the problem has also found a wide range of applications in other fields such as healthcare and clustering. It is also used for modeling more abstract scenarios, not of a geographical nature, like elections (both for single winners and for committees). Because of its practical importance, variations of facility location problems have attracted significant attention for a long time from different fields, such as operations research, theoretical computer science, economics, and computational social choice.

The study of facility location problems when considering that the agents are strategic and might report untruthfully was first studied by Moulin [42]. In his work, Moulin [42] characterized the strategy-proof facility location mechanisms for the continuous line when the preferences of the agents can be represented using a single-peaked function of the facility location. Border and Jordan [6] characterized the strategy-proof facility location mechanisms for the continuous line when the agents' preferences are derived by the Euclidean distance, and Schummer and Vohra [60] considered the cases of the continuous cycle and trees. Problems of facility location on discrete graphs were also studied by Dokow et al. [18], who characterized the strategy-proof mechanisms for discrete lines and discrete cycles.

Other variants of the facility location problem were also considered in the literature. Church and Garfinkel [11], Ibara and Nagamochi [34], Cheng et al. [10] studied facets of the obnoxious facility location problem (also called the locating a bad problem or singledipped preferences). In this settings, the preference of an agent is to position the facility as far as possible from her ideal location, e.g., when positing a central polluting or noisy common facility; Feigenbaum and Sethuraman [22]; Zou and Li [82] analyzed scenarios 
in which the facility might be beneficial for some of the agents but obnoxious to others; Procaccia and Tennenholtz [53] studied scenarios in which an agent controls multiple locations; Feldman et al. [25] studied the impact of constraining the input language of the agents; and Wada et al. [73] studied a dynamic version of the problem, when agents join and leave over time and there is a cost of moving the facility, and a dynamic population version when the number of agents is not a-priori known to the designer.

The problem of positioning several facilities was also studied extensively. E.g., when the preference of an agent is defined by the distance facility closest to her [8, 20, 28, 29, $39,53]$, farthest from her [8], the average distance to a facility [61], or some (individual) convex combination of the distances [27].

For a more extensive survey on facility location problems in general, we refer the reader to the book of Farahani and Hekmatfar [21]. For a more recent survey, especially of the computational aspects of the strategic questions, we refer the interested reader to the work of Chan et al. [7].

False-name-proofness was first introduced by Yokoo et al. [79, (based on a series of previous conference papers)] in the framework of combinatorial auctions. In this work, the authors showed that the VCG mechanism does not satisfy false-name-proofness in the general case, and they proposed a property of the preferences under which this mechanism becomes false-name-proof. A similar concept was also studied in the framework of peer-topeer systems by Douceur [19] under the name Sybil attacks.

Following this work, a series of false-name-proof for various specific scenarios had been developed: Yokoo et al. [78], Yokoo [76] and Zhao et al. [81] presented mechanisms for combinatorial auctions which are false-name-proof under additional constraints on the valuations; Tsuruta et al. [70] studies redistributed mechanisms for single-item auctions; Yokoo et al. [77], Iwasaki et al. [35], and Terada and Yokoo [64] presented mechanisms for multi-unit auctions; Sakurai and Yokoo [56], Sakurai and Yokoo [57] and Yokoo et al. [80] presented mechanisms for double auction scenarios; and Suyama and Yokoo [63] studied combinatorial multi-attribute procurement auctions. For general combinatorial auctions, Yokoo et al. [78] and later Yokoo et al. [78], Lehmann et al. [38] conditions on the valuation guaranteeing that false-name bidding is not profitable in the VCG mechanism, and Todo et al. [66] showed that sub-additivity of the allocation rules characterizes the falsename-proof mechanisms.

Similar false-name-manipulation concepts were defined and analyzed for other scenarios as well. Conitzer [15] analyzed false-name-proof mechanisms in voting scenarios; Moulin [45] studied a related problem of routing-proofness in networks; Penna et al. [52] studied cost-sharing games; Todo et al. [68] studied mechanisms for online auction mechanisms, in which bidders arrive and depart over time; Todo and Conitzer [65] studied matching mechanisms; and Tsuruta et al. [71] studied false-name-proof cake-cutting procedures. A similar concept was also applied for cooperative games by Aziz et al. [4] and Lasisi and Allan [37]. In these works, they analyzed, given a weighted voting scenario, by how much an agent can change her power (as measured by the Shapley-Shubik index or the Banzhaf index), by splitting her weight among several false-name identities.

Last, in addition to the design of false-name-proof mechanisms, more direct ways to fight false-name-manipulations were also considered in the literature. For instance, adding a cost to sending too many false-name ballots [74, 75] (e.g., using CAPTCHA), attempting to create tests that are easy for a person to pass once but difficult to pass more than once [14], or adding a weak-verification procedure of some the identities (e.g., limiting the number of submissions per IP address or verifying identity uniqueness for a small number 
of agent groups [13]). ${ }^{8}$ For a short survey of this literature, we refer the interested reader to the work of Conitzer and Yokoo [16].

False-name-proof mechanisms for facility location scenarios were first studied by Todo et al. [67]. In this work, the authors characterized the false-name-proof mechanisms for facility location on the continuous line and on continuous trees. This work is the closest to ours in the false-name-proof mechanism design for facility location literature (In fact, this is the only work we are aware of that precedes the AAMAS version of our work [49]). The proof techniques we use here are different from the techniques used by Todo et al. [67]. In their work, they essentially note that any manipulation-resistant mechanism is in particular strategy-proof and using this insight to reduce the characterization problem to previous characterizations of strategy-proof mechanisms. In our work, we do not use previous characterizations but prove the properties directly.

The problem of positioning an obnoxious facility on a graph was studied by Ono et al. [51] and Todo et al. [69]. In their work, Ono et al. [51] also defined the property of rename-proofness (which is a weakening of false-name-proofness but still a generalization of strategy-proofness), and studied this property for both deterministic and random mechanisms. False-name-proof mechanisms for positioning several facilities (under heterogeneous preferences) were also studied by Sonoda et al. [62] and Ono et al. [51].

An interesting recent addition to this literature was presented by Okada et al. [50]. In this work, the authors apply automated mechanism design [58] techniques to show existence and non-existence of false-name-proof mechanisms for facility location for several graphs by translating the question to the question of formula satisfiability and using a Boolean satisfiability (SAT) solver.

\subsubsection{Approximate mechanism design}

The characterization of manipulation-resistant mechanisms for facility location is highly related to problems in Approximate mechanism design without money [53]. In these problems, agents are characterized using cardinal utilities, and the designer seeks to find an outcome maximizing a desired target function (e.g., the sum of utilities, the product of utilities, or the minimal utility). These works bound the trade-off between the target function and manipulation-resistance. They bound the loss to the target function due to manipulation-resistance constraints.

For instance, bounds on the sum of costs (the Harsanyi social welfare) were derived for false-name-proof facility location mechanisms on the continuous line and continuous trees by Todo et al. [67], strategy-proof facility location on the continuous cycle by Alon et al. [3], and for strategy-proof facility location on the discrete cycle by Dokow et al. [18]. Alon et al. [2, 3], Fotakis and Tzamos [28], and Schummer and Vohra [60] proved bounds on the maximum cost of an agent due to requiring strategyproofness, Feldman and Wilf [24] bounded the approximation of the optimal $L_{2}$ norm (sum of the squared distances of the agents) due to requiring strategy-proofness, and Feigenbaum et al. [23] bounded the approximation of the optimal $L_{p}$ norm. The approximation bounds for the problem of positioning several facilities (where the cost of an agent is her distance to the closest facility) was studied by (to name a few) Fotakis and Tzamos [29], Fotakis and Tzamos [28], Lu et al. [40], and Procaccia and Tennenholtz [53].

\footnotetext{
${ }^{8}$ Of course, we could require users to submit information that would completely identify them in the real world, but in many scenarios loosing the anonymity will drive most participants away.
} 
For a more extensive survey on approximation bounds for facility location problems, we refer the interested reader to the surveys by Cheng and Zhou [9] and by Chan et al. [7].

In this work, we do not analyze the approximation implications of the characterization, and in particular, we do not assume a specific cardinal representation of the preference of agents. Yet, we claim that for most natural representations and target functions, the approximation ratio is expected to be bad. For example, recall the mechanism for biclique graphs (Fig. 1). In this mechanism, the facility might be positioned on an 'extremely' left Z-location. Moreover, the facility might be very far from the vast majority of the agents, resulting in a very bad approximation ratio for most reasonable target functions. This phenomenon is not specific to biclique graphs. For most $Z V$-line graphs, due to the false-name-proof requirement, the facility might be positioned on a location extremely far from almost all agents, resulting in a very bad approximation ratio (roughly, the number of agents times the diameter of the graph) for most reasonable target functions. As we show in Sect. 5.1, this phenomenon is not unique to facility location problems. One faces similar lower bounds on the approximability in voting scenarios, auctions, and other mechanism design problems.

Note that modeling the agents using cardinal utilities and looking for a false-name-proof mechanism maximizing, e.g., the sum of utilities (instead of Pareto-optimality), will not circumvent this problem. Roughly speaking, the false-name-proofness means that the designer must act as-if each of the agents represents the vast majority of society since an agent should not benefit even from casting a colossal number of identical ballots. Hence, a choice to benefit some agents at the expense of others (i.e., returning a non-Pareto-optimal location) will result in an unbounded loss. Thus, we claim that when one aims to construct false-nameproof mechanisms, Pareto-optimality is essentially equivalent to most social welfare target functions, so this expected bad approximation is also expected under most cardinal notions of efficiency.

\section{Model}

Consider a graph $\mathcal{G}=\langle\mathcal{V}, \mathcal{E}\rangle$ with a set of vertices $\mathcal{V}$ and a set of undirected unweighted edges $\mathcal{E} \subseteq\left(\begin{array}{l}\mathcal{V} \\ 2\end{array}\right)$. We refer to the vertices $v \in \mathcal{V}$ also as locations and use the two terms interchangeably. The distance between two locations $v, u \in \mathcal{V}$, denoted $d(v, u)$, is the length of the shortest path connecting $v$ and $u$, and the distance between a location $v \in \mathcal{V}$ and a set of locations $S \subseteq \mathcal{V}, d(v, S)$, is defined as the minimal distance between $v$ and a location in $S$. For simplicity, we assume the graph is connected, so the distances are finite, and in Sect. 7.1 discuss the extension to disconnected graphs. We define $\mathcal{B}(v, d)$, the ball of radius $d \geqslant 0$ around a location $v \in \mathcal{V}$, to be the set of locations of distance at most $d$ from $v$,

$$
\mathcal{B}(v, d)=\{u \in \mathcal{V} \mid d(v, u) \leqslant d\} .
$$

We say that two locations are neighbors if there is an edge connecting them and denote by $N(v)$ the set of neighbors of a location $v$.

An instance of the facility location problem over $\mathcal{G}$ comprises $n$ agents who are located on vertices of $\mathcal{V}$. Formally, we represent it by a location profile $\boldsymbol{x} \in \mathcal{V}^{n}$ where $x_{i}$ is the location of Agent $i$ and $n$ is the number of agents in the profile. We also use the notation $\mathcal{V}^{\star}$ for the set of all profiles of a finite number of agents, i.e., $\mathcal{V}^{\star}=\bigcup_{t \geqslant 0} \mathcal{V}^{\star}$. We use the notations $x_{C}$ for the location profile of the agents in a given coalition of agents $C$ and $x_{-C}$ for the location profile of the agents outside of the coalition $C$. In this work, we assume the 
preference of an agent is defined by her distance to the facility: An agent located on $x \in \mathcal{V}$ strictly prefers the facility being positioned on $v \in \mathcal{V}$ over it being positioned on $u \in \mathcal{V}$ if $d(x, v)<d(x, u)$. In particular, the most preferred facility location for an agent is her own location. Given an instance $\boldsymbol{x}$, we would like to position a facility on a vertex of the graph while taking into account the preferences of the agents over the locations. We also think of $\mathcal{F}$ as a voting procedure: Each agent casts a ballot with her location, and based on the ballots, $\mathcal{F}$ returns the location for the facility.

A general facility location mechanism (or shortly a mechanism) defines a location for the facility for all location profiles of all sizes (i.e., for any number of ballots). We introduce the notation $\mathcal{V}^{\star}=\bigcup_{t \geqslant 0} \mathcal{V}^{t}$ for the set of all profiles of a finite number of agents. Hence, we represent the mechanism by a function $\mathcal{F}: \mathcal{V}^{\star} \rightarrow \mathcal{V}$. We say that a mechanism is anonymous if the outcome $\mathcal{F}(\boldsymbol{x})$ does not depend on the identities of the agents, i.e., it can be defined as a function of the ballot tally, the number of ballots for each of the locations.

\subsection{Manipulation-resistance}

A strategic agent might act untruthfully if she thinks it might cause the mechanism to return a location she prefers (that is, a location closer to her). In this work, we consider the following manipulation types:

Misreport: An agent might report to the mechanism a location different from her true location.

False-name-vote: An agent might pretend to be several agents and submit several (not necessarily identical) ballots. ${ }^{9}$

Abstention: An agent might choose not to participate in the mechanism at all.

A mechanism in which no agent benefits from these manipulations, regardless of the ballots of the other agents, is said to be strategy-proof, false-name-proof, and abstentionproof, respectively. We also consider a generalization of these manipulations to manipulations of a coalition, and say a mechanism is group-manipulation-resistant if no coalition can change the outcome, by misreporting, false-name-voting, or abstaining, to a different location that they unanimously agree is no worse than the original outcome (that is, when they vote truthfully) and at least one agent in the coalition strictly prefers the new outcome over the original outcome. Note that this is a rather strong manipulation-resistance requirement. A coalition cannot find a deviation that is beneficial for one of its members without hurting one of its other members, not even one in which different agents use different types of individual deviations.

Definition 1 (Group-manipulation-resistance ${ }^{10}$ ) An anonymous mechanism $\mathcal{F}$ is groupmanipulation-resistant if there exists no coalition of agents $C \subseteq\{1, \ldots, n\}$, a profile of locations $\boldsymbol{x} \in \mathcal{V}^{n}$, and a set of ballots ${ }^{11} \boldsymbol{A} \in \mathcal{V}^{\star}$ s.t.

\footnotetext{
${ }^{9}$ A special case of false-name-voting which is considered in the literature is double-voting: Casting the same (truthful) ballot several times to increase its impact.

${ }^{10}$ For simplicity of notations, we give the formal definition for anonymous mechanisms.

${ }^{11}$ Since $\mathcal{F}$ is an anonymous mechanisms, we define the deviation $\boldsymbol{A}$ as a set of ballots ignoring identities.
} 
(i) All the agents in $C$ weakly prefer $\mathcal{F}\left(\boldsymbol{A}, \boldsymbol{x}_{-C}\right)$, that is, the outcome when the agents outside of $C$ do not change their vote and the agents of $C$ replace their ballots by $A$, over $\mathcal{F}(\boldsymbol{x})$.

(ii) At least one agent in $C$ strictly prefers $\mathcal{F}\left(\boldsymbol{A}, \boldsymbol{x}_{-C}\right)$ over $\mathcal{F}(\boldsymbol{x})$.

We note that for $C$ being a singleton, this definition coincides with resistance to misreporting for $|\boldsymbol{A}|=1$, with resistance to false-name-voting for $|\boldsymbol{A}|>1$, and with resistance to abstention for $\boldsymbol{A}=\emptyset$.

\subsubsection{The revelation principle}

One could consider more general mechanisms in which the agents vote using more abstract ballots, and define similar manipulation-resistance notions for the general framework. Applying a simple direct revelation principle [46] shows that any such general manipulation-resistant mechanism is equivalent to a manipulation-resistant mechanism in our framework: The two mechanisms implement the same mapping of the private preferences of the agents to a location for the facility, and since the above properties are defined w.r.t. the mapping they are invariant to this transformation. That is, given some general mechanism $M$ that maps abstract actions to a location for the facility and a behavior protocol $D$ that maps types of the agents (i.e., locations) to actions of $M$, if $D$ satisfies the generalized desiderata, then the direct mechanism $M \circ D$ satisfies our desiderata.

\subsection{Efficiency}

So far, we have defined the desired manipulation-resistance properties of a mechanism. On the other hand, we would also like the mechanism to respect the preferences of the agents. We would like to avoid a scenario in which, after the mechanism has been used, the agents can agree that a different location is preferable. Given a location profile $\boldsymbol{x} \in \mathcal{V}^{\star}$, the set of Pareto-optimal locations, $P O(x)$, is the set of all locations that the agents cannot agree to rule out. Formally, given a location profile $\boldsymbol{x} \in \mathcal{V}^{\star}$ and two locations $v, u \in \mathcal{V}$, we say that $u$ Pareto-dominates $v$ w.r.t. $\boldsymbol{x}$ if

(i) all agents weakly prefer $u$ over $v$ and

(ii) at least one agent strictly prefers $u$ over $v$.

We say that $v$ is Pareto-optimal w.r.t. $\boldsymbol{x}(v \in P O(\boldsymbol{x}))$ if it is not Pareto-dominated by any other location in $\mathcal{V}$. We say a mechanism is Pareto-optimal if $\mathcal{F}(\boldsymbol{x}) \in P O(\boldsymbol{x})$ for any profile of ballots (locations) $\boldsymbol{x}$.

In particular, Pareto-optimality entails unanimity, whenever all the agents unanimously vote for the same location, the mechanism outputs this location, and entails citizen sovereignty, the mechanism is onto and does not a-priori rule out any location. When all the agents unanimously vote for the same location $\ell$, any other location is Pareto-dominated by $\ell$, so $\ell$ is the unique Pareto-optimal location, and the mechanism outputs $\ell$. Citizen sovereignty is entailed since any location $\ell$ is the output for the profile in which all ballots are equal to $\ell$.

The Pareto-optimality correspondence has several interesting combinatorial properties which are out of the scope of this work. It is easy to see that if Agent $i$ 's top-choice is location $\ell$, then $\ell$ cannot be Pareto-dominated by any other location. Hence, 
Claim $1 x \subseteq P O(x)$, for any location profile $x \in \mathcal{V}^{\star}$.

A more general property is the following.

Claim 2 Given a location profile $\boldsymbol{x} \in \mathcal{V}^{\star}$ and two locations $u$ and $v$ in $\boldsymbol{x}$, for any distance $d \in[0, d(u, v)]$ there exists a location $\ell \in \mathcal{V}$ s.t.

$$
\ell \in P O(x) \text { and }\left\{\begin{array}{l}
d(\ell, u)=d \\
d(\ell, v)=d(u, v)-d,
\end{array}\right.
$$

i.e., $\ell$ is a Pareto-optimal location that lies on a shortest path between $u$ and $v$.

Proof of Claim 2 Let $\ell^{\prime}$ be a location on some shortest path between $u$ to $v$ s.t. $d\left(\ell^{\prime}, u\right)=d$ and $d\left(\ell^{\prime}, v\right)=d(u, v)-d$. If $\ell^{\prime} \notin P O(\boldsymbol{x})$, then (since Pareto-dominance is an order) there exists a Pareto-optimal location $\ell \in P O(x)$ that Pareto-dominates $\ell^{\prime}$, and in particular

$$
d(\ell, u) \leqslant d\left(\ell^{\prime}, u\right) \quad \text { and } \quad d(\ell, v) \leqslant d\left(\ell^{\prime}, v\right) .
$$

On the other hand,

$$
d(\ell, u)+d(\ell, v) \geqslant d(u, v)=d\left(\ell^{\prime}, u\right)+d\left(\ell^{\prime}, v\right) .
$$

Hence,

$$
\left\{\begin{array}{l}
d(\ell, u)=d\left(\ell^{\prime}, u\right)=d \\
d(\ell, v)=d\left(\ell^{\prime}, v\right)=d(u, v)-d
\end{array}\right.
$$

\subsection{Relaxing false-name-proofness}

The false-name-proofness property might seem too strong desideratum since we do not bound the number of false-name-ballots a manipulator might cast. Addressing this concern, we show that assuming either abstention-proofness or strategy-proofness, this property is equivalent to resistance to only one additional false-name-ballot of the manipulator.

Definition 2 (Weak false-name-proofness) We say a mechanism $\mathcal{F}$ is a weakly-falsename-proof mechanism, if for any Agent $i$ there no profile $\boldsymbol{x}$ in which Agent $i$ can get an outcome she strictly prefers over $\mathcal{F}(\boldsymbol{x})$ by casting one additional ballot.

Proposition 1 Let $\mathcal{F}$ be an anonymous weakly-false-name-proof mechanism.

- If $\mathcal{F}$ satisfies abstention-proofness, then $\mathcal{F}$ satisfies false-name-proofness.

- If $\mathcal{F}$ satisfies strategy-proofness, then $\mathcal{F}$ satisfies false-name-proofness.

Proof Let $\boldsymbol{x} \in \mathcal{V}^{\star}$ be a profile and $A=\left\{a_{1}, \ldots, a_{k}\right\} \subseteq \mathcal{V}$ a multi-set of ballots and we will show that Agent $i$ weakly prefers the outcome $\mathcal{F}\left(x_{i}, \boldsymbol{x}_{-i}\right)$ over the outcome $\mathcal{F}\left(A, \boldsymbol{x}_{-i}\right)$, i.e., $A$ is not a false-name manipulation for Agent $i$ in $\boldsymbol{x}$. We define the following sequence of $(k+2)$ profiles: 


$$
\begin{aligned}
& -\boldsymbol{x}^{(0)}=\left\langle x_{i}, \boldsymbol{x}_{-i}\right\rangle \\
& - \text { For } t=1, \ldots, k: \boldsymbol{x}^{(t)}=\left\langle x_{i}, a_{1}, \ldots, a_{t}, \boldsymbol{x}_{-i}\right\rangle \\
& -\boldsymbol{x}^{(k+1)}=\left\langle a_{1}, \ldots, a_{k}, \boldsymbol{x}_{-i}\right\rangle=\left\langle A, \boldsymbol{x}_{-i}\right\rangle
\end{aligned}
$$

We assumed that the mechanism is resistant to Agent $i$ casting one additional ballot. Hence, Agent $i$ weakly prefers the outcome $\mathcal{F}\left(x^{(t-1)}\right)$ over the outcome $\mathcal{F}\left(\boldsymbol{x}^{(t)}\right)$ for $t=0, \ldots, k-1$. In particular, Agent $i$ weakly prefers $\mathcal{F}\left(x_{i}, \boldsymbol{x}_{-i}\right)$ over $\mathcal{F}\left(\boldsymbol{x}^{(k-1)}\right)$ and over $\mathcal{F}\left(\boldsymbol{x}^{(k)}\right)$. Now,

- If $\mathcal{F}$ satisfies abstention-proofness, then Agent $i$ weakly prefers the outcome $\mathcal{F}\left(\boldsymbol{x}^{(k)}\right)$ over the outcome $\mathcal{F}\left(\boldsymbol{x}^{(k+1)}\right)$ and hence also $\mathcal{F}\left(x_{i}, \boldsymbol{x}_{-i}\right)$ over the outcome $\mathcal{F}\left(A, x_{-i}\right)$.

- If $\mathcal{F}$ satisfies strategy-proofness, then Agent $i$ weakly prefers the outcome $\mathcal{F}\left(\boldsymbol{x}^{(k-1)}\right)$ over the outcome $\mathcal{F}\left(\boldsymbol{x}^{(k+1)}\right)$ and hence also $\mathcal{F}\left(x_{i}, \boldsymbol{x}_{-i}\right)$ over the outcome $\mathcal{F}\left(A, x_{-i}\right)$.

\subsection{Order-based mechanisms}

A natural class of Pareto-optimal anonymous mechanisms are the Order-based mechanisms. These mechanisms are defined using an order $\pi$ over the locations. Given a location profile $\boldsymbol{x} \in \mathcal{V}^{\star}$ the mechanism returns the first location according to $\pi$ in $P O(\boldsymbol{x})$. It is easy to see that these mechanisms are Pareto-optimal, anonymous, and invariant to agents casting the same ballot several times, i.e., these mechanisms depend of the support of $\boldsymbol{x}$. In many scenarios, it is easier to analyze which properties of the order $\pi$ will result in manipulation resistance of the mechanism than analyzing the full class of mechanisms.

In Appendix A, we show that the anonymous, Pareto-optimal, group-manipulationresistant, order-based mechanisms are a strict subset of the anonymous, Pareto-optimal, group-manipulation-resistant mechanisms, and that there might be anonymous Paretooptimal mechanisms which are not order-based mechanisms and even group-manipulation-resistant mechanisms that are not invariant to ballot-duplication. This, in contrast to the general voting scenario when the preferences are unconstrained. For this scenario, Conitzer [15, Corollary 1] showed that for any false-name-proof voting rule, given that a vote is cast at least once, it does not matter how many times it is cast.

Claim 3 There exists an anonymous, Pareto-optimal, group-manipulation-resistant mechanism $\mathcal{F}$ for the $(2,3)$-biclique s.t. $\mathcal{F}$ is not an order-based mechanism.

Claim 4 There exists an anonymous, Pareto-optimal, group-manipulation-resistant mechanism $\mathcal{F}$ for the cycle of size four s.t. $\mathcal{F}$ is not invariant to ballot duplication. In particular, $\mathcal{F}(\boldsymbol{x})$ cannot be defined as a function of the support of $\boldsymbol{x}$. 


\section{Simple $Z V$-line graphs}

In this work, we define a family of graphs, $Z V$-line graphs, and present a simple and general mechanism for this family. This family is defined by introducing a simple combinatorial structure: A partition of the locations into two categories and a connectivity constraint. One could think of the partition as representing a social agreement or a social norm according to which the mechanism is defined, e.g., a subset of status-quo locations or an a-priori priority hierarchy over the locations. The connectivity constraint (as the graph in general) represents homogeneity over the preferences of different agents, that is, a restriction over the possible preference profiles, focusing on scenarios where the preferences share some common structure. This allows us to construct a group-manipulation-resistant mechanism.

We first define the basic case, the simple $Z V$-line graphs family, and afterwards extend it recursively to define general $Z V$-line graphs. ${ }^{12}$

Definition 3 (Simple $Z V$-line graphs) An unweighted undirected connected graph $\mathcal{G}=\langle\mathcal{V}, \mathcal{E}\rangle$ is a simple $Z V$-line graph w.r.t. a partition of the vertices into two disjoint sets $\mathcal{V}=V \bigcup Z$ and a full linear order over $Z$, if

- There are no edges between different locations in $V$ ( $V$-locations), i.e., for any $V$ -location $v \in V, N(v) \subseteq Z$.

Moreover, for any $V$-location $v \in V, N(v)$ is a contiguous sequence of $Z$-locations.

- For any $Z$-location $z \in Z, \mathcal{B}(z, 1) \cap Z$ is a contiguous sequence of $Z$-locations.

We say that a graph $\mathcal{G}=\langle\mathcal{V}, \mathcal{E}\rangle$ is a simple $Z V$-line graph, if it is a simple $Z V$-line graph w.r.t. some partition of the vertices into two disjoint sets $\mathcal{V}=V \bigcup \dot{U} Z$ and a full linear order over $Z$.

For simplicity of description, we refer later to the order over the $Z$-locations as a left-toright order. We also use the terms $V$-locations, $Z$-locations, $V$-vertices, and $Z$-vertices for the respective sets of locations.

For example, the graphs of Figs. 1, 2, 3, 5 and 6 in the introduction are all simple $Z V$ -line graphs:

- There are no edges between $Z$-locations, so $\mathcal{B}(z, 1) \cap Z=\{z\}$ for all $z \in Z$, and they are ordered on a horizontal line.

- The $V$-locations are the vertices denoted by

- Each $V$-location is connected to a contiguous sequence of $Z$-locations.

Two examples of simple $Z V$-line graphs with no $V$-locations are the clique graph with any order over the locations and the line graph with the natural order (note this is a different representation as a simple $Z V$-line graph from the one in Fig. 2). Note also that bicliques are $Z V$-line graphs w.r.t. any order over the $Z$-locations. That is, a graph could be a simple $Z V$-line graph w.r.t. several representations.

\footnotetext{
12 In the conference version of this work [49], we presented a different but equivalent definition of $Z V$-line graphs.
} 
Given a simple $Z V$-line graph $\mathcal{G}=\langle\mathcal{V}, \mathcal{E}\rangle$ that is a simple $Z V$-line graph w.r.t. a given partition of the vertices $\mathcal{V}=V \bigcup \dot{U} Z$ and an order over $Z$, we define the following facility location mechanism $\mathcal{F}_{\text {sZV }}^{\star}: \mathcal{V}^{\star} \rightarrow \mathcal{V}$.

Definition $4\left(\mathcal{F}_{\mathrm{sZV}}^{\star}-A\right.$ mechanism for simple $Z V$-line graphs) For a simple $Z V$-line graph $\mathcal{G}=\langle\mathcal{V}, \mathcal{E}\rangle$, given a ballot profile (location reports of the agents) $x \in \mathcal{V}^{\star}$,

If all agents voted unanimously to a unique location $\ell \in \mathcal{V}$ (i.e., all ballots are identical), return $\ell$.

$\checkmark$ Otherwise, return the leftmost Pareto-optimal Z-location.

First, we claim that $\mathcal{F}_{\text {sZV }}^{\star}$ satisfies the following properties:

$\mathcal{F}_{\text {sZV }}^{\star}$ is well-defined: (I.e., there is always a Pareto-optimal $Z$-location in the second case): If one of the ballots is a $Z$-location, then this location is Pareto-optimal. In case that all ballots are $V$-locations and there exist two non-identical ballots $u$ and $v$, then by Claim 2 there exists a Pareto-optimal location $\ell$ in distance 1 of $u$. Since all neighbors of a $V$-location are $Z$-locations, we get that $\ell \in Z$.

$\mathcal{F}_{\text {sZV }}^{\star}$ can be computed in polynomial time: We can find all Pareto-dominated locations (i.e., $\mathcal{V} \backslash P O(\boldsymbol{x})$ ) in polynomial time in the following way:

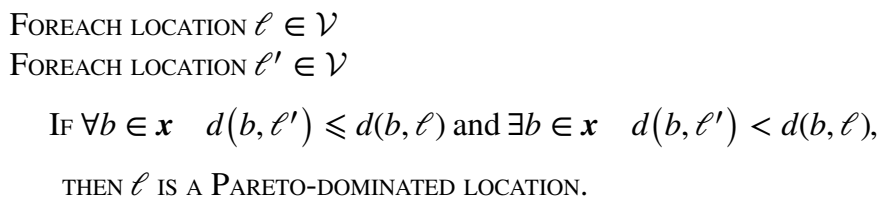

Hence, finding $P O(x)$ and finding the leftmost location in $P O(x) \cap Z$ can be done in polynomial time. Since finding whether all ballots are identical can be done in polynomial time, we get that $\mathcal{F}_{\text {sZV }}^{\star}$ can be computed in polynomial time.

$\mathcal{F}_{\text {sZV }}^{\star}$ is an order-based mechanism: $\mathcal{F}_{\text {sZV }}^{\star}$ can be equivalently defined as returning the first Pareto-optimal location in $\mathcal{V}$ according to the following (predefined) order: First, check whether any of the $Z$-locations is a Pareto-optimal location, from left to right; Then, iterate over the $V$-locations in some arbitrary order. This is an equivalent definition since there is no Pareto-optimal $Z$-location only in case that all ballots are identical.

Note that $\mathcal{F}_{\text {sZV }}^{\star}$ is defined w.r.t. a given partition and order, so when there are several different partitions for $\mathcal{V}$, e.g., when $\mathcal{G}$ is a biclique, different mechanisms could arise. It is also important to note that we do not assume that the agents know the partition and the order, but they know the mechanism $\mathcal{F}_{\text {sZV }}^{\star}$. In other words, we see this structure as a combinatorial property of a graph that derives the preferences of agents and could represent some homogeneity of the preferences or a social norm of giving priority to the $Z$-locations.

Proposition $2\left(\mathcal{F}_{\mathrm{sZV}}^{\star}\right.$ satisfies the desired properties) Let $\mathcal{G}=\langle\mathcal{V}, \mathcal{E}\rangle$ be a simple $Z V$-line graph. Then $\mathcal{F}_{\mathrm{sZV}}^{\star}$ is an anonymous, Pareto-optimal, group-manipulation-resistant mechanism for $\mathcal{G}$. That is, $\mathcal{F}_{\mathrm{sZV}}^{\star}$ satisfies: 
For any location profile $\boldsymbol{x} \in \mathcal{V}^{\star}$, a coalition of agents $C$, and a set of ballots $\boldsymbol{A} \in \mathcal{V}^{\star}, \boldsymbol{A}$ is not a beneficial deviation for $C .^{13}$

Proof of Proposition 2 The anonymity of $\mathcal{F}_{\mathrm{sZV}}^{\star}$ is trivial from its definition as a function of the ballots while ignoring the identity of the agents. It is also easy to see that the outcome of $\mathcal{F}_{\text {sZV }}^{\star}$ will always be a Pareto-optimal location. Both in the first case, in which it equals the set of all ballots, and in the second case, in which it is a Pareto-optimal Z-location.

To prove the main part of the proposition, we assume towards a contradiction that there exists a profile of locations (ballots) $\boldsymbol{x} \in \mathcal{V}^{\star}$, a coalition of agents $C$, and a set of ballots $\boldsymbol{A} \in \mathcal{V}^{\star}$, s.t. the coalition $C$ can cast $\boldsymbol{A}$ and change the outcome to be $\mathcal{F}_{\text {sZV }}^{\star}\left(\boldsymbol{A}, \boldsymbol{x}_{-C}\right)$ that it strictly prefers. That is, all the agents in $C$ weakly prefer $\mathcal{F}_{\mathrm{sZV}}^{\star}\left(\boldsymbol{A}, \boldsymbol{x}_{-C}\right)$ over $\mathcal{F}_{\mathrm{sZV}}^{\star}(\boldsymbol{x})=\mathcal{F}_{\mathrm{sZV}}^{\star}\left(\boldsymbol{x}_{C}, \boldsymbol{x}_{-C}\right)$ and at least one agent in $C$, Agent $i$ for $i \in C$, strictly prefers $\mathcal{F}_{\text {sZV }}^{\star}\left(\boldsymbol{A}, \boldsymbol{x}_{-C}\right)$ over $\mathcal{F}_{\text {sZV }}^{\star}(\boldsymbol{x}) . \mathcal{F}_{\text {sZV }}^{\star}(\boldsymbol{x}) \in P O(\boldsymbol{x})$ and in particular the coalition of all agents does not strictly prefer $\mathcal{F}_{\mathrm{sZV}}^{\star}\left(\boldsymbol{A}, \boldsymbol{x}_{-C}\right)$ over $\mathcal{F}_{\mathrm{sZV}}^{\star}(\boldsymbol{x})$. Hence, there exists an Agent $j$, for $j \notin C$, who strictly prefers $\mathcal{F}_{\text {sZV }}^{\star}(\boldsymbol{x})$ over $\mathcal{F}_{\text {sZV }}^{\star}\left(\boldsymbol{A}, \boldsymbol{x}_{-C}\right)$.

If $\mathcal{F}_{\text {sZV }}^{\star}(\boldsymbol{x})$ is a $V$-location: Then necessarily, all the ballots in $\boldsymbol{x}$ are identical and equal

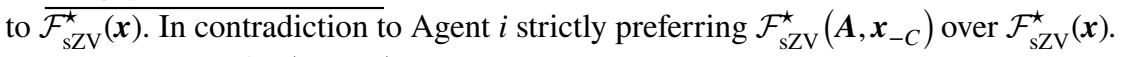

Similarly, if $\mathcal{F}_{\mathrm{sZV}}^{\star}\left(\boldsymbol{A}, \boldsymbol{x}_{-C}\right)$ is a $V$-location: Then necessarily, all the ballots in $\left\langle\boldsymbol{A}, \boldsymbol{x}_{-C}\right\rangle$ are identical and equal to $\mathcal{F}_{\text {sZV }}^{\star}\left(\boldsymbol{A}, \boldsymbol{x}_{-C}\right)$. In contradiction to Agent $j$ strictly preferring $\mathcal{F}_{\text {sZV }}^{\star}(\boldsymbol{x})$ over $\mathcal{F}_{\text {sZV }}^{\star}\left(\boldsymbol{A}, \boldsymbol{x}_{-C}\right)$.

If both $\mathcal{F}_{\text {sZV }}^{\star}(\boldsymbol{x})$ and $\mathcal{F}_{\text {sZV }}^{\star}\left(\boldsymbol{A}, \boldsymbol{x}_{-C}\right)$ are $Z$-locations:

First, we prove the following two auxiliary lemmata.

Lemma (i) For any $v \in \mathcal{V}$ and $d \geqslant 1, \mathcal{B}(v, d) \cap Z$ is a non-empty contiguous sequence of Z-locations.

Proof We prove the lemma via induction over $d$.

For $d=1: \mathcal{B}(v, 1) \cap Z$ is a contiguous sequence of $Z$-locations by the definition of simple $Z V$-line graphs.

For $d \geqslant 2: \mathcal{B}(v, d) \cap Z=\bigcup_{u \in \mathcal{B}(v, 1)}(\mathcal{B}(u, d-1) \cap Z)$. Next, by noticing that for any $u \in \mathcal{B}(v, 1) \backslash\{v\}$

- By the induction hypothesis $\mathcal{B}(u, d-1) \cap Z$ is a non-empty contiguous sequence of Z-locations;

- $\quad(\mathcal{B}(u, 1) \cap Z) \bigcap(\mathcal{B}(v, 1) \cap Z) \neq \emptyset$, since either $u$ or $v$ is a $Z$-vertex in the intersection; and

- $(\mathcal{B}(u, d-1) \cap Z) \bigcap(\mathcal{B}(v, d-1) \cap Z) \supseteq(\mathcal{B}(u, 1) \cap Z) \bigcap(\mathcal{B}(v, 1) \cap Z)$,

we set that all the operands in $\bigcup_{u \in \mathcal{B}(v, 1)}(\mathcal{B}(u, d-1) \cap Z)$ intersect the non-empty contiguous sequence of $Z$-locations $\mathcal{B}(v, d-1) \cap Z$, and hence also $\mathcal{B}(v, d) \cap Z$ is a non-empty contiguous sequence of $Z$-locations.

\footnotetext{
${ }^{13}$ Since $\mathcal{F}_{\text {sZV }}^{\star}$ is onto, this property entails Pareto-optimality. Yet, we prefer to state explicitly Pareto-optimality as a desired efficiency property.
} 
Lemma (ii) Let $\boldsymbol{x}$ be a location profile s.t. $\mathcal{F}_{\mathrm{sZV}}^{\star}(\boldsymbol{x}) \in Z$ and let $v \in Z$ be a location s.t. Agent $i$ strictly prefers $v$ over $\mathcal{F}_{\mathrm{sZV}}^{\star}(\boldsymbol{x})$. Then $\mathcal{F}_{\mathrm{sZV}}^{\star}(\boldsymbol{x})$ is to the left of $v$.

Proof If $d\left(x_{i}, v\right)=0$, i.e., $x_{i}=v$, then it is a $Z$-location so $v=x_{i} \in P O(\boldsymbol{x}) \cap Z$. By the definition of $\mathcal{F}_{\text {sZV }}^{\star}, \mathcal{F}_{\text {sZV }}^{\star}(\boldsymbol{x})$ is to the left of $v$. Henceforth, we assume that $d\left(x_{i}, v\right) \geqslant 1$.

If $x_{i}$ is a $Z$-location, then $x_{i} \in P O(x) \cap Z$ and by the definition of $\mathcal{F}_{\mathrm{sZV}}^{\star}, \mathcal{F}_{\mathrm{sZV}}^{\star}(\boldsymbol{x})$ is to the left of $x_{i}$. Since $\mathcal{F}_{\text {sZV }}^{\star}(\boldsymbol{x}) \notin \mathcal{B}\left(x_{i}, d\left(x_{i}, v\right)\right) \cap Z$ and since by Lemma (i), $\mathcal{B}\left(x_{i}, d\left(x_{i}, v\right)\right) \cap Z$ is a non-empty contiguous sequence of $Z$-locations which includes $x_{i}$, we get that $\mathcal{F}_{\mathrm{sZV}}^{\star}(\boldsymbol{x})$ is to the left of $\mathcal{B}\left(x_{i}, d\left(x_{i}, v\right)\right) \cap Z$ and in particular to the left of $v$.

If $x_{i}$ is a $V$-location, then since $\mathcal{F}_{\text {sZV }}^{\star}(\boldsymbol{x}) \neq x_{i}$ there exists an Agent $j$ s.t. $x_{j} \neq x_{i}$ and by Claim 2 there exists a location $\ell \in P O(x)$ s.t. $d\left(\ell, x_{i}\right)=1$, i.e., $\ell \in N\left(x_{i}\right)$. By the definition of simple $Z V$-line graphs, $\ell$ is a $Z$-location and hence $\mathcal{F}_{\mathrm{sZV}}^{\star}(\boldsymbol{x})$ is to the left of $\ell$ (or is equal to it). Since by Lemma (i),

$$
\ell \in \mathcal{B}\left(x_{i}, 1\right) \cap Z \subseteq \mathcal{B}\left(x_{i}, d\left(x_{i}, v\right)\right) \cap Z,
$$

both sets are contiguous sequences of $Z$-locations, and $\mathcal{F}_{\text {sZV }}^{\star}(\boldsymbol{x}) \notin \mathcal{B}\left(x_{i}, d\left(x_{i}, v\right)\right) \cap Z$, we get that $\mathcal{F}_{\mathrm{sZV}}^{\star}(\boldsymbol{x})$ is to the left of $v$.

By applying Lemma (ii) for the profile $\boldsymbol{x}$ and Agent $i$, we get that $\mathcal{F}_{\text {sZV }}^{\star}(\boldsymbol{x})$ is to the left of $\mathcal{F}_{\text {sZV }}^{\star}\left(\boldsymbol{A}, \boldsymbol{x}_{-C}\right)$. Similarly, by applying this lemma for the profile $\left(\boldsymbol{A}, \boldsymbol{x}_{-C}\right)$ and Agent $j$, we get that $\mathcal{F}_{\text {sZV }}^{\star}\left(\boldsymbol{A}, \boldsymbol{x}_{-C}\right)$ is to the left of $\mathcal{F}_{\text {sZV }}^{\star}(\boldsymbol{x})$. Hence, we get a contradiction.

Note that bipartiteness of the graph is not a sufficient condition for group-manipulationresistance of $\mathcal{F}_{\mathrm{sZV}}^{\star}$. For example, $C_{6}$, the cycle of size 6 , is a bipartite graph but there exists no anonymous Pareto-optimal mechanism for $C_{6}$ which is resistant even to manipulations by a single agent (In Sect. 6, we generalize this and show that there exists no such mechanism for $C_{n}$ for any $n \geqslant 6$ ).

Proposition 3 There is no anonymous Pareto-optimal mechanism for $C_{6}$, the cycle of six locations, that is resistant even to manipulations of a single agent.

Proof Assume towards a contradiction that $\mathcal{F}$ is a Pareto-optimal, anonymous, groupmanipulation-resistant mechanism for $C_{6}$. We denote the locations of $C_{6}$ by $\{0,1,2,3,4,5\}$, and w.l.o.g. assume that for the profile of six agents who vote for all six locations the outcome is 0 (see Fig. 8).

For the profile $\langle\mathbf{2}, \mathbf{4}, \mathbf{5}\rangle$ : From resistance to false-name manipulations of the first and last agents, the outcome must be either 0 or 4 (Since any of them can change the result to

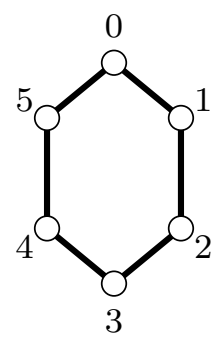

Fig. 8 The cycle of size $6, C_{6}$ 


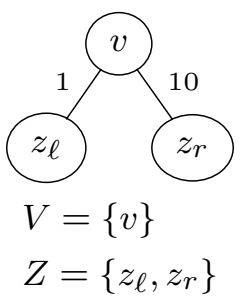

Fig. 9 Proposition 2 does not hold for weighted graphs

be 0 by adding false-ballots). From the Pareto-optimality of $\mathcal{F}$, the outcome cannot be 0 which is Pareto-dominated by 4 . Hence, the outcome for the profile $\langle\mathbf{2}, \mathbf{4 ,} \mathbf{5}\rangle$ is 4 .

Similarly, for the symmetric profile $\langle\mathbf{1}, \mathbf{2}, \mathbf{4}\rangle$ the outcome must be 2 . From false-nameresistance the outcome for the profile $\langle\mathbf{2}, \mathbf{4}\rangle$ must also be 2 (Otherwise, the first agent will cast an additional false-ballot 1 to get the outcome to be 2 ).

But, the second agent in the profile $\langle\mathbf{2}, \mathbf{4}\rangle$ (who is located on 4) can change the outcome to be 4 that she strictly prefers by casting one additional false-name ballot 5 . So we get a contradiction.

We also note that Proposition 2 does not hold for weighted graphs. Consider the weighted graph in Fig. 9 and a profile in which Alice is located on $z_{r}$ and Bob is located on $v$. Then the outcome of $\mathcal{F}_{\mathrm{sZV}}^{\star}$ is $z_{r}$, but Bob can move the facility to a preferred location $z_{\ell}$ both (i) by misreporting $z_{\ell}$; hence, $\mathcal{F}^{\star}$ is not strategy-proof; and (ii) by false-name-voting $z_{\ell}$ besides his truthful ballot, hence, $\mathcal{F}_{\text {sZV }}^{\star}$ is not false-name-proof. ${ }^{14}$

Last, we note that there exist simple mechanisms that satisfy subsets of the properties of Proposition 2:

- The fixed mechanism, which always positions the facility on a predefined location ignoring the votes of the agents, is trivially group-manipulation-resistant and anonymous for any graph, but it is not onto and hence not Pareto-optimal.

- A dictatorship of the first agent, i.e., a mechanism that always positions the facility on the location reported by the first agent, is not anonymous, but it is group-manipulation-resistant for any graph. ${ }^{15}$

- The median mechanism, which minimizes the sum of distances between the facility and the ballots, is an anonymous Pareto-optimal mechanism for any graph. For some graphs, e.g., the discrete line, it also satisfies strategy-proofness and abstention-proofness both against one manipulator and against a coalition, but an agent can benefit by casting multiple identical ballots.

- Also the mean mechanism, which minimizes the sum of squares of the distances between the facility and the ballots, is an anonymous Pareto-optimal mechanism for any graph, but it might not be strategy-proof or false-name-proof even against one agent, e.g., for the discrete line graph (it is abstention-proof, though).

\footnotetext{
${ }^{14}$ The mechanism that returns the leftmost ballot according to the order $z_{\ell}-v-z_{r}$ satisfies the desiderata. Notice that this mechanism can be defined as simple $Z V$-line graph w.r.t. a different partition: $Z=\left\{z_{\ell}, v, z_{r}\right\}$ and $V=\emptyset$.

${ }^{15}$ While we did not formally define false-name-proofness for non-anonymous mechanisms, assuming a false-name-ballot cannot be counted as the vote of the first agent, no agent can benefit from casting additional ballots.
} 


\subsection{Examples of Simple ZV-line graphs}

In this subsection, we study several graph families that we show are simple $Z V$-line graphs, by that also illustrating the richness of the family of simple $Z V$-line graphs. We also present some of the mechanisms for these graphs that are derived from our main result. As we saw previously, cliques, lines, and bicliques are simple $Z V$-line graphs.

Claim 5 For any $n \geqslant 1$, the clique over $n$ locations,

$$
K_{n}=\langle\mathcal{V}=\{1, \ldots n\}, \mathcal{E}=\{(i, j) \mid i \neq j\}\rangle
$$

(that is, the graph over $n$ locations with an edge between any two locations), is a simple $Z V$ -line graph w.r.t. the $Z$-locations being all $n$ locations with any order over them.

Claim 6 For any $n \geqslant 1$, the discrete line of $n$ locations,

$$
P_{n}=\langle\mathcal{V}=\{1, \ldots n\}, \mathcal{E}=\{(i, i+1) \mid i=1, \ldots, n-1\}\rangle,
$$

is a simple $Z V$-line graph w.r.t. the following partitions of $\mathcal{V}$

- w.r.t. the $Z$-locations being all $n$ locations with the natural order over them;

- w.r.t. the $Z$-locations being the $\left\lceil\frac{n}{2}\right\rceil$ odd-indexed locations with the natural order over them; or in general

- w.r.t. the $Z$-locations being the complement of an independent set of vertices.

(Note that these vertex partitions result in different mechanisms).

Claim 7 For any $n, m \geqslant 1$, the biclique over $n$ and $m$ locations,

$$
K_{m, n}=\langle\mathcal{V}=\{1, \ldots n\} \dot{\cup}\{n+1, \ldots n+m\}, \mathcal{E}=\{(i, j) \mid i \leqslant n<j\}\rangle
$$

(that is, the graph with $n$ locations on one side, $m$ locations on the other side and an edge between any two locations of opposite sides), is a simple $Z V$-line graph w.r.t. $Z$-locations being the first $n$ locations with any order over them and the other $m$ locations being the $V$ -locations.

Next, we note that all connected graph with at most five locations but one are simple $Z \mathrm{~V}$ -line graphs.

Claim 8 Let $\mathcal{G}=\langle\mathcal{V}, \mathcal{E}\rangle$ be a connected graph with at most five locations, $|\mathcal{V}| \leqslant 5$. If $\mathcal{G} \neq C_{5}$ (the cycle of size 5), then $\mathcal{G}$ is a simple $Z V$-line graph. $\left(C_{5}\right.$ is not a simple $Z V$-line graphs but still there exists a group-manipulation-resistant, anonymous, Pareto-optimal mechanism for $C_{5}$ ).

We prove this claim in Appendix B by showing for each of the graphs a partition to $V$-locations and Z-locations with an order over the latter. In Sect. 6 we show that $C_{5}$ is not a simple $Z V$-line graphs but still there exists a group-manipulation-resistant, anonymous, Pareto-optimal mechanism for $C_{5}$ which is also an order-based mechanism.

Last, the following three propositions show that small perturbations of cliques, the outcome of adding locations or removing edges, are also simple $Z V$-line graphs. 


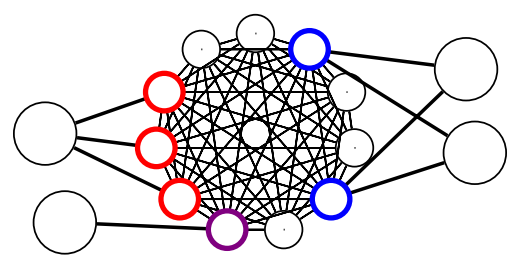

Fig. 10 Example 15 for Proposition 4 (Color figure online)

Proposition 4 Let $\mathcal{G}=\langle\mathcal{V}, \mathcal{E}\rangle$ be a graph with a vertex set $\mathcal{V}$ and an edge set $\mathcal{E}$, and let $\mathcal{V}=\tilde{V} \dot{\cup} V^{(1)} \dot{U} V^{(2)} \dot{U} \cdots \dot{U} V^{(k)}($ for $k \geqslant 1$ and $\tilde{V} \neq \emptyset)$ be a partition of the vertex set $\mathcal{V}$ s.t. (See Fig. 10)

- The restriction of $\mathcal{G}$ to $\mathcal{V} \backslash \widetilde{V}$ is a clique. That is, for any two locations $v, u \notin \widetilde{V} \quad(v, u) \in \mathcal{E} ;$ and

- For any location $v \in \widetilde{V}, N(v)=V^{(i)}$ for some partition element $V^{(i)}(i \in\{1, \ldots, k\})$

Then $\mathcal{G}$ is a simple $Z V$-line graph.

Proof We claim that $\mathcal{G}$ is a simple $Z V$-line graph w.r.t. taking the $Z$-locations to be $Z=\mathcal{V} \backslash \widetilde{V}$ and an order over $Z$ s.t. $V^{(i)}$ is a contiguous sequence of locations for $i=1, \ldots, k$. Note that such an order exists since the sets $V^{(i)}$ are disjoint.

- There are no edges between different $V$-locations.

- For any $Z$-location $z \in Z, \mathcal{B}(z, 1) \cap Z=\mathcal{V} \backslash \widetilde{V}=Z$.

- For $i=1, \ldots, k, N\left(v_{i}\right)$ is a contiguous sequence of $Z$-locations by our choice of the order.

Hence, $\mathcal{G}$ is a simple $Z V$-line graph.

Proposition 5 Let $K_{n} \backslash$ e be the outcome of removing one edge from a clique of size $n$. That is, $K_{n} \backslash e:=\langle\mathcal{V}, \mathcal{E}\rangle$ s.t. $|\mathcal{V}|=n$ and there exist two locations $u, v \in \mathcal{V}$ s.t. $\mathcal{E}=\left(\begin{array}{l}\mathcal{V} \\ 2\end{array}\right) \backslash\{(u, v)\}$. Then $\mathcal{G}$ is a simple $Z V$-line graph.

Proof W.1.o.g., assume that $\mathcal{E}=\left(\begin{array}{l}\mathcal{V} \\ 2\end{array}\right) \backslash\left\{\left(v_{1}, v_{2}\right)\right\}$. Then by choosing $\widetilde{V}=\left\{v_{1}\right\}, V^{(1)}=$ $\left\{v_{2}, \ldots, v_{n}\right\}$, and applying Proposition 4 we get that $K_{n} \backslash e$ is a simple $Z V$-line graph (w.r.t. $Z=\left\{v_{2}, \ldots, v_{n}\right\}$ and any order over $Z$ ).

Generalizing Proposition 5, we get the following proposition.

Proposition 6 For $1<m<n$, let $K_{n} \backslash K_{m}$ be the outcome of removing a clique of size $m$ from the clique of size $n$ (See Fig. 11),

$$
K_{n} \backslash K_{m}:=\langle\mathcal{V}=\{1, \ldots n\}, \mathcal{E}=\{(i, j) \mid i \neq j\} \backslash\{(i, j) \mid i, j \leqslant m \text { and } i \neq j\}\rangle .
$$

Then $\mathcal{G}$ is a simple $Z V$-line graph. 

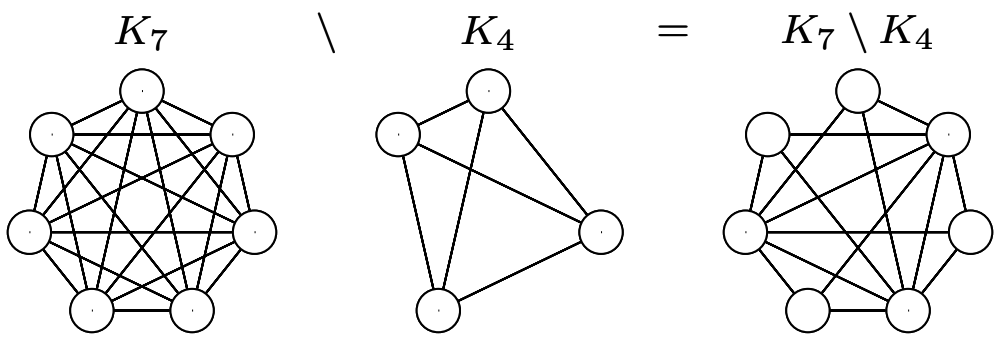

Fig. $11 K_{7} \backslash K_{4}$

Proof We define $\widetilde{V}=\left\{v_{1}, \ldots, v_{m}\right\}, V^{(1)}=\left\{v_{m+1}, \ldots, v_{n}\right\}$. We note that for any location $v \in \widetilde{V}, N(v)=V^{(1)}$ and that the graph restricted to $V^{(1)}$ is a clique of size $(n-m)$. Hence, by applying Proposition 4 we get that $K_{n} \backslash K_{m}$ is a simple $Z V$-line graph (w.r.t. $Z=\{m+1, \ldots, n\}$ and any order over $Z$ ).

\section{$4 Z V$-line graphs}

Next, we extend our result for a larger family of graphs. In this section, we show two recursive constructions, of graphs and of mechanisms, extending the desired mechanism for simple $Z V$-line graphs we showed, to a wider family which we name $Z V$-line graphs.

Given a graph $\mathcal{G}=\langle\mathcal{V}, \mathcal{E}\rangle$ we say that a sequence of non-empty sets of locations $V_{0}, V_{1}, \cdots, V_{k} \subseteq \mathcal{V}(k \geqslant 0)$ is a non-trivial cover of $\mathcal{V}$ if

$$
\begin{aligned}
& \mathcal{V}=V_{0} \cup V_{1} \cup \cdots \cup V_{k} \\
& \text { and } V_{i} \nsubseteq V_{j} \text { for all } i \neq j .
\end{aligned}
$$

We extend the definition of simple $Z V$-line graphs to define general $Z V$-line graphs using the following inductive construction. We also define one of the locations of the $Z V$-line graph to be the root of the graph, and denote it by $\mathcal{R}(\mathcal{G})$.

Definition 5 ( $Z V$-line graphs)

- Any simple $Z V$-line graph $\mathcal{G}=\langle\mathcal{V}, \mathcal{E}\rangle$ is a $Z V$-line graph, and we define its root $\mathcal{R}(\mathcal{G})$ to be its leftmost $Z$-location.

- Given an unweighted undirected connected graph $\mathcal{G}=\langle\mathcal{V}, \mathcal{E}\rangle$ and a non-trivial cover of its vertices, $V_{\perp}, V_{1}, \cdots, V_{k} \subseteq \mathcal{V}(k \geqslant 1): \mathcal{G}$ is a $Z V$-line graph w.r.t. the cover $V_{\perp}, V_{1}, \cdots, V_{k}$ if

1. The projection of $\mathcal{G}$ on $V_{\perp}, \mathcal{G}_{\perp}=\left\langle\mathcal{V}=V_{\perp}, \mathcal{E}=\mathcal{E} \cap\left(\begin{array}{c}V_{\perp} \\ 2\end{array}\right)\right\rangle$, is a $Z V$-line graph. For $i=1, \ldots, k$ :

2. The projection of $\mathcal{G}$ on $V_{i}, \mathcal{G}_{i}=\left\langle\mathcal{V}=V_{i}, \mathcal{E}=\mathcal{E} \cap\left(\begin{array}{c}V_{i} \\ 2\end{array}\right)\right\rangle$, is a $Z V$-line graph.

3. The root of $\mathcal{G}_{i}, \mathcal{R}\left(\mathcal{G}_{i}\right)$, is the unique location in $V_{i} \cap V_{\perp}$. 
4. There are no edges between locations in $\left(V_{i} \backslash\left\{\mathcal{R}\left(\mathcal{G}_{i}\right)\right\}\right)$ and locations outside of $V_{i}$. (Equivalently, all paths between locations of $V_{i}$ and locations outside of $V_{i}$ include the root $\mathcal{R}\left(\mathcal{G}_{i}\right)$ ).

5. $\left(V_{i} \backslash\left\{\mathcal{R}\left(\mathcal{G}_{i}\right)\right\}\right) \cap\left(V_{j} \backslash\left\{\mathcal{R}\left(\mathcal{G}_{j}\right)\right\}\right)=\emptyset \quad$ for $i \neq j$ in $\{1, \ldots, k\}$.

If $\mathcal{G}$ is a $Z V$-line graph w.r.t. the cover $V_{\perp}, V_{1}, \cdots, V_{k}$, we define its root to be $\mathcal{R}(\mathcal{G})=\mathcal{R}\left(\mathcal{G}_{\perp}\right)$.

We say that a graph $\mathcal{G}=\langle\mathcal{V}, \mathcal{E}\rangle$ is a $Z V$-line graph, if it is either a simple $Z V$-line graph or a $Z V$-line graph w.r.t. some non-trivial cover of $\mathcal{V}$.

For ease of description, we refer to the inductive operation of connecting a graph $\mathcal{G}_{i}$ to $\mathcal{G}_{\perp}$ as above as planting. We extend the root function $\mathcal{R}(\cdot)$ to locations and sets of locations of non-simple $Z V$-line graphs. Given a $Z V$-line graph $\mathcal{G}$ w.r.t. a cover $V_{\perp}, V_{1}, \cdots, V_{k}$, we define the root of a location $v \in \mathcal{V}, \mathcal{R}(v)$, to be $v$ if $v \in V_{\perp}$ and $\mathcal{R}\left(\mathcal{G}_{i}\right)$ if $v_{i} \in V_{i} \backslash V_{\perp}$ for some $i \in\{1, \ldots, k\}$. Given a set (or a profile) of locations we define the root of the set to be $\mathcal{R}(S)=\{\mathcal{R}(v) \mid v \in S\}$, i.e., the roots of the locations in $S$.

For example, Fig. 7 in the introduction can be constructed in three stages in the following way (see Fig. 12).

Figure $12 \mathrm{a}$ is a simple $Z V$-line graph as we saw in Fig. 3.

Figure $12 \mathrm{~b}$ is the outcome of 'planting' a 6-Clique $\left(\mathcal{G}_{1}\right)$, a $(4,4)$-biclique $\left(\mathcal{G}_{2}\right)$, a 9-Clique $\left(\mathcal{G}_{3}\right)$, a line $\left(\mathcal{G}_{4}\right)$, a 2-clique $\left(\mathcal{G}_{5}\right)$, a $(1,3)$-biclique $\left(\mathcal{G}_{6}\right)$, and a 2 -Clique $\left(\mathcal{G}_{7}\right)$ on locations of Fig. 12a and hence it is a $Z V$-line graph.

$\checkmark$ Figure $12 \mathrm{c}$ is the outcome of 'planting' a $(1,3)$-biclique $\left(\mathcal{G}_{8}\right)$ on a location of Fig. $12 \mathrm{~b}$ and hence it is a $Z V$-line graph.

The combinatorial intuition, which later will help us prove our results, is captured by the following lemma.

Lemma 1 For any location $\ell \in \mathcal{V}$, the preferences of an agent located on $\ell$ and an agent located on $\mathcal{R}(\ell)$ over the locations of $\mathcal{G}_{\perp}$ are identical. That is,

$$
\forall u, v \in V_{\perp}: \quad d(\ell, u) \leqslant d(\ell, v) \Longleftrightarrow d(\mathcal{R}(\ell), u) \leqslant d(\mathcal{R}(\ell), v) .
$$

Moreover, if $\ell \in V_{i}$ for some $i \in\{1, \ldots, k\}$, then the preferences of an agent located on $\ell$ and an agent located on $\mathcal{R}(\ell)$ over the locations outside of $V_{i} \backslash V_{\perp}$ are identical. That is,

$$
\forall u, v \notin V_{i} \backslash V_{\perp}: \quad d(\ell, u) \leqslant d(\ell, v) \Longleftrightarrow d(\mathcal{R}(\ell), u) \leqslant d(\mathcal{R}(\ell), v) .
$$

Corollary 1 For any location $\ell \in \mathcal{V}$, if $\ell \in V_{i}$ for some $i \in\{1, \ldots, k\}$, then an agent located on $\ell$ strictly prefers $\mathcal{R}(\ell)=\mathcal{R}\left(\mathcal{G}_{i}\right)$ over any other location outside of $V_{i}$.

Given a non-simple $Z V$-line graph $\mathcal{G}=\langle\mathcal{V}, \mathcal{E}\rangle$ w.r.t. a cover $V_{\perp}, V_{1}, \cdots, V_{k}(k \geqslant 1)$ and mechanisms $\mathcal{F}_{i}: x \in V_{i}^{\star} \rightarrow V_{i}$ for $i=\perp, 1, \ldots, k$, we define the following mechanism $\mathcal{F}_{\text {rec }}^{\star}: \mathcal{V}^{\star} \rightarrow \mathcal{V}$.

Definition $6\left(\mathcal{F}_{\text {rec }}^{\star}-A\right.$ mechanism for non-simple $Z V$-line graphs) For a non-simple $Z V$ -line graph $\mathcal{G}=\langle\mathcal{V}, \mathcal{E}\rangle$, given a ballot profile (location reports of the agents) $x \in \mathcal{V}^{\star}$, 


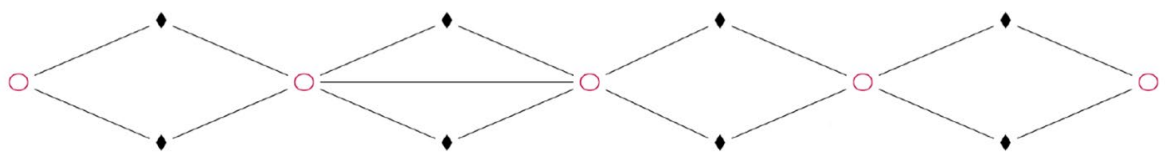

(a)

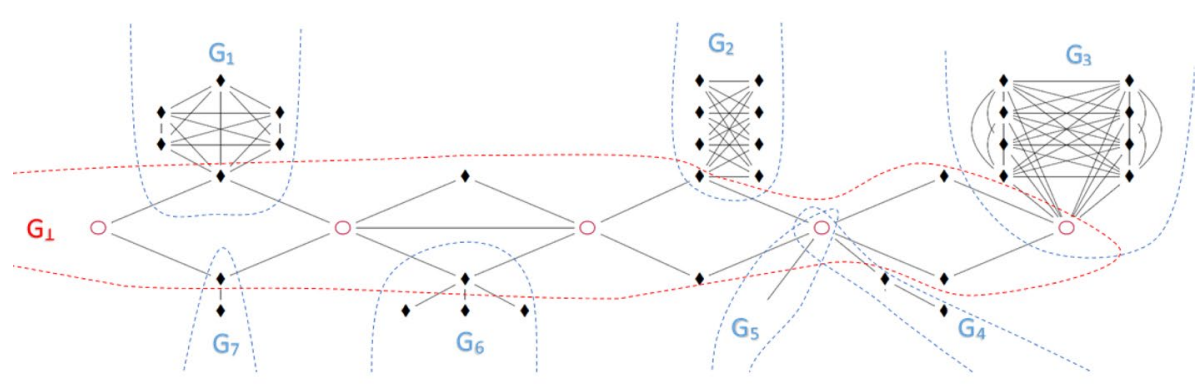

(b)

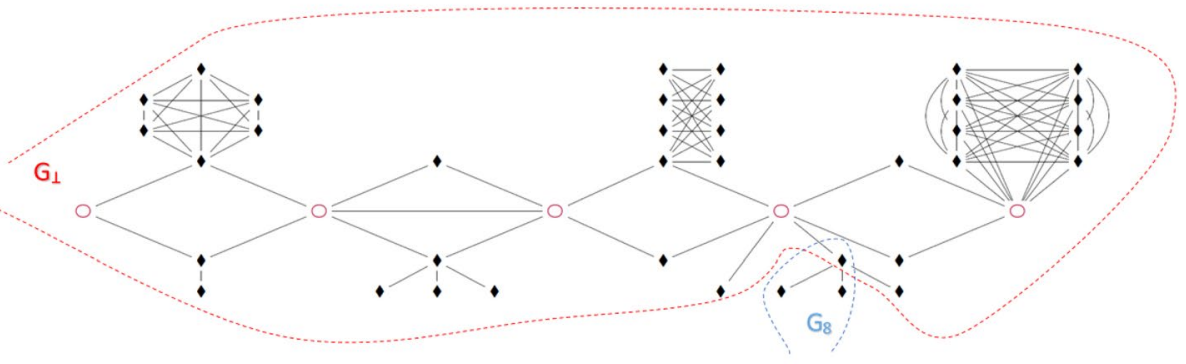

(c) The graph of Figure 7 .

Fig. 12 A construction of the graph of Fig. 7 (Color figure online)

If there exists a subgraph $\mathcal{G}_{i}$ for some $i \in\{1, \ldots, k\}$ s.t. all ballots belong to $V_{i}$, return $\mathcal{F}_{i}(\boldsymbol{x}){ }^{16}$

$>$ Otherwise, let $\mathcal{R}(\boldsymbol{x})$ be the profile generated by replacing each ballot $\ell$ by $\mathcal{R}(\ell)$, and return $\mathcal{F}_{\perp}(\mathcal{R}(\boldsymbol{x}))$. (Since $\mathcal{R}(\ell) \in V_{\perp}$ for any location $\ell \in \mathcal{V}, \mathcal{F}_{\perp}(\mathcal{R}(\boldsymbol{x})$ ) is well-defined.)

The mechanism $\mathcal{F}_{\text {rec }}^{\star}$ has a similar interpretation to the interpretation of $\mathcal{F}_{\text {sZV }}^{\star}$, with $V_{\perp}$ taking the role of the $Z$-locations: The facility is commonly positioned on a location of $\mathcal{G}_{\perp}$ (unless all agents unanimously agree on a different subgraph). For instance, $\mathcal{G}_{\perp}$ could represent commercial locations for locating a public mall, the set of status-quo outcomes, or other norms of the society.

\footnotetext{
${ }^{16}$ If there are more than one such subgraph, choose one of them arbitrarily. Note that if there are several such subgraphs, then it must be that all the ballots are identical and equal to $\mathcal{R}\left(\mathcal{G}_{i}\right)$. Since later we restrict ourselves to Pareto-optimal $\mathcal{F}_{i}$ (so $\mathcal{F}_{i}(\boldsymbol{x})=\mathcal{R}\left(\mathcal{G}_{i}\right)$ ), this arbitrary choice does not influence the outcome of $\mathcal{F}^{\star}$ rec
} 
Now, we can define our mechanism for $Z V$-line graphs, by applying Definition 6 recursively on $\mathcal{G}$ and its subgraphs (taking simple $Z V$-line graphs and $\mathcal{F}_{\text {sZV }}^{\star}$ as the base of the recursion).

Definition $7\left(\mathcal{F}^{\star}\right.$-The mechanism for $Z V$-line graphs) For a $Z V$-line graph $\mathcal{G}=\langle\mathcal{V}, \mathcal{E}\rangle$, given a ballot profile (location reports of the agents) $x \in \mathcal{V}^{\star}$,

If $\mathcal{G}$ is a simple $Z V$-line graph, return $\mathcal{F}_{\text {sZV }}^{\star}(\boldsymbol{x})$.

Otherwise, $\mathcal{G}$ is a $Z V$-line graph w.r.t. some cover $V_{\perp}, V_{1}, \cdots, V_{k}(k \geqslant 1)$.

Let $\mathcal{F}_{\text {rec }}^{\star}$ be the mechanism as defined in Definition 6 w.r.t. the cover $V_{\perp}, V_{1}, \cdots, V_{k}(k \geqslant 1)$.

$>\operatorname{Return} \mathcal{F}_{\text {rec }}^{\star}(\boldsymbol{x})$.

First, we claim that $\mathcal{F}^{\star}$ satisfies the following desired properties:

$\mathcal{F}^{\star}$ is an order-based mechanism: If $\mathcal{G}$ is a simple $Z V$-line graph, then $\mathcal{F}^{\star}=\mathcal{F}_{\text {sZV }}^{\star}$ which we already saw is an order-based mechanism.

If $\mathcal{G}$ is a non-simple $Z V$-line graph, then $\mathcal{F}^{\star}=\mathcal{F}_{\text {rec }}^{\star}$ and we claim that $\mathcal{F}_{\text {rec }}^{\star}$ is an orderbased mechanism whenever the mechanisms $\mathcal{F}_{\perp}, \mathcal{F}_{1}, \ldots, \mathcal{F}_{k}$ are order-based mechanisms (of the respective graphs). This property holds since

- If $P O(x) \cap V_{\perp} \neq \emptyset$, then by Lemma $1, P O(x) \cap V_{\perp}=P O(\mathcal{R}(x)) \cap V_{\perp}$.

- There is no Pareto-optimal location in $\mathcal{G}_{\perp}$, only if all the ballots belong to a sub-graph $\mathcal{G}_{i}$ for some $i \in\{1, \ldots, k\}$.

- For any $i \in\{1, \ldots, k\}, P O(\boldsymbol{x}) \cap\left(V_{i} \backslash V_{\perp}\right) \neq \emptyset$ only if all the ballots belong to $\mathcal{G}_{i}$.

- For any $i \in\{\perp, 1, \ldots, k\}, \mathcal{R}\left(\mathcal{G}_{i}\right)$ must be the first location in the order of $\mathcal{F}_{i}$, since by the definition of the mechanism when all locations of $\mathcal{G}_{i}$ are voted for, the outcome is $\mathcal{R}\left(\mathcal{G}_{i}\right)$.

Hence, an equivalent order-based definition of $\mathcal{F}_{\text {rec }}^{\star}$ is returning the first Pareto-optimal location in the following order: First, go over the locations in $\mathcal{G}_{\perp}$ and then iterate over the locations of the other subgraphs, while for each subgraph $\mathcal{G}_{i}$ iterate over its locations according to the order of $\mathcal{F}_{i}$.

$\mathcal{F}^{\star}$ can be computed in polynomial time: If $\mathcal{G}$ is a simple $Z V$-line graph, then $\mathcal{F}^{\star}=\mathcal{F}_{\text {sZV }}^{\star}$ which we already saw can be computed in polynomial time.

Otherwise, it is enough to notice that by Definition 6 , if $\mathcal{F}_{\perp}, \mathcal{F}_{1}, \ldots, \mathcal{F}_{k}$ can be computed in polynomial time, then $\mathcal{F}_{\text {rec }}^{\star}$ can be computed in polynomial time. So, the time complexity of $\mathcal{F}^{\star}$ can be bounded via a recursive argument.

Our main result shows that $\mathcal{F}^{\star}$ satisfies the desired efficiency and manipulation-resistance properties.

Theorem 1 (Main result) Let $\mathcal{G}=\langle\mathcal{V}, \mathcal{E}\rangle$ be a $Z V$-line graph and let $\mathcal{F}^{\star}: \mathcal{V}^{\star} \rightarrow \mathcal{V}$ be the corresponding mechanism as defined in Definition 7. Then $\mathcal{F}^{\star}$ is an anonymous, Paretooptimal, group-manipulation-resistant mechanism.

We prove the theorem by structural induction over the set of $Z V$-line graphs. We already proved the base case of simple $Z V$-line graphs (Proposition 2). The main ingredient in the 
proof is showing that these desired properties are elevated by the recursive construction of $\mathcal{F}_{\text {rec }}^{\star}$ (Definition 6).

Proposition 7 Let $\mathcal{G}=\langle\mathcal{V}, \mathcal{E}\rangle$ be an unweighted undirected connected graph with a non-trivial cover $V_{\perp}, V_{1}, \cdots, V_{k}(k \geqslant 1)$, and denote by $\mathcal{G}_{i}$ the subgraph $\left\langle\mathcal{V}=V_{i}, \mathcal{E}=\mathcal{E} \cap\left(\begin{array}{c}V_{i} \\ 2\end{array}\right)\right\rangle$, s.t.

- For $i=1, \ldots, k$, there exists a unique location in $V_{i} \cap V_{\perp}$, which we denote by $\mathcal{R}\left(\mathcal{G}_{i}\right)$.

- For $i=1, \ldots, k$, there are no edges between locations in $\left(V_{i} \backslash\left\{\mathcal{R}\left(\mathcal{G}_{i}\right)\right\}\right)$ and locations outside of $V_{i}$. (Equivalently, all paths between locations of $V_{i}$ and locations outside of $V_{i}$ include the root $\mathcal{R}\left(\mathcal{G}_{i}\right)$ ).

- The sets $\left\{V_{i} \backslash\left\{\mathcal{R}\left(\mathcal{G}_{i}\right)\right\}\right\}_{i=1, \ldots, k}$ are pairwise disjoint.

For $i=\perp, 1, \ldots, k$, let $\mathcal{F}_{i}: x \in V_{i}^{\star} \rightarrow V_{i}$ be a mechanism for $\mathcal{G}_{i}$ s.t.

- $\mathcal{F}_{i}$ is an anonymous, Pareto-optimal, group-manipulation-resistant mechanism.

- For an infinite number of $\tau \in \mathbb{N}$, there exists a profile $\boldsymbol{x} \in V_{i}^{\star}$ in which there are at least $\tau$ ballots for any location in $V_{i}$ s.t. $\mathcal{F}_{i}(\boldsymbol{x})=\mathcal{R}\left(\mathcal{G}_{i}\right)$.

Then, for $\mathcal{F}_{\text {rec }}^{\star}: \mathcal{V}^{\star} \rightarrow \mathcal{V}$ as defined in Definition 6 ,

- $\mathcal{F}_{\text {rec }}^{\star}$ is an anonymous, Pareto-optimal, group-manipulation-resistant mechanism.

- For any number $\tau \in \mathbb{N}$, there exists a profile $x \in \mathcal{V}^{\star}$ in which there are at least $\tau$ ballots for any location in $\mathcal{V}$ s.t. $\mathcal{F}_{\text {rec }}^{\star}(\boldsymbol{x})=\mathcal{R}(\mathcal{G})=\mathcal{R}\left(\mathcal{G}_{\perp}\right)$.

Note that we do not require the subgraphs $V_{\perp}, V_{1}, \cdots, V_{k}$ to necessarily be $Z V$-line graphs. This allows us to apply the recursive construction also for graphs for which an anonymous, Pareto-optimal, group-manipulation-resistant mechanism is known but are not $Z V$-line graphs (e.g., $C_{5}$ ). We prove Proposition 7 in Appendix C. In fact, we prove a more robust result (Proposition 12) showing that also weaker notions of manipulation-resistance can be lifted from the mechanisms for the subgraphs $\mathcal{G}_{i}$ to the mechanism $\mathcal{F}^{\star}$, e.g., resistance against manipulations of only some coalitions and resistance against only some types of manipulations.

Proof of Theorem 1 We prove the following using structural induction over the recursive definition of $Z V$-line graphs (Definition 5).

- $\mathcal{F}^{\star}$ is an anonymous Pareto-optimal mechanism.

- $\mathcal{F}^{\star}$ is a group-manipulation-resistant mechanism. That is, for any location profile $\boldsymbol{x} \in \mathcal{V}^{\star}$, a coalition of agents $C$, and a set of ballots $\boldsymbol{A} \in \mathcal{V}^{\star}, \boldsymbol{A}$ is not a beneficial deviation for $C$.

- For any number $\tau \in \mathbb{N}$, there exists a profile $x \in \mathcal{V}^{\star}$ in which there are at least $\tau$ ballots for any location in $\mathcal{V}$ s.t. $\mathcal{F}^{\star}(\boldsymbol{x})=\mathcal{R}(\mathcal{G})$.

Base (Simple $Z V$-line graphs): When $\mathcal{G}$ is a simple $Z V$-line graph, we showed in Proposition 2 that $\mathcal{F}_{\mathrm{sZV}}^{\star}$ is an anonymous, Pareto-optimal, group-manipulation-resistant mechanism. Given $\tau \in \mathbb{N}$, consider the location profile $\boldsymbol{x}$ in which each location in $\mathcal{V}$ appears in exactly $\tau$ 
ballots. Then, $P O(\boldsymbol{x})=\mathcal{V}$ and by Definition $4, \mathcal{F}_{\mathrm{sZV}}^{\star}(\boldsymbol{x})$ is the leftmost $Z$-location of $\mathcal{G}$, which is $\mathcal{R}(\mathcal{G})$.

Step (Non-simple $Z V$-line graphs): When $\mathcal{G}$ is a non-simple $Z V$-line graph, Proposition 7 gives us that whenever the respective mechanisms $\mathcal{F}_{i}$ for the subgraphs $\mathcal{G}_{i}$ for $i=\perp, 1, \ldots, k$ are anonymous, Pareto-optimal, group-manipulation-resistant mechanisms and for an infinite number of $\tau \in \mathbb{N}$, there exists a profile $x \in V_{i}^{\star}$ in which there are at least $\tau$ ballots for any location in $V_{i}$ s.t. $\mathcal{F}_{i}(\boldsymbol{x})=\mathcal{R}\left(\mathcal{G}_{i}\right)$, the $\mathcal{F}^{\star}$ also satisfies the above desired properties.

\subsection{Recursive families of $Z V$-line graphs}

Many graph families are defined using a recursive definition: That is, stating a base case that comprises an initial (and commonly small) family of simple graphs and an inductive step defining graphs in the family as a simple amalgamation of other (more basic) graphs in the family (for example, the second case of the definition of $Z V$-line graphs, Definition 5). Given a recursive family of $Z V$-line graphs, the mechanism $\mathcal{F}^{\star}$ of Theorem 1 is a recursive, and hence commonly simple, mechanism that satisfies our desiderata.

Example (i): Rooted trees A simple example of a recursive family of $Z V$-line graphs is rooted trees which can be defined recursively as follows (See also Fig. 13):

Base: A tree of height 0 is a single location (and it is also the root of the tree).

Step: A tree of height $h>0$ consists of a location (the root) which is connected to the roots of a non-empty set of trees s.t. the maximal height of them equals $(h-1)$.

We can see that indeed these graphs are $Z V$-line graphs, since a tree of height 0 (single location) and trees of height 1 (bicliques) are simple $Z V$-line graphs and the recursive step of the definition satisfies the recursive connectivity constraint of Definition 5. Hence, we get, as a corollary of Theorem 1, that the mechanism that returns the lowest common ancestor of the ballots is an anonymous, Pareto-optimal, group-manipulation-resistant mechanism. Noting that given a tree graph, it is a rooted graph w.r.t. the root being any of the locations, we get that any mechanism $\mathcal{F}$ that returns the lowest common ancestor of the ballots w.r.t. some arbitrary root $r$, or equivalently

$$
\mathcal{F}(\boldsymbol{x})=\underset{v \in P O(x)}{\operatorname{argmin}} d(v, r),
$$
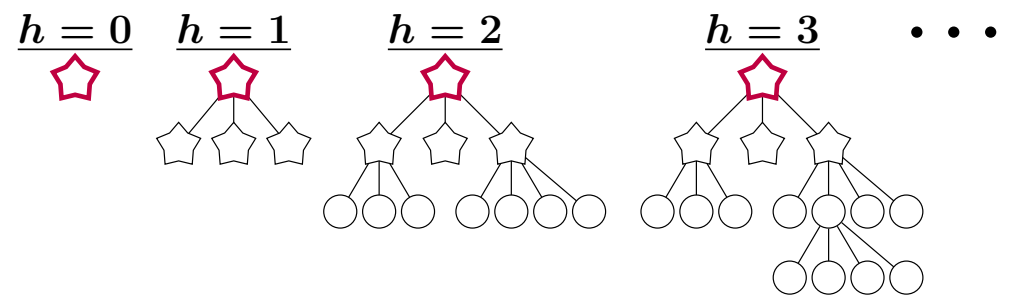

Fig. 13 Trees of height $0,1,2$ and 3. The new location (the root) added in each step is denoted by $\square$. The

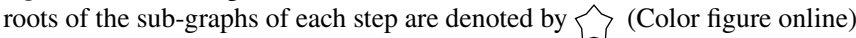



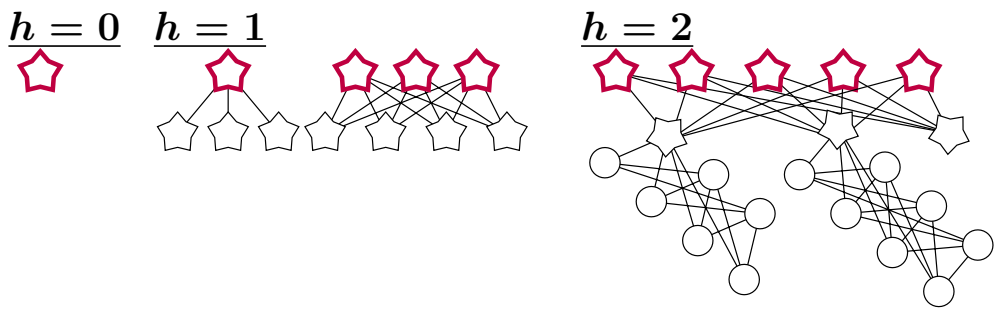

Fig. 14 Graphs of complexity 0,1 and 2. The new pre-roots added in each step are denoted by $\underline{0}$. The

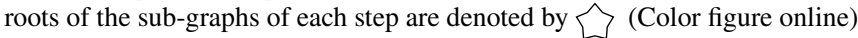

is an anonymous, Pareto-optimal, group-manipulation-resistant mechanism. These are also the mechanisms that Todo et al. [67] characterized as the false-name-proof, anonymous, Pareto-optimal mechanisms for the continuous tree.

Example (ii): A generalization of rooted trees We show an anonymous, Pareto-optimal, group-manipulation-resistant mechanism for the following family of rooted graphs (that is, $\langle\mathcal{V}, \mathcal{E}, r\rangle$ s.t. $\mathcal{E} \subseteq\left(\begin{array}{l}\mathcal{V} \\ 2\end{array}\right)$ and $r \in \mathcal{V}$ ). (See also Fig. 14)

\section{Definition $8(\mathcal{C})$}

Base: $\langle\{v\}, \emptyset, v\rangle \in \mathcal{C}$.

Step: For any $k, \ell \geqslant 1$ : If $\left\{\left\langle\mathcal{V}_{i}, \mathcal{E}_{i}, r_{i}\right\rangle\right\}_{i=1}^{k}$ are graphs in $\mathcal{C}$ (and the $\mathcal{V}_{i}$ are pairwise disjoint), then also the graph

$$
\left\langle\left\{\hat{r}_{j}\right\}_{j=1}^{\ell} \dot{\cup}\left(\bigcup_{i=1}^{k} \mathcal{V}_{i}\right),\left(\bigcup_{i=1}^{k} \mathcal{E}_{i}\right) \dot{\cup}\left\{\left(\widehat{r}_{j}, r_{i}\right)\right\}_{\begin{array}{l}
i=1 \ldots k \\
j=1 \ldots \ell
\end{array}}, \widehat{r}_{1}\right\rangle
$$

is in $\mathcal{C}$. That is, adding a new layer of $\ell$ pre-roots, a biclique between the pre-roots and the roots of the sub-graphs, and defining the new root to be one of the pre-roots.

We use the notation $h(\mathcal{G})$ for the minimal number of steps needed to generate $\mathcal{G}$ and call it the complexity of $\mathcal{G}$.

We note that the graphs of complexity $h(\mathcal{G})=1$ are the bicliques and that by taking $\ell=1$ in the recursive step of the definition we get the family of rooted trees. Hence, both are sub-families of this family of graphs. We can see that indeed these graphs are $Z V$-line graphs by noticing that the recursive step of the definition satisfies the recursive connectivity constraint of Definition 5.

Our mechanism for these graphs:

Find the subgraph $G^{\prime}$ of lowest complexity s.t. all ballots belong to $G^{\prime}$.

If there exists a ballot for a pre-root of $G^{\prime}$, the mechanism returns the leftmost preroot of $G^{\prime}$ that was voted for.

Otherwise, the mechanism returns the leftmost pre-root of $G^{\prime}$. 
Notice that the order over the pre-roots is arbitrary, so different mechanisms could arise from different choices of orders and mechanisms for $\mathcal{G}_{\perp}$ (For instance, the order might represent the social norm of the society). ${ }^{17}$

Example (iii): Block graphs Our last example is connected block graphs [33]. ${ }^{18}$

Definition 9 (Connected block graphs) A connected graph $\mathcal{G}=\langle\mathcal{V}, \mathcal{E}\rangle$ is a block graph if the following equivalent conditions hold:

- Every biconnected subgraph of $\mathcal{G}$ is a clique. ${ }^{19}$

- The intersection of any two connected subgraphs of $\mathcal{G}$ is either empty or connected.

- For every four vertices $u, v, w, x \in \mathcal{V}$, the largest two of the three distance sums

$$
d(u, v)+d(w, x), \quad d(u, w)+d(v, x), \text { and } \quad d(u, x)+d(v, w)
$$

are equal.

In general, any connected graph $\mathcal{G}$ decomposes into a tree of biconnected components called the block-cut tree of the graph. The block-cut tree of a graph $\mathcal{G}$ is a tree $\mathcal{T}(\mathcal{G})$ that is defined in the following way. In $\mathcal{T}(\mathcal{G})$ there is a vertex (component-vertex) for each maximal biconnected component of $\mathcal{G}$ and a vertex (intersection-vertex) for each vertex in $\mathcal{G}$ that belongs to more than one maximal biconnected component. There is an edge in $\mathcal{T}(\mathcal{G})$ between each component-vertex and the intersection-vertices belonging to this component.

Hence, since for connected block graphs all maximal biconnected components are cliques, a connected block graph $\mathcal{G}$ can be represented by its block-cut tree $\mathcal{T}(\mathcal{G})$ s.t. each component-vertex is labeled by the size of the represented clique and each intersection-vertex is labeled by the indices of the represented vertex in the respective cliques. Moreover, any such labeled tree defines a (unique) block graph. ${ }^{20}$

Given a block graph $\mathcal{G}$, its block-cut tree $\mathcal{T}(\mathcal{G})$ induces a recursive structure decomposing $\mathcal{G}$ to smaller block graphs. Our mechanism is defined w.r.t. a choice of an arbitrary

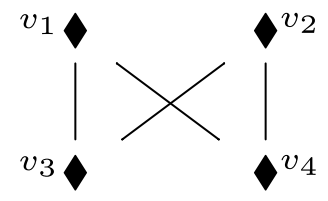

Fig. 15 The (2,2)-biclique

\footnotetext{
17 In the version of this work that appeared on AAMAS [49, Claim 3.16] we erroneously claimed that the mechanism $\mathcal{F}(\boldsymbol{x})=\operatorname{argmin}_{v \in P O(x)} d(v, r)$, that returns the Pareto-optimal location closest to the root and breaks ties according to some predefined order is a group-manipulation-resistant mechanism. A counterexample for this claim is the (2,2)-biclique (see Fig. 15). Assume $\mathcal{F}$ returns the Pareto-optimal location closest to $v_{3}$ and consider the profile $\left\langle v_{1}, v_{2}, v_{4}\right\rangle$. Then the Pareto-optimal locations are $\left\{v_{1}, v_{2}, v_{4}\right\}$ and the Pareto-optimal locations closest to $v_{3}$ are $v_{1}$ and $v_{2}$. Assume that the mechanism returns $v_{1}$ (and the case of $v_{2}$ is symmetric). Then the agent located on $v_{2}$ can manipulate by changing her vote to $v_{3}$ and changing the outcome to be $v_{3}$ which is closer to her.

18 We thank Ayumi Igarashi for suggesting us this family as an example.

19 A graph is biconnected if it is a connected graph that is not broken into disconnected pieces by deleting any single vertex (and its incident edges). An equivalent definition is that a graph is biconnected if, for every pair of its vertices, it is possible to find two vertex-independent paths connecting these two vertices.

20 This is the reason connected block graphs are also called clique trees.
} 
root of $\mathcal{T}(\mathcal{G})$ and arbitrary orders for every clique over its locations. We can see that indeed these graphs are $Z V$-line graphs, by noting that cliques are simple $Z V$-line graphs and that the recursive step of the definition satisfies the recursive connectivity constraint of Definition 5.

Our mechanism for a connected block graph $\mathcal{G}$ is:

Find the component $G^{\prime}$ that is represented by the lowest common ancestor of the ballots.

$\checkmark$ If one of the locations of $G^{\prime}$ was voted for, the mechanism returns the first location of $G^{\prime}$ (according to the order) that was voted for.

$>$ Otherwise, the mechanism returns the first location of $G^{\prime}$.

An equivalent definition of this family of mechanisms is returning the closest Pareto-optimal location to some arbitrary location $v$, breaking ties according to an arbitrary fixed order.

\section{Discussion}

In this work, we presented a new family of graphs, $Z V$-line graphs, and a generic, anonymous, Pareto-optimal, group-manipulation-resistant mechanism for the facility location problem on these graphs (Theorem 1). The mechanism $\mathcal{F}^{\star}$ we presented is not the only mechanism satisfying the desired properties. For instance, a mechanism that outputs at the second stage of Definition 4 the rightmost Pareto-optimal Z-location instead of the leftmost would also satisfy the desiderata. Generally, taking any order over the $Z$-locations of a simple $Z V$-line graph s.t. the constraints of Definition 3 hold and defining $\mathcal{F}_{\text {sZV }}^{\star}$ accordingly will satisfy them. As we saw on Claim 4, there exists an anonymous, Pareto-optimal, group-manipulation-resistant mechanism for the cycle of size four, which is a simple $Z V$ -line graph which is not of the template of Definition 4. On the other hand, we saw in Corollary 2 that for any anonymous, Pareto-optimal, group-manipulation-resistant mechanism $\mathcal{H}$ for the cycle of size four, it holds that all agents are ex-post indifferent between $\mathcal{H}$ and a mechanism $\mathcal{F}$ that is derived by Definition 4 . Likewise, also the non-order-based mechanism for the (2,3)-biclique in Claim 3 is equivalent in this ex-post indifference sense to a mechanism that is derived by Definition 4 . We suspect this is also true in general and conjecture that for any $Z V$-line graph $\mathcal{G}$, the only anonymous, Pareto-optimal, group-manipulation-resistant mechanisms for $\mathcal{G}$ are either derived by Definition 4 or equivalent (in this ex-post indifference sense) to a mechanism which is.

Furthermore, unifying the non-existence results we had found, we think that the partition into $Z$-locations and $V$-locations is a fundamental property of a false-name-proof mechanism. The only non- $Z V$-line graphs for which we found an anonymous, Paretooptimal, group-manipulation-resistant mechanism are the cycle of size 5 (See Sect. 6) and graphs derived from it by Proposition 7, e.g., the graph of Fig. 16.

We conjecture that the cycle of size 5 is a representative extreme exception and that except for very few small graphs, there are anonymous, Pareto-optimal, group-manipulation-resistant graphs only for $Z V$-line graphs. 


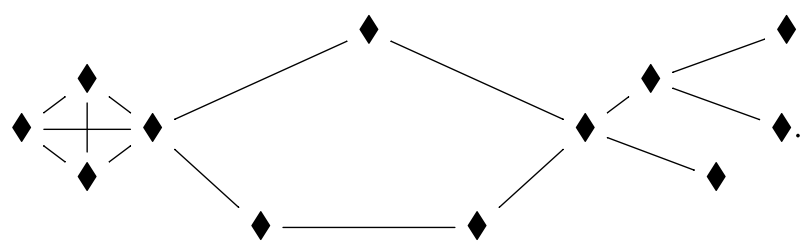

Fig. $16 C_{5}$ with a 4-clique planted on its left vertex and a tree planted on its right vertex

Conjecture 1 Let $\mathcal{G}=\langle\mathcal{V}, \mathcal{E}\rangle$ be a graph and let $\mathcal{F}: \mathcal{V}^{\star} \rightarrow \mathcal{V}$ be an anonymous, Paretooptimal, group-manipulation-resistant mechanism for $\mathcal{G}$. Then, except for few small graphs, if $\mathcal{F}$ is an order-based mechanism, then one of the following two cases holds

(a) $\mathcal{G}$ is a simple $Z V$-line graph w.r.t. some partition of $\mathcal{V}$ to $V$-locations and $Z$-locations and $\mathcal{F}$ is the outcome of applying Definition 4.

(b) There exists a non-trivial cover $V_{\perp}, V_{1}, \cdots, V_{k} \subseteq \mathcal{V}$ of $\mathcal{V}$ s.t.

- $\mathcal{G}$ is a $Z V$-line graph w.r.t. the cover $V_{\perp}, V_{1}, \cdots, V_{k}$;

- For $i=\perp, 1, \ldots, k$ : Whenever $\boldsymbol{x} \in\left(V_{i}\right)^{\star}$, i.e., all locations are in $V_{i}$, also $\mathcal{F}(\boldsymbol{x}) \in V_{i}$

- The mechanisms $\mathcal{F}_{i}:\left(V_{i}\right)^{\star} \rightarrow V_{i}^{\star}$ that are defined by $\mathcal{F}_{i}(\boldsymbol{x})=\mathcal{F}(\boldsymbol{x})$ are anonymous, Pareto-optimal, group-manipulation-resistant mechanisms, for $i=\perp, 1, \ldots, k$; and

- $\mathcal{F}$ is the outcome of applying Definition 6 for the mechanisms $\mathcal{F}_{i}$.

On the other hand, if $\mathcal{F}$ is not an order-based mechanism, then there exists an order-based mechanism $\mathcal{H}$ s.t. all agents are ex-post indifferent between $\mathcal{F}$ and $\mathcal{H}$ (Hence, $\mathcal{H}$ is an anonymous, Pareto-optimal, group-manipulation-resistant mechanism for $\mathcal{G}$ too, and the first case holds for $\mathcal{H}$ ).

Consequentially, showing that a given graph does not have such a structure could be an easy and efficient way to prove the non-existence of an anonymous, Pareto-optimal, groupmanipulation-resistant mechanism. The conjecture also implies that all manipulation-resistant anonymous Pareto-optimal mechanisms (up to the above equivalence) are computationally easy to run.

\subsection{Approximate mechanism design without money}

Assuming Conjecture 1, one gets that whenever there is a large disagreement in the population (i.e., the agents are dispersed over many $V_{i}$-subgraphs), an extreme status-quo alternative must be chosen by the mechanism. This raises the natural question of how much efficiency must be sacrificed by requiring manipulation-resistance. That is, assuming the agents are quantitatively represented by a cost function (e.g., the distance to the facility or a monotone function of the distance) and analyzing the implications of manipulation-resistance on the approximability of the minimization problem of natural social cost functions, e.g., the average cost (Harsanyi social welfare), the geometric mean of the costs (similar to the Nash social welfare), or the maximum cost (Rawls' criterion).

Assuming Conjecture 1 results in a high price of false-name-proofness for most nontrivial graphs (of the order of the number of agents times the diameter of the graph). For comparison, both the mechanism that always outputs a constant location and the random 
mechanism that outputs one of the ballots at random have a price of false-name-proofness of the same order. Both mechanisms, although they are not deterministic false-name-proofness Pareto-optimal mechanisms and as such are out of the scope of this work, are very simplistic and clearly could not be seen as optimizing social welfare. As we show below, this phenomenon of losing a large portion of performance due to requiring false-name-proofness is not unique to facility location problems, and also in other domains, one could get highly simplistic mechanisms with the same performance as any false-name-proof mechanism. In a sense, this is similar to the impossibility results for unconstrained voting rules of Gibbard [30] and Satterthwaite [59] for deterministic rules, and of Gibbard [31] for random voting rules.

We think this should not be surprising. The false-name-proofness property requires that an agent should not benefit even from casting a colossal number of identical ballots, ${ }^{21}$ and hence the designer must act as if any of the ballots represents the vast majority of society. So when the ballots are dispersed enough, from the viewpoint of the designer, all locations guarantee roughly the same social value (for most natural notions of social value).

False-name-proof combinatorial auctions: Yokoo et al. [79] showed in their work that there is no false-name-proof mechanism for combinatorial auctions which allocates the resources efficiently, and later results ([54]) showed that false-name-proofness is impossible even under a weaker maximality constraint. Following this work, Iwasaki et al. [36] showed that there is no symmetric false-name-proof mechanism (which also satisfies some apparently minor assumptions) that is guaranteed to get more than $\frac{2}{m+1}$ of the optimal social welfare, for $m$ being the number of resources and there are strictly more agents than resources, even when all bidders are single-minded ([36, Thm. 2]). On the other hand, this efficiency approximation is achieved by a trivial mechanism that auctions the grand bundle in a second-price auction ([36, Thm. 3]), in particular, ignoring most of its input and the combinatorial aspect of the scenario.

False-name-proof voting scenarios: In general voting settings, Conitzer [15] showed that all neutral anonymous false-name-proof voting rules must have a non-negligible random component (and in particular could not be deterministic) and in many scenarios must be far from maximizing any reasonable quantitative efficiency criterion. For instance,

- Unless there is unanimity among the voters on some pair of alternatives, any falsename-proof voting rule must choose the winner uniformly at random. This holds even when there are only two alternatives.

- If there are at least three alternatives, then under any false-name-proof voting rule, for any profile, the probability of any given alternative to win is at most $2 / \mathrm{m}$. Moreover, even when all agents top-rank the same alternative and even when all agents hold the same preference, there is no false-name-proof voting rule which outputs this top-ranked alternative with probability higher than $2 / m$.

False-name-proof facility-location on the continuous line: Todo et al. [67] showed that any false-name-proof facility-location mechanism for the continuous line could not guarantee to get the sum of agents' costs below $C / n$ of the optimal, for $n$ being the number of agents and $C$ being some (mechanism-dependent) constant. In this work, they also showed that there is no false-name-proof facility-location mechanism for the

${ }^{21}$ Which is equivalent to resistance to casting one extra ballot, as we saw in Proposition 1. 
continuous line that guarantees to get the maximum cost of an agent below $1 / 2$ of the optimal. On the other hand, they showed that both approximability bounds are achieved by the false-name-proof mechanism that outputs the leftmost ballot.

\section{The discrete cycle over $\boldsymbol{n}$ locations}

In this section, we characterize the discrete cycle graphs over $n$ locations, $C_{n}$, for which a group-manipulation-resistant, anonymous, Pareto-optimal mechanism exists. In particular, we show that for a large enough cycle $(n \geqslant 6)$, there is no anonymous Pareto-optimal mechanism that is resistant even to manipulations of a single agent. On the other hand, we show that for smaller cycles, one can construct group-manipulation-resistant, anonymous, Pareto-optimal mechanisms.

$\boldsymbol{C}_{2}, \boldsymbol{C}_{\mathbf{3}}, \boldsymbol{C}_{\mathbf{4}}$ : These three graphs are $Z V$-line graphs: $C_{2}$ and $C_{3}$ are cliques and hence can be defined as $Z V$-line graphs with only $Z$-locations (and any order over them), $C_{4}$ is a $(2,2)$ -biclique and hence can be defined as a $Z V$-line graph w.r.t. taking two non-adjacent locations to be the $Z$-locations (and any order over them) and two singleton $V_{i}$-subgraphs consisting of the other two locations. Moreover, we show that there are no other mechanisms for $C_{2}$ and $C_{3}$ and that all other mechanisms for $C_{4}$ are in some sense equivalent to the above mechanism. (The proofs of the following propositions can be found in Appendix D).

\section{Proposition 8}

- The only false-name-proof, anonymous, Pareto-optimal mechanisms for $C_{2}$ are the two order-based mechanisms.

- The only false-name-proof, anonymous, Pareto-optimal mechanisms for $C_{3}$ are the six order-based mechanisms.

Proposition $9 \mathcal{F}$ is a false-name-proof, anonymous, Pareto-optimal mechanism for $C_{4}$ iff there exists a cyclic labeling of the graph by $\{a, b, c, d\}$ (see Fig. 17) s.t. for any profile $x \in \mathcal{V}^{\star}$

- If all ballots are identical and equal to a location $\ell$, then $\mathcal{F}(\boldsymbol{x})=\ell$.

- If $a \in \boldsymbol{x}$, then $\mathcal{F}(\boldsymbol{x})=a$.

- If $a \notin \boldsymbol{x}$ and $c \in \boldsymbol{x}$, then $\mathcal{F}(\boldsymbol{x})=c$.

- If $\boldsymbol{x}$ includes ballots for both location $b$ and location $d$ and only these two locations, then $\mathcal{F}(\boldsymbol{x}) \in\{a, c\}$.

Corollary 2 For any two false-name-proof, anonymous, Pareto-optimal mechanisms for $C_{4}$ using the same labeling, $\mathcal{F}$ and $\mathcal{H}$ as above, all agents are ex-post indifferent between the two mechanisms. That is, for any profile $\boldsymbol{x} \in \mathcal{V}^{\star}$, all agents are indifferent between the two locations $\mathcal{F}(\boldsymbol{x})$ and $\mathcal{H}(\boldsymbol{x})$.

Proof The only scenario in which $\mathcal{F}$ and $\mathcal{H}$ differ is when $\boldsymbol{x}$ includes ballots for both location $b$ and location $d$ and only these two locations. Any agent who is located in one of these two location is indifferent between location $a$ and location $c$. 


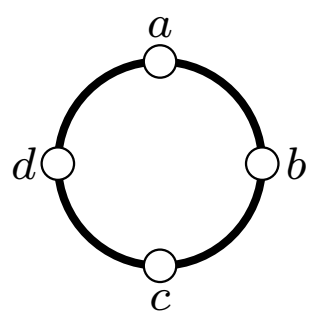

Fig. 17 The labeling of Proposition 9

$\boldsymbol{C}_{\mathbf{5}}$ : It is not hard to verify that a mechanism that returns the first Pareto-optimal location according to one of the following orders $-{ }_{3}$ manipulation-resistant mechanism. These three mechanisms are also order-based mechanisms. In Appendix D, we prove that in fact these are the only group-manipulation-resistant, anonymous, Pareto-optimal mechanisms for $C_{5}$.

Proposition 10 The only false-name-proof, anonymous, Pareto-optimal mechanisms for $C_{5}$ are the following 30 order-based mechanisms (see Fig. 18):

- $a \rightarrow b \rightarrow e \rightarrow c \rightarrow d$,

- $a \rightarrow b \rightarrow e \rightarrow d \rightarrow c$

- $a \rightarrow b \rightarrow d \rightarrow e \rightarrow c$,

- $\quad$ and their rotations and reflections.

Corollary $3 C_{5}$ is not a simple $Z V$-line graph.

Proof By Definition 4, if $C_{5}$ was a simple $Z V$-line graph, then there would be an orderbased group-manipulation-resistant mechanism for it with the $Z$-location in the prefix of the order. But, choosing any of the five location prefixes of the orders in Proposition 10 to be the Z-locations violates the constraints of Definition 3.

\section{$6.1 C_{n}$ for $n \geqslant 6$}

Proposition 11 For $n \geqslant 6$ there is no anonymous Pareto-optimal mechanism for $C_{n}$ that is resistant even only to manipulations of a single agent.

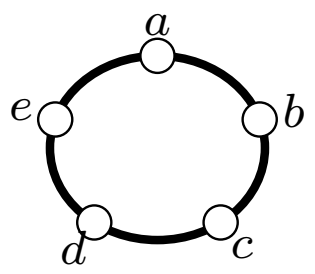

Fig. 18 The labeling of Proposition 10 


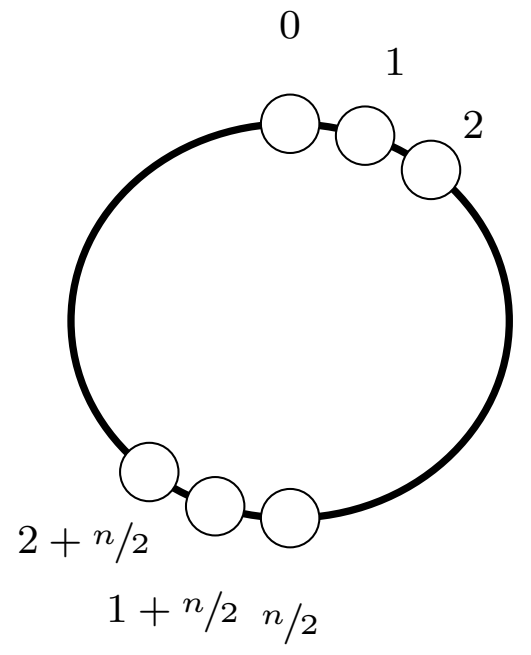

Fig. 19 Illustration for the proof of Proposition 11 for cycles of even size larger than 6

The proof generalizes the proof for $C_{6}$ which we showed on Proposition 3 . We show below the proof for large cycles of even size. The proof for the odd-sized cycles (in which a more delicate rounding in needed in the statements) can be found in Appendix D.

Proof (for cycles of even size $n \geqslant 6$ ) Assume towards a contradiction that $\mathcal{F}$ is a Paretooptimal, anonymous, manipulation-resistant mechanism for $C_{n}$. We denote the locations of $C_{n}$ by $\{0,1,2, \ldots, n-1\}$, and w.l.o.g. assume that for the profile of $n$ agents who vote for all $n$ locations the outcome is 0 (see Fig. 19).

For the profile $\left\langle 2,1+\frac{n}{2}, 2+\frac{n}{2}\right\rangle$ : From resistance to false-name manipulations of the first and last agents, the outcome must be either 0 or 4 (Since any of them can change the result to be 0 by adding false-ballots). From the Pareto-optimality of $\mathcal{F}$, the outcome cannot be 0 which is Pareto-dominated by 4 . Hence, the outcome for the profile $\left\langle 2,1+\left\lfloor\frac{n}{2}\right\rfloor, 2+\left\lfloor\frac{n}{2}\right\rfloor\right\rangle$ is 4 .

Similarly, for the profile $\left\langle\mathbf{1}, \mathbf{2}, \mathbf{1}+\frac{n}{2}\right\rangle$ the outcome must be 2 . From false-name-resistance of $\mathcal{F}^{\star}$, the outcome for the profile $\left\langle 2,1+\frac{n}{2}\right\rangle$ must also be 2 (Otherwise, the first agent can cast an additional false-ballot 1 to get the outcome to be 2).

But, the second agent in the profile $\left\langle 2,1+\frac{n}{2}\right\rangle$ (who is located on $1+\frac{n}{2}$ ) can change the outcome to be 4 which is closer to her by casting one additional false-ballot $2+\frac{n}{2}$. So we get a contradiction.

\section{Summary and future work}

In this work, we presented a new family of graphs, $Z V$-line graphs, and a generic, anonymous, Pareto-optimal, group-manipulation-resistant mechanism for the facility location problem on these graphs (Theorem 1). To the best of our knowledge, the (very few) 
false-name-proof mechanisms that were previously known were tailored for specific graphs, and this work is the first to show a generic false-name-proof mechanism for a large family, utilizing a broad graph property and unifying all existence results that we are aware of. The construction of the mechanism is recursive (Definition 6): We derive a mechanism for a given $Z V$-line graph from mechanisms for its subgraphs (which might not be $Z V$-line graphs). Hence, it is straightforward to derive from our construction general mechanisms for recursive graph families (as exemplified in Sect. 4.1).

\subsection{Relaxing the assumptions of the model}

Three assumptions we had are connectivity of the graph, a finite number of agents, and a finite number of locations. One could define mechanisms for disconnected graphs, infinite graphs, or an infinite number of agents (both countable and uncountable) as well. The definitions of the desiderata extend naturally to deal with these scenarios (while constraining the profiles, manipulations, coalitions to be measurable functions or sets).

For disconnected graphs, if each connected component is a $Z V$-line graph, the following mechanism generalizes $\mathcal{F}^{\star}$ and it satisfies the desiderata:

$\checkmark$ At the first stage, choose the first connected component according to some predefined order s.t. at least one agent voted for a location in this component.

$>$ At the second stage, run $\mathcal{F}^{\star}$ taking into account only ballots in the chosen component.

Note that, just like the mechanism for the connected case, also this mechanism can be equivalently defined as an order-based mechanism with the concatenation of the respective orders of the different components.

This scenario exemplifies again the high efficiency-cost of false-name-proofness. Since the graph is disconnected, it is unavoidable that some of the agents will have no access to the facility. Nevertheless, on efficiency grounds we would like to hurt the minimal number of agents. Requiring false-name-proofness, or equivalently assuming an agent can cast any number of ballots, means essentially that the designer must be oblivious to such arguments and act as-if any of the ballots might represent the vast majority of society. ${ }^{22}$

Recall that we saw in Claim 8 that there exist anonymous, Pareto-optimal, group-manipulation-resistant mechanisms for all connected graphs of up-to five locations. Hence, we get by the above insight anonymous, Pareto-optimal, group-manipulation-resistant mechanisms for all graphs of up-to five locations (both connected and disconnected). This is no longer true for larger graphs since we showed no such mechanism exists for the cycle of six locations.

For the scenario of an infinite number of agents, we get that $\mathcal{F}^{\star}$ still satisfies the desiderata (using the same proof).

For dealing with infinite graphs, we need to extend Definition 3 of simple $Z V$-line graphs and add a requirement that the linear order over the $Z$-locations is a well-order, that is, requiring that the leftmost location is defined for any (measurable) subset of $Z$-locations. Adding this assumption, our results extend (using the same proof) to show that $\mathcal{F}^{\star}$ satisfies the desiderata for infinite graphs as well (with either finite or infinite number of agents). Note that without the well-order assumption, $\mathcal{F}^{\star}$ might not be well-defined even when the number of

\footnotetext{
${ }^{22}$ Recall that by Proposition 1 bounding the number of false-ballots an agent might cast does not decrease the efficiency-loss.
} 


$$
V=\left\{v_{1}, v_{2}\right\} \quad ; \quad Z=\mathbb{Z} \quad ; \quad \mathcal{E}=V \times Z
$$

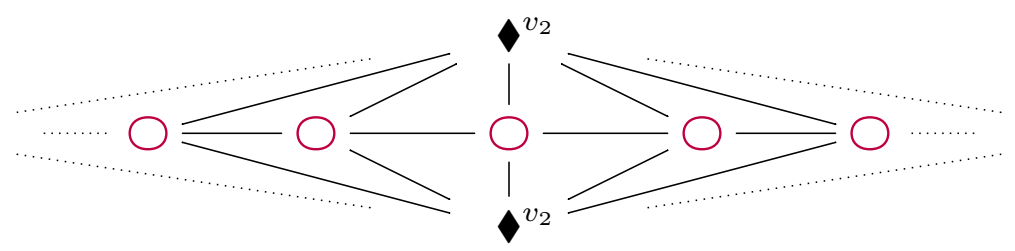

Fig. 20 Example: an infinite $Z V$-line graphs s.t. the leftmost Pareto-optimal $Z$-locations is not defined (Color figure online)

agents is finite. For instance, consider the infinite simple $Z V$-line graph in Fig. 20. Then, for the profile $\left\{v_{1}, v_{2}\right\}$ the leftmost Pareto-optimal $Z$-location is not defined.

\subsection{Approximate mechanism design without money}

Last, an important continuation of this work is analyzing the implications for approximate mechanism design without money [53]. That is, assuming the agents are quantitatively represented by a cost function (e.g., the distance to the facility or a monotone function of the distance) and analyzing the implications of manipulation-resistance on the approximability of the minimization problem of natural social cost functions. As we saw in Sect. 5.1, we expect to get a low performance guarantee for most natural social cost functions (of the same order of magnitude as the constant mechanism and the mechanism that outputs a random ballot). Weakening the false-name-proofness does not circumvent this bad efficiency phenomenon (by Proposition 1), and as we saw, this phenomenon is common to many other scenarios in which false-name-proofness is a desired property.

Nowadays, many aggregation mechanisms are highly susceptible to double-voting and false-name voting in general (e.g., mechanisms over massive anonymous networks like the internet and also other scenarios in which vote frauds are known to be easy). We think that such results should open a discussion on the costs of these protocols (since the benefits are clear) or direct us to look for solutions outside of the scope of mechanism design like those we surveyed in Sect. 5.1.

\section{Appendix A: Omitted proofs: Section 2 (Order-based mechanisms)}

Claim 3 There exists an anonymous, Pareto-optimal, group-manipulation-resistant mechanism $\mathcal{F}$ for the (2,3)-biclique s.t. $\mathcal{F}$ is not an order-based mechanism.

Proof Consider the following mechanism for the (2, 3)-biclique (see Fig. 21). 


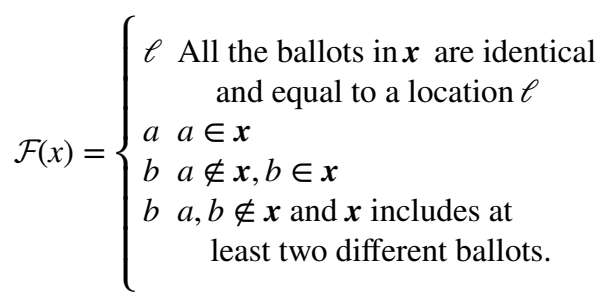

It is not hard to see that $\mathcal{F}$ is indeed an anonymous mechanism.

\section{$\mathcal{F}$ is a Pareto-optimal mechanism:}

In the first three cases, the outcome is equal to one of the ballots and hence, it is a Pareto-optimal outcome. In the fourth case, all the ballots belong to $\{u, v, w\}$ and include at least two different locations of $\{u, v, w\}$. The location $b$ is of distance one from all of the ballots. Any of the locations in $\{u, v, w\}$ is of distance two from at least one of the ballots, and the location $a$ is of distance one from all of the ballots. Hence, no location Pareto-dominates $b$ and $b$ is a Pareto-optimal outcome.

$\mathcal{F}$ is group-manipulation-resistant:

- In the first case, all agents received their best location, so no one would like to change the outcome.

- If the outcome is the location $a$, then necessarily one of the agents voted for $a$ and would not like to change the outcome. On the other hand, without the support of this agent, no coalition is able to change the outcome.

- Similarly, if $a \notin \boldsymbol{x}$ and $b \in \boldsymbol{x}$, then the outcome is $b$, the agent who voted for $b$ would not like to change the outcome, and without the support of this agent, no coalition can change the outcome.

- Last, if $a, b \notin x$ and there are at least two different ballots, then the only strict improvement for an agent is changing the outcome to be her own location $\ell \in\{u, v, w\}$. But for doing that she must get the support of all agents who did not vote for $\ell$ and in particular of the agent who voted for some $\ell^{\prime} \in\{u, v, w\} \backslash\{\ell\}$ who is strictly hurt by this change.

\section{$\mathcal{F}$ is not an order-based mechanism:}

Consider the three-ballot profile $\boldsymbol{x}=\langle u, v, w\rangle$ and the five-ballot profile $\boldsymbol{y}=\langle a, b, u, v, w\rangle$. Then, $P O(\boldsymbol{x})=P O(\boldsymbol{y})=\{a, b, u, v, w\}$ but $\mathcal{F}(\boldsymbol{x}) \neq \mathcal{F}(\boldsymbol{y})$. Hence, $\mathcal{F}$ cannot be defined as a function of the set of Pareto-optimal locations and in particular it is not an order-based mechanism.

Claim 4 There exists an anonymous, Pareto-optimal, group-manipulation-resistant mechanism $\mathcal{F}$ for the cycle of size four s.t. $\mathcal{F}$ is not invariant to ballot duplication.

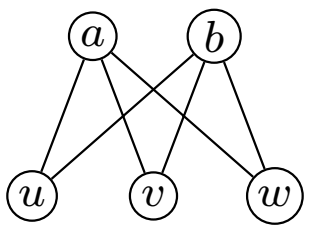

Fig. 21 The labeling of the (2,3)-biclique of the proof of Claim 3 


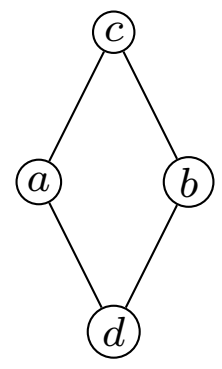

Fig. 22 The labeling of the $C_{4}$ of the proof of Claim 4

Proof Consider the following mechanism for the cycle of size four (see Fig. 22).

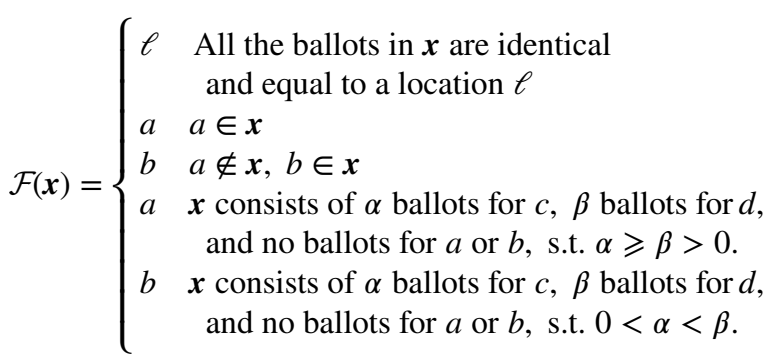

It is not hard to see that $\mathcal{F}$ is indeed an anonymous mechanism.

\section{$\mathcal{F}$ is a Pareto-optimal mechanism:}

In the first three cases, the outcome is equal to one of the ballots and hence it is a Paretooptimal outcome. In the fourth and fifth cases, all the ballots belong to $\{c, d\}$ and the profile includes both locations. Hence, both locations $a$ and $b$ are Pareto-optimal outcomes.

$\mathcal{F}$ is group-manipulation-resistant:

- In the first case, all agents received their best location, so no one would like to change the outcome.

- In the second case, the outcome is location $a$ and one of the agents voted for $a$ and would not like to change the outcome. On the other hand, without the support of this agent, no coalition is able to change the outcome.

- In the third case, the outcome is location $b$ and one of the agents voted for $b$ and would not like to change the outcome. On the other hand, without the support of this agent, a coalition can change the outcome only to be location $a$, which none of the agents strictly prefers over location $b$ (since no agent voted for $a$ ).

- In the fourth and fifth cases, all agents voted for either $c$ or $d$ so they are indifferent between the outcomes $a$ and $b$. On the other hand, a coalition can change the outcome to be location $c$ only if all of its members voted for location $d$, i.e., they strictly prefer not to change the outcome (and similarly for location $d$ ).

$\mathcal{F}$ is not invariant to duplicating ballots:

For example, consider the two-ballot profile $x=\langle c, d\rangle$ and the three-ballot profile $\boldsymbol{y}=\langle c, d, d\rangle$. Then, $\mathcal{F}(\boldsymbol{x})=a$ but $\mathcal{F}(\boldsymbol{y})=b$. 


\section{Appendix B: Omitted proof: Claim 8 (Graphs of at most five locations)}

In here, we show that all connected graphs with at most five locations except $C_{5}$, the cycle of five locations, are simple $Z V$-line graphs. As we saw in Sect. 6, there is an anonymous, Paretooptimal, group-manipulation-resistant mechanism for $C_{5}$. Therefore, we get that for all connected graphs with at most five locations there is an anonymous, Pareto-optimal, group-manipulation-resistant mechanism. In Sect. 7.1, we show that this fact gives us such mechanisms for disconnected graphs with at most five locations as well. Note this is no longer true for larger graphs since we showed no such mechanism exists for the cycle of six locations (Proposition 3).

The table below is a list of all connected graphs with at most five locations ${ }^{23}$ with their simple $Z V$-line graph representation, i.e., a partition to $V$-locations and $Z$-locations and an order over the latter (We use the figures $\bigcirc$ for $V$-locations and (2) for $Z$-locations with indices denoting the order over the $Z$-locations). For some of the graphs, there are also other mechanisms arising from other partitions, but we chose to show only one or two partitions to prove the graph is a simple $Z V$-line graph. For any of these graphs (except $C_{5}$ ) we are unaware of anonymous, Pareto-optimal, group-manipulation-resistant mechanisms that are not based on a representation of the graph as a simple $Z V$-line graph.

Connected graphs with two locations (Color figure online)

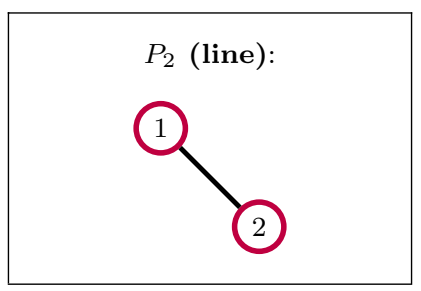

Connected graphs with three locations (2 graphs)(Color figure online)

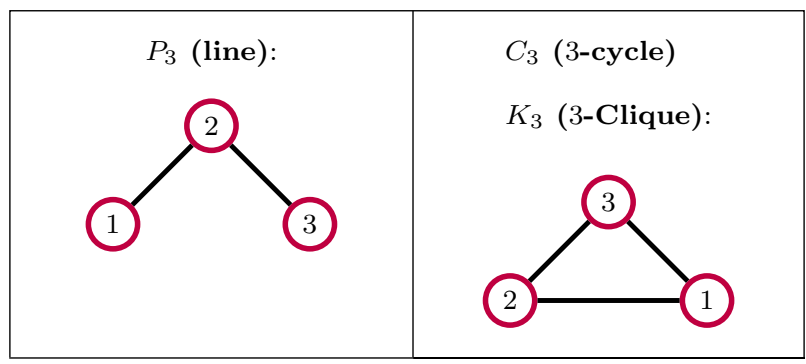

$\overline{{ }^{23}}$ We base the graph names on Information System on Graph Classes and their Inclusions (ISGCI) [17]. 
Connected graphs with four locations (6 graphs)(Color figure online)

1) $K_{4}(4$-Clique $) / W_{3}:$


Connected graphs with five locations (21 graphs) (Color figure online)

(1):




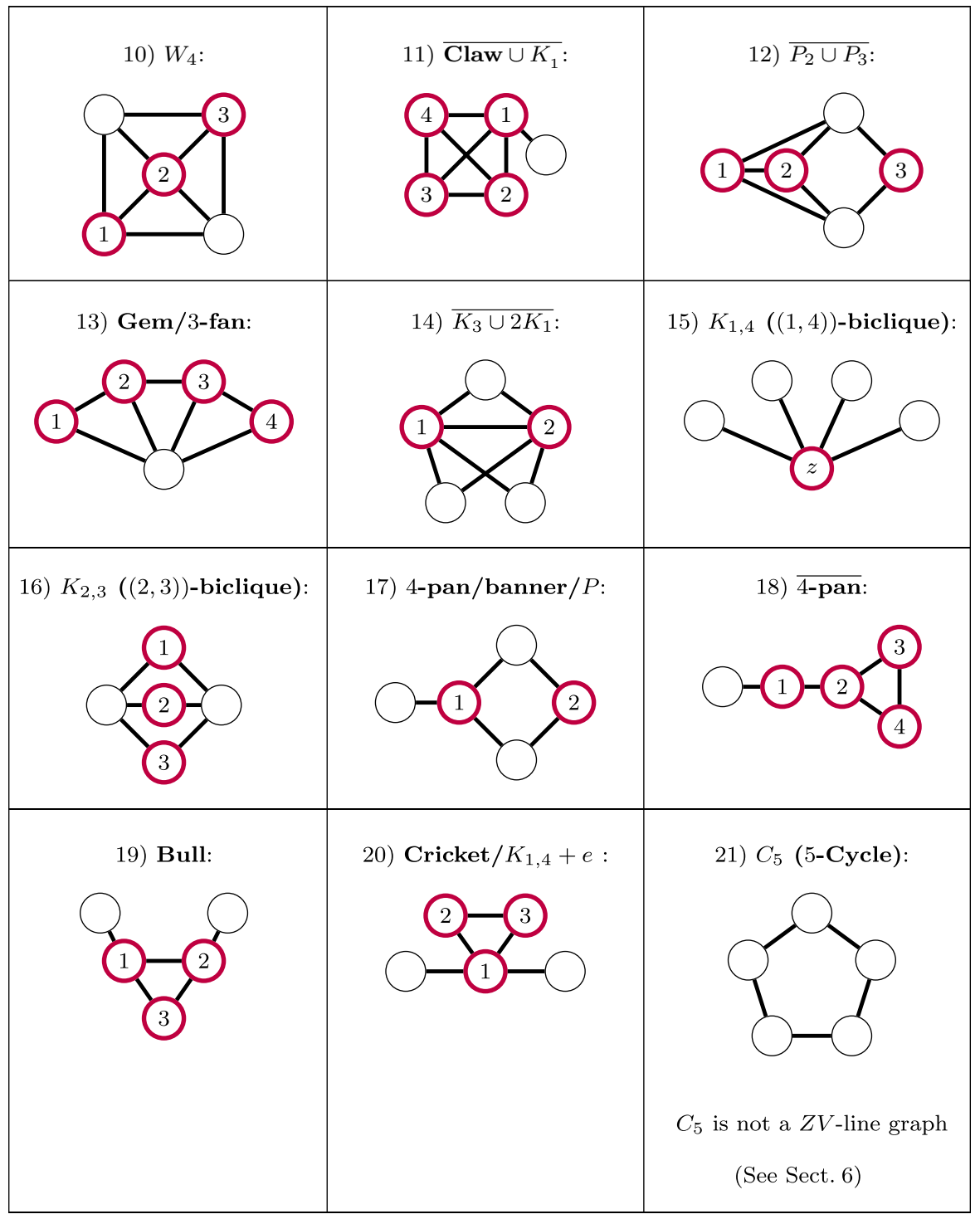

\section{Appendix C: Omitted proofs: Section 4 (ZV-line graphs)}

Lemma 1 For any location $\ell \in \mathcal{V}$, the preferences of an agent located on $\ell$ and an agent located on $\mathcal{R}(\ell)$ over the locations of $\mathcal{G}_{\perp}$ are identical. That is,

$$
\forall u, v \in V_{\perp}: \quad d(\ell, u) \leqslant d(\ell, v) \Longleftrightarrow d(\mathcal{R}(\ell), u) \leqslant d(\mathcal{R}(\ell), v) .
$$

Moreover, if $\ell \in V_{i}$ for some $i \in\{1, \ldots, k\}$, then the preferences of an agent located on $\ell$ and an agent located on $\mathcal{R}(\ell)$ over the locations outside of $V_{i} \backslash V_{\perp}$ are identical. That is, 


$$
\forall u, v \notin V_{i} \backslash V_{\perp}: \quad d(\ell, u) \leqslant d(\ell, v) \Longleftrightarrow d(\mathcal{R}(\ell), u) \leqslant d(\mathcal{R}(\ell), v) .
$$

Proof This lemma is trivial in case that $\ell=\mathcal{R}(\ell)$. Henceforth, we assume that $\ell \neq \mathcal{R}(\ell)$, i.e., $\ell \in V_{i} \backslash V_{\perp}$ for some $i \in\{1, \ldots, k\}$. W.1.o.g., $\ell \in V_{1} \backslash V_{\perp}=V_{1} \backslash\left\{\mathcal{R}\left(V_{1}\right)\right\}$.

By Definition 5, it is not hard to see that any path from $\ell$ to a location outside of $V_{i}$, $\ell^{\prime} \notin V_{i}$, must include $\mathcal{R}\left(\mathcal{G}_{i}\right)=\mathcal{R}(\ell)$, and hence,

$$
d\left(\ell, \ell^{\prime}\right)=d(\ell, \mathcal{R}(\ell))+d\left(\mathcal{R}(\ell), \ell^{\prime}\right)
$$

(Note that the above equality holds also for $\ell^{\prime}=\mathcal{R}(\ell)$ ). Therefore, for any two locations $u, v \notin V_{i} \backslash V_{\perp}$,

$$
\begin{aligned}
d(\ell, v)-d(\ell, u) & =[d(\ell, \mathcal{R}(\ell))+d(\mathcal{R}(\ell), v)]-[d(\ell, \mathcal{R}(\ell))+d(\mathcal{R}(\ell), u)] \\
& =d(\mathcal{R}(\ell), v)-d(\mathcal{R}(\ell), u) .
\end{aligned}
$$

Proposition 7 Let $\mathcal{G}=\langle\mathcal{V}, \mathcal{E}\rangle$ be an unweighted undirected connected graph with a nontrivial cover $V_{\perp}, V_{1}, \cdots, V_{k} \quad(k \geqslant 1)$, and denote by $\mathcal{G}_{i}$ the subgraph $\left\langle\mathcal{V}=V_{i}, \mathcal{E}=\mathcal{E} \cap\left(\begin{array}{c}V_{i} \\ 2\end{array}\right)\right\rangle$, s.t.

- For $i=1, \ldots, k$, there exists a unique location in $V_{i} \cap V_{\perp}$, which we denote by $\mathcal{R}\left(\mathcal{G}_{i}\right)$.

- For $i=1, \ldots, k$, there are no edges between locations in $\left(V_{i} \backslash\left\{\mathcal{R}\left(\mathcal{G}_{i}\right)\right\}\right)$ and locations outside of $V_{i}$. (Equivalently, all paths between locations of $V_{i}$ and locations outside of $V_{i}$ include the root $\mathcal{R}\left(\mathcal{G}_{i}\right)$ ).

- The sets $\left\{V_{i} \backslash\left\{\mathcal{R}\left(\mathcal{G}_{i}\right)\right\}\right\}_{i=1, \ldots, k}$ are pairwise disjoint.

For $i=\perp, 1, \ldots, k$, let $\mathcal{F}_{i}: \boldsymbol{x} \in V_{i}^{\star} \rightarrow V_{i}$ be a mechanism for $\mathcal{G}_{i}$ s.t.

- $\mathcal{F}_{i}$ is an anonymous, Pareto-optimal, group-manipulation-resistant mechanism.

- For an infinite number of $\tau \in \mathbb{N}$, there exists a profile $\boldsymbol{x} \in V_{i}^{\star}$ in which there are at least $\tau$ ballots for any location in $V_{i}$ s.t. $\mathcal{F}_{i}(\boldsymbol{x})=\mathcal{R}\left(\mathcal{G}_{i}\right)$.

Then, for $\mathcal{F}_{\text {rec }}^{\star}: \mathcal{V}^{\star} \rightarrow \mathcal{V}$ as defined in Definition 6 ,

- $\mathcal{F}_{\text {rec }}^{\star}$ is an anonymous, Pareto-optimal, group-manipulation-resistant mechanism.

- For any number $\tau \in \mathbb{N}$, there exists a profile $\boldsymbol{x} \in \mathcal{V}^{\star}$ in which there are at least $\tau$ ballots for any location in $\mathcal{V}$ s.t. $\mathcal{F}_{\text {rec }}^{\star}(\boldsymbol{x})=\mathcal{R}(\mathcal{G})=\mathcal{R}\left(\mathcal{G}_{\perp}\right)$.

We prove a more robust version of Proposition 7 showing that also when the mechanisms $\mathcal{F}_{i}$ satisfy a subset of the desired properties, then also $\mathcal{F}_{\text {rec }}^{\star}$ satisfies this subset.

Proposition 12 Let $\mathcal{G}=\langle\mathcal{V}, \mathcal{E}\rangle$ be an unweighted undirected connected graph with a nontrivial cover $V_{\perp}, V_{1}, \cdots, V_{k} \quad(k \geqslant 1)$, and denote by $\mathcal{G}_{i}$ the subgraph $\left\langle\mathcal{V}=V_{i}, \mathcal{E}=\mathcal{E} \cap\left(\begin{array}{c}V_{i} \\ 2\end{array}\right)\right\rangle$, s.t. 
- For $i=1, \ldots, k$, there exists a unique location in $V_{i} \cap V_{\perp}$, which we denote by $\mathcal{R}\left(\mathcal{G}_{i}\right)$.

- $\quad$ For $i=1, \ldots, k$, there are no edges between locations in $\left(V_{i} \backslash\left\{\mathcal{R}\left(\mathcal{G}_{i}\right)\right\}\right)$ and locations outside of $V_{i}$. (Equivalently, all paths between locations of $V_{i}$ and locations outside of $V_{i}$ include the root $\mathcal{R}\left(\mathcal{G}_{i}\right)$ ).

- The sets $\left\{V_{i} \backslash\left\{\mathcal{R}\left(\mathcal{G}_{i}\right)\right\}\right\}_{i=1, \ldots, k}$ are pairwise disjoint.

For $i=\perp, 1, \ldots, k$, let $\mathcal{F}_{i}: x \in V_{i}^{\star} \rightarrow V_{i}$ be a mechanism for $\mathcal{G}_{i}$ s.t.

- $\mathcal{F}_{i}$ is an anonymous Pareto-optimal mechanism.

- $\mathcal{F}_{i}$ is resistant against false-name-voting of any single agent.

- For an infinite number of $\tau \in \mathbb{N}$, there exists a profile $\boldsymbol{x} \in V_{i}^{\star}$ in which there are at least $\tau$ ballots for any location in $V_{i}$ s.t. $\mathcal{F}_{i}(\boldsymbol{x})=\mathcal{R}\left(\mathcal{G}_{i}\right)$.

Then, for $\mathcal{F}_{\text {rec }}^{\star}: \mathcal{V}^{\star} \rightarrow \mathcal{V}$ as defined in Definition 6 ,

- $\mathcal{F}_{\text {rec }}^{\star}$ is an anonymous Pareto-optimal mechanism.

- For any number $\tau \in \mathbb{N}$, there exists a profile $\boldsymbol{x} \in \mathcal{V}^{\star}$ in which there are at least $\tau$ ballots for any location in $\mathcal{V}$ s.t. $\mathcal{F}_{\text {rec }}^{\star}(\boldsymbol{x})=\mathcal{R}(\mathcal{G})=\mathcal{R}\left(\mathcal{G}_{\perp}\right)$.

- For any profile of locations $\boldsymbol{x} \in \mathcal{V}^{\star}$, a coalition of agents $C$, and a set of ballots $\boldsymbol{A} \in \mathcal{V}^{\star}$ s.t. $\boldsymbol{A}$ is a beneficial deviation for $C$,

- If there exists $t \in\{1, \ldots, k\}$ s.t either all the ballots in $\boldsymbol{x}$ or all the ballots of $\left\langle\boldsymbol{A}, \boldsymbol{x}_{-C}\right\rangle$ are locations of $\mathcal{G}_{t}$, then $\boldsymbol{A}$ is a beneficial manipulation of $\mathcal{F}_{t}$ for the coalition $C$ in the profile $\boldsymbol{x}$.

- Otherwise, $\mathcal{R}(\boldsymbol{A})$ is a beneficial manipulation of $\mathcal{F}_{\perp}$ for the coalition $C$ in the profile $\mathcal{R}(\boldsymbol{x})$.

Proof of Proposition 12 Since the mechanisms $\mathcal{F}_{i}$ for $i=\perp, 1, \ldots, k$ are all anonymous mechanisms, then also $\mathcal{F}_{\text {rec }}^{\star}$ is an anonymous mechanism. It is also not hard to see that $\mathcal{F}_{\text {rec }}^{\star}(\boldsymbol{x}) \in P O(\boldsymbol{x})$ for any location profile $\boldsymbol{x} \in \mathcal{V}^{\star}$ :

- If there exists a subgraph $\mathcal{G}_{t}$ for some $t \in\{1, \ldots, k\}$ s.t. all ballots in $\boldsymbol{x}$ belong to $V_{t}$, then, by Corollary $1, \mathcal{R}\left(\mathcal{G}_{t}\right)$ Pareto-dominates any location outside of $V_{t}$, so $P O(x) \subseteq V_{t}$. Furthermore, any location $\ell \in V_{t} \backslash P O(x)$ is Pareto-dominated by a location $\ell^{\prime} \in P O(x) \subseteq V_{t}$. Hence, the Pareto-optimal set when considering only the locations in $V_{t}$ is equal to the Pareto-optimal set when considering all locations in $\mathcal{V}$. Since $\mathcal{F}_{t}$ is a Pareto-optimal mechanism, we get that

$$
\mathcal{F}_{\text {rec }}^{\star}(\boldsymbol{x})=\mathcal{F}_{t}(\boldsymbol{x}) \in P O(\boldsymbol{x}) .
$$

- Otherwise, $\mathcal{F}_{\text {rec }}^{\star}(\boldsymbol{x})=\mathcal{F}_{\perp}(\mathcal{R}(\boldsymbol{x})) \in V_{\perp}$. Assume for contradiction that there exists a location $\ell$ Pareto-dominating $\mathcal{F}_{\text {rec }}^{\star}(\boldsymbol{x})$.

- If $\ell \in V_{\perp}$, then by Lemma $1, \ell$ Pareto-dominates $\mathcal{F}_{\text {rec }}^{\star}(\boldsymbol{x})=\mathcal{F}_{\perp}(\mathcal{R}(\boldsymbol{x}))$ for the profile $\mathcal{R}(\boldsymbol{x})$, in contradiction to the Pareto-optimality of $\mathcal{F}_{\perp}$.

- Otherwise, $\ell \in V_{t} \backslash V_{\perp}$ for some $t \in\{1, \ldots, k\}$ and we claim that $\mathcal{R}(\ell)$ that Pareto-dominates $\mathcal{F}_{\text {rec }}^{\star}(\boldsymbol{x})=\mathcal{F}_{\perp}(\mathcal{R}(\boldsymbol{x}))$ for the profile $\mathcal{R}(\boldsymbol{x})$, in contradiction to the Pareto-optimality of $\mathcal{F}_{\perp}$. 
- If Agent $i$ is located in $V_{t}$, then $\mathcal{R}(\ell)=\mathcal{R}\left(x_{i}\right)=\mathcal{R}\left(V_{t}\right)$ and hence an agent located on $\mathcal{R}\left(x_{i}\right)$ weakly prefers $\mathcal{R}(\ell)$ over $\mathcal{F}_{\text {rec }}^{\star}(\boldsymbol{x})$.

- If Agent $i$ is located outside of $V_{t}$ (and note that necessarily there exists at least one), then Agent $i$ strictly prefers $\mathcal{R}\left(V_{t}\right)=\mathcal{R}(\ell)$ over $\ell$ and since $\ell$ Pareto-dominates $\mathcal{F}_{\text {rec }}^{\star}(\boldsymbol{x})$ for the profile $\boldsymbol{x}$, Agent $i$ also strictly prefers $\mathcal{R}(\ell)$ over $\mathcal{F}_{\text {rec }}^{\star}(\boldsymbol{x})$. By Lemma 1 , an agent located on $\mathcal{R}\left(x_{i}\right)$ strictly prefers $\mathcal{R}(\ell)$ over $\mathcal{F}_{\text {rec }}^{\star}(\boldsymbol{x})$.

Let $\tau \in \mathbb{N}$. We will construct a location profile $\boldsymbol{x} \in \mathcal{V}^{\star}$ in which there are at least $\tau$ ballots for any location in $\mathcal{V}$ and $\mathcal{F}_{\text {rec }}^{\star}(\boldsymbol{x})=\mathcal{R}(\mathcal{G})$.

Let $\widehat{\boldsymbol{x}^{\mathbf{1}}} \in \mathcal{V}^{\star}$ be a profile in which there are at least $\tau$ ballots for any location in $\mathcal{V}$. We note that in $\mathcal{R}\left(\widehat{\boldsymbol{x}^{\mathbf{1}}}\right)$, there are ballots only for locations in $V_{\perp}$, and define $\rho$ to be the maximal number of ballots in $\mathcal{R}\left(\widehat{\boldsymbol{x}^{\mathbf{1}}}\right)$ for a location in $V_{\perp}$. We are given that there exists a profile $\widehat{\boldsymbol{x}^{2}} \in\left(V_{\perp}\right)^{\star}$ in which there are at least $\rho$ ballots for any location in $V_{\perp}$ and $\mathcal{F}_{\perp}\left(\widehat{\boldsymbol{x}^{\mathbf{2}}}\right)=\mathcal{R}\left(\mathcal{G}_{\perp}\right)$. We define the profile $\boldsymbol{x} \in \mathcal{V}^{\star}$ by adding ballots to $\widehat{\boldsymbol{x}^{\mathbf{1}}}$ as follows: For any $\ell \in V_{\perp}$, we add the difference between the number of ballots for $\ell$ in $\widehat{x^{2}}$ and the number of ballots for $\ell$ in $\mathcal{R}\left(\widehat{x}^{\mathbf{1}}\right)$.

Then, for any location $\ell \in \mathcal{V}$, there are at least $\tau$ ballots in $\boldsymbol{x}$, and $\mathcal{R}(\boldsymbol{x})=\widehat{\boldsymbol{x}^{2}}$. Since $V_{i} \nsubseteq V_{\perp}$ for all $i \in\{1, \ldots, k\}$, and since $\mathcal{R}(\ell)=\ell$ for any location $\ell \in V_{\perp}$ we get

$$
\mathcal{F}_{\text {rec }}^{\star}(\boldsymbol{x})=\mathcal{F}_{\perp}(\mathcal{R}(\boldsymbol{x}))=\mathcal{F}_{\perp}\left(\widehat{\boldsymbol{x}^{\mathbf{2}}}\right)=\mathcal{R}\left(\mathcal{G}_{\perp}\right)=\mathcal{R}(\mathcal{G})
$$

Last, we prove the main part of the proposition dealing the manipulation-resistance properties of $\mathcal{F}_{\text {rec }}^{\star}$. Let $\boldsymbol{x} \in \mathcal{V}^{\star}$ be a location profile, $C$ a coalition of agents, and $\boldsymbol{A} \in \mathcal{V}^{\star}$ a set of ballots s.t. $\boldsymbol{A}$ the coalition $C$ can cast $\boldsymbol{A}$ and change the outcome to be $\mathcal{F}_{\text {rec }}^{\star}\left(\boldsymbol{A}, \boldsymbol{x}_{-C}\right)$ that it strictly prefers. That is, all the agents in $C$ weakly prefer $\mathcal{F}_{\text {rec }}^{\star}\left(\boldsymbol{A}, \boldsymbol{x}_{-C}\right)$ over $\mathcal{F}_{\text {rec }}^{\star}(\boldsymbol{x})=\mathcal{F}_{\text {rec }}^{\star}\left(\boldsymbol{x}_{C}, \boldsymbol{x}_{-C}\right)$ and at least one agent in $C$, Agent $i$ for $i \in C$, strictly prefers $\mathcal{F}_{\text {rec }}^{\star}\left(\boldsymbol{A}, \boldsymbol{x}_{-C}\right)$ over $\mathcal{F}_{\text {rec }}^{\star}(\boldsymbol{x}) . \mathcal{F}_{\text {rec }}^{\star}(\boldsymbol{x}) \in P O(\boldsymbol{x})$ and in particular the coalition of all agents does not strictly prefer $\mathcal{F}_{\text {rec }}^{\star}\left(\boldsymbol{A}, \boldsymbol{x}_{-C}\right)$ over $\mathcal{F}_{\text {rec }}^{\star}(\boldsymbol{x})$. Hence, there exists an Agent $j$, for $j \notin C$, who strictly prefers $\mathcal{F}_{\text {rec }}^{\star}(\boldsymbol{x})$ over $\mathcal{F}_{\text {rec }}^{\star}\left(\boldsymbol{A}, \boldsymbol{x}_{-C}\right)$.

Case (I): There exists $t \in\{1, \ldots, k\}$ s.t. all the ballots in $x$ are locations of $\mathcal{G}_{t^{*}}$.

Then by the definition of $\mathcal{F}_{\text {rec }}^{\star}, \mathcal{F}_{\text {rec }}^{\star}(\boldsymbol{x})=\mathcal{F}_{i}(\boldsymbol{x})$. Agent $i$ can change the outcome of $\mathcal{F}_{t}$ to be $\mathcal{R}\left(\mathcal{G}_{t}\right)$ by casting enough false-name ballots. Since $\mathcal{F}_{t}$ is resistant to false-name manipulations of Agent $i$, we get that Agent $i$ weakly prefers $\mathcal{F}_{\text {rec }}^{\star}(\boldsymbol{x})$ over $\mathcal{R}\left(\mathcal{G}_{t}\right)$.

By Corollary 1 , Agent $i$ strictly prefers $\mathcal{R}\left(\mathcal{G}_{t}\right)$ over any location outside of $\mathcal{G}_{t}$. Hence, Agent $i$ strictly prefers $\mathcal{F}_{\text {rec }}^{\star}\left(\boldsymbol{A}, \boldsymbol{x}_{-C}\right)$ over $\mathcal{F}_{\text {rec }}^{\star}(\boldsymbol{x}), \mathcal{R}\left(\mathcal{G}_{t}\right)$, and any location outside of $\mathcal{G}_{t}$. In particular,

$$
\mathcal{F}_{\text {rec }}^{\star}\left(\boldsymbol{A}, \boldsymbol{x}_{-C}\right) \in V_{t} \backslash\left\{\mathcal{F}_{\text {rec }}^{\star}(\boldsymbol{x}), \mathcal{R}\left(\mathcal{G}_{t}\right)\right\} \subseteq V_{t} \backslash V_{\perp} .
$$

Hence, by the definition of $\mathcal{F}_{\text {rec }}^{\star}$, necessarily $\boldsymbol{A} \subseteq V_{t}$ and $\mathcal{F}_{\text {rec }}^{\star}\left(\boldsymbol{A}, \boldsymbol{x}_{-C}\right)=\mathcal{F}_{t}\left(\boldsymbol{A}, \boldsymbol{x}_{-C}\right)$. So $\boldsymbol{A}$ is a beneficial manipulation of $\mathcal{F}_{t}$ for $C$ in $\boldsymbol{x}$.

Case (II): There exists $t \in\{1, \ldots, k\}$ s.t. all the ballots in $\left\langle\boldsymbol{A}, \boldsymbol{x}_{-C}\right\rangle$ are locations of $\mathcal{G}_{t}$.

Then by the definition of $\mathcal{F}_{\text {rec }}^{\star}, \mathcal{F}_{\text {rec }}^{\star}\left(\boldsymbol{A}, \boldsymbol{x}_{-C}\right)=\mathcal{F}_{i}\left(\boldsymbol{A}, \boldsymbol{x}_{-C}\right)$. Agent $j$ can change the outcome of $\mathcal{F}_{t}$ to be $\mathcal{R}\left(\mathcal{G}_{t}\right)$ by casting enough false-name ballots. Since $\mathcal{F}_{t}$ is resistant to 
false-name manipulations of Agent $j$, we get that Agent $j$ weakly prefers $\mathcal{F}_{\text {rec }}^{\star}\left(\boldsymbol{A}, \boldsymbol{x}_{-C}\right)$ over $\mathcal{R}\left(\mathcal{G}_{t}\right)$.

By Corollary 1 , Agent $j$ strictly prefers $\mathcal{R}\left(\mathcal{G}_{t}\right)$ over any location outside of $\mathcal{G}_{t}$. Hence, Agent $j$ strictly prefers $\mathcal{F}_{\text {rec }}^{\star}(\boldsymbol{x})$ over $\mathcal{F}_{\text {rec }}^{\star}\left(\boldsymbol{A}, \boldsymbol{x}_{-C}\right), \mathcal{R}\left(\mathcal{G}_{t}\right)$, and any location outside of $\mathcal{G}_{t}$. In particular,

$$
\mathcal{F}_{\text {rec }}^{\star}(\boldsymbol{x}) \in V_{t} \backslash\left\{\mathcal{F}_{\text {rec }}^{\star}(\boldsymbol{x}), \mathcal{R}\left(\mathcal{G}_{t}\right)\right\} \subseteq V_{t} \backslash V_{\perp} .
$$

Hence, by the definition of $\mathcal{F}_{\text {rec }}^{\star}$, necessarily all the ballots in $x$ are locations of $\mathcal{G}_{t}$ and $\mathcal{F}_{\text {rec }}^{\star}(\boldsymbol{x})=\mathcal{F}_{t}(\boldsymbol{x})$. So $\boldsymbol{A}$ is a beneficial manipulation of $\mathcal{F}_{t}$ for $C$ in $\boldsymbol{x}$.

Case (III): Neither of the previous two cases holds.

Then,

$$
\left\{\begin{array}{l}
\mathcal{F}_{\text {rec }}^{\star}(\boldsymbol{x})=\mathcal{F}_{\perp}(\mathcal{R}(\boldsymbol{x})) \in V_{\perp} \\
\mathcal{F}_{\text {rec }}^{\star}\left(\boldsymbol{A}, \boldsymbol{x}_{-C}\right)=\mathcal{F}_{\perp}\left(\mathcal{R}(\boldsymbol{A}), \mathcal{R}\left(\boldsymbol{x}_{-C}\right)\right) \in V_{\perp},
\end{array}\right.
$$

and by Lemma 1 we get that $\mathcal{R}(\boldsymbol{A})$ is a beneficial manipulation of $\mathcal{F}_{\perp}$ for $C$ in $\mathcal{R}(\boldsymbol{x})$.

\section{Appendix D: Omitted proofs: Section 6 (The discrete cycle over $n$ locations)}

\section{Proposition 8}

- The only false-name-proof, anonymous, Pareto-optimal mechanisms for $C_{2}$ are the two order-based mechanisms.

- The only false-name-proof, anonymous, Pareto-optimal mechanisms for $C_{3}$ are the six order-based mechanisms.

The proofs for the two graphs are almost identical. Still, we think it would be easier to follow if we prove them separately.

Proof $\left(\boldsymbol{C}_{2}\right)$ First, we note that for any location profile $\boldsymbol{x}$ the set of Pareto-optimal locations $P O(x)$ is equal to the set of locations that were voted for. Let $\mathcal{F}$ be a false-name-proof, anonymous, Pareto-optimal mechanism for $C_{2}$. Clearly, when all ballots are identical and equal to a location $\ell$, by Pareto-optimality the outcome must be $\ell$. Assume there exist two location profiles $\boldsymbol{x}$ and $\boldsymbol{y}$ that include both locations s.t. $\mathcal{F}(\boldsymbol{x}) \neq \mathcal{F}(\boldsymbol{y})$. Consider the union profile $z=\boldsymbol{x} \cup \boldsymbol{y}$ and w.l.o.g. assume that $\mathcal{F}(z) \neq \mathcal{F}(\boldsymbol{x})$. Then, the agent who voted for $\mathcal{F}(z)$ in $x$ can add false-name ballots and change the outcome to be $\mathcal{F}(z)$ which she strictly prefers over $\mathcal{F}(\boldsymbol{x})$. Hence, $\mathcal{F}$ can be defined via a selection function from the set of Paretooptimal locations.

Consider the location profile $\boldsymbol{x}$ in which all locations were voted for exactly once, and denote the other location besides $\mathcal{F}(\boldsymbol{x})$ by $b$. Then, whenever there are ballots for both locations the outcome must be $\mathcal{F}(\boldsymbol{x})$, and by false-name-proofness, whenever one of the agents casts a ballot for the location $\mathcal{F}(\boldsymbol{x})$ the outcome must be $\mathcal{F}(\boldsymbol{x})$.

Hence, $\mathcal{F}$ is the order-based mechanism defined by $\mathcal{F}(\boldsymbol{x}) \rightarrow b$.

Proof $\left(\boldsymbol{C}_{3}\right)$ First, we note that for any location profile $\boldsymbol{x}$ the set of Pareto-optimal locations $P O(x)$ is equal to the set of locations that were voted for. Let $\mathcal{F}$ be a false-name-proof, 
anonymous, Pareto-optimal mechanism for $C_{3}$. Clearly, when all ballots are identical and equal to a location $\ell$, by Pareto-optimality the outcome must be $\ell$.

Assume there exist two location profiles $\boldsymbol{x}$ and $\boldsymbol{y}$ that include the same set of locations s.t. $\mathcal{F}(\boldsymbol{x}) \neq \mathcal{F}(\boldsymbol{y})$. Consider the union profile $z=\boldsymbol{x} \cup \boldsymbol{y}$ and w.l.o.g. assume that $\mathcal{F}(\boldsymbol{z}) \neq \mathcal{F}(\boldsymbol{x})$. Then, the agent who voted for $\mathcal{F}(z)$ in $\boldsymbol{x}$ can add false-name ballots and change the outcome to be $\mathcal{F}(z)$ which she strictly prefers over $\mathcal{F}(x)$. Hence, $\mathcal{F}$ can be defined via a selection function from the set of Pareto-optimal locations.

Consider the location profile $\boldsymbol{x}$ in which all locations were voted for exactly once Then, whenever there are ballots for all locations the outcome must be $\mathcal{F}(\boldsymbol{x})$, and by false-nameproofness, whenever one of the agents casts a ballot for the location $\mathcal{F}(\boldsymbol{x})$ the outcome must be $\mathcal{F}(\boldsymbol{x})$. Denote the other two locations of the graph besides $\mathcal{F}(\boldsymbol{x})$ by $b$ and $c$ s.t. $\mathcal{F}(\langle b, c\rangle)=b$.

Then, $\mathcal{F}$ is the order-based mechanism defined by $\mathcal{F}(\boldsymbol{x}) \rightarrow b \rightarrow c$.

Proposition $9 \mathcal{F}$ is a false-name-proof, anonymous, Pareto-optimal mechanism for $C_{4}$ iff there exists a cyclic labeling of the graph by $\{a, b, c, d\}$ (see Fig. 17) s.t. for any profile $x \in \mathcal{V}^{\star}$

- If all ballots in $\boldsymbol{x}$ are equal to the same location $\ell$, then $\mathcal{F}(\boldsymbol{x})=\ell$.

- If $a \in \boldsymbol{x}$, then $\mathcal{F}(\boldsymbol{x})=a$.

- If $a \notin \boldsymbol{x}$ and $c \in \boldsymbol{x}$, then $\mathcal{F}(\boldsymbol{x})=c$.

- If $\boldsymbol{x}$ includes ballots for both location $b$ and location $d$ and only these two locations, then $\mathcal{F}(\boldsymbol{x}) \in\{a, c\}$.

Proof It is not hard to see that indeed all these mechanisms are false-name-proof, anonymous, Pareto-optimal mechanisms and actually group-manipulation-resistant mechanisms. We will show that these are the only false-name-proof, anonymous, Pareto-optimal mechanisms.

Let $\mathcal{F}$ be a false-name-proof, anonymous, Pareto-optimal mechanism for $C_{4}$. We denote the locations of $C_{4}$ by $\{a, b, c, d\}$ s.t. for an infinite number of profiles $\boldsymbol{x} \in \mathcal{V}^{\star}$ in which there is the same number of ballots for all four locations $\mathcal{F}(\boldsymbol{x})=a$.

From Pareto-optimality of $\mathcal{F}$ we get that whenever all ballots are identical and equal to a location $\ell, \mathcal{F}(\boldsymbol{x})=\ell$. Since $\mathcal{F}$ is a false-name-proof mechanism and since any agent can change the outcome to be location $a$ by casting enough false-name ballots, we get that

- Whenever one of the agents casts a ballot for location $a$, location $a$ must be the outcome, otherwise this agent would have a false-name manipulation.

- Whenever one of the agents casts a ballot for location $b$, the outcome cannot be location $d$, otherwise this agent would have a false-name manipulation to change the outcome to location $a$.

Similarly, whenever one of the agents casts a ballot for location $d$, the outcome cannot be location $b$.

In particular, when $\boldsymbol{x}$ includes ballots for both location $b$ and location $d$, then $\mathcal{F}(\boldsymbol{x}) \in\{a, c\}$.

- Last, we note that for any profile $\boldsymbol{x}$ which includes ballots for location $b$, location $c$, and location $d$ (but not location $a$ ): Location $a$ is Pareto-dominated by location $c$ and hence the outcome must be location $c$. 
Moreover, for any sub-profile of such a profile that includes a ballot for location $c$ : Location $c$ must be the outcome, otherwise the agent who voted for location $c$ would have a false-name manipulation to change the outcome to location $c$.

Proposition 10 The only false-name-proof, anonymous, Pareto-optimal mechanisms for $C_{5}$ are the following 30 order-based mechanisms (see Fig. 18):

- $a \rightarrow b \rightarrow e \rightarrow c \rightarrow d$,

- $a \rightarrow b \rightarrow e \rightarrow d \rightarrow c$,

- $a \rightarrow b \rightarrow d \rightarrow e \rightarrow c$,

- and their rotations and reflections.

Proof It is not hard to see that indeed all these mechanisms are false-name-proof, anonymous, Pareto-optimal mechanisms and actually group-manipulation-resistant mechanisms. We will show that these are the only false-name-proof, anonymous, Pareto-optimal mechanisms.

Let $\mathcal{F}$ be a false-name-proof, anonymous, Pareto-optimal mechanism for $C_{5}$. Necessarily, there exists a location $\ell$ s.t. for an infinite number of profiles $x \in \mathcal{V}^{\star}$ in which there is the same number of ballots for all five locations $\mathcal{F}(\boldsymbol{x})=\ell$. We denote this location by $a$ and the other four location by $\{b, c, d, e\}$ in a circular order (either clock-wise or counter clock-wise).

From Pareto-optimality of $\mathcal{F}$ we get that whenever all ballots are identical and equal to a location $\ell, \mathcal{F}(\boldsymbol{x})=\ell$. Since $\mathcal{F}$ is a false-name-proof mechanism and since any agent can change the outcome to be location $a$ by casting enough false-name ballots, we get that

- Whenever one of the agents casts a ballot for location $a$, location $a$ must be the outcome, otherwise this agent would have a false-name manipulation.

- When there are ballots for both location $b$ and location $e$, the outcome must be location $a$, otherwise at least one of these agents would have a false-name manipulation to change the outcome to be $a$.

Next, consider the 3-ballot profile $\langle b, c, d\rangle$. Location $a$ is Pareto-dominated by location $c$, so by false-name-proofness to manipulations of the first agent, the outcome must be either location $b$ or location $c$. Similarly, $\mathcal{F}(\langle c, d, e\rangle) \in\{d, e\}$.

Case (I): $\left\{\begin{array}{l}\mathcal{F}(\langle b, c, d\rangle)=b \\ \mathcal{F}(\langle c, d, e\rangle)=e\end{array}\right.$

By false-name-proofness, whenever there are ballots for location $b$, location $c$, and location $d$ (and maybe other locations as well), the outcome cannot be location $c$, otherwise the second agent in the profile $\langle b, c, d\rangle$ would have a false-name manipulation, and it cannot be location $d$, otherwise the third agent in the profile $\langle b, c, d\rangle$ would have a false-name manipulation. Similarly, the outcome when there are ballots for location $c$, location $d$, and location $e$ (and maybe other locations as well) cannot be location $d$ or location $e$. Next, we note that by false-name-proofness, 
- Whenever there are ballots only for location $b$ and location $c$, the outcome must be location $b$, since the agent who voted for it can change the outcome to be location $b$ by casting enough false-name ballots.

and similarly

- Whenever there are ballots only for locations $b$ and $d$, the outcome must be location $b$.

- Whenever there are ballots only for locations $c$ and $e$, the outcome must be location $e$.

- Whenever there are ballots only for locations $d$ and $e$, the outcome must be location $e$.

Last, we claim that for all profiles in which there are ballots only for location $d$ and location $e$, the outcome must be the same. Assume towards a contradiction that there exist two location profiles $\boldsymbol{x}$ and $\boldsymbol{y}$ that include only these two locations s.t. $\mathcal{F}(\boldsymbol{x}) \neq \mathcal{F}(\boldsymbol{y})$. By Paretooptimality the outcome of both must be either location $c$ or location $d$. Consider the union profile $z=\boldsymbol{x} \cup \boldsymbol{y}$ and w.l.o.g. assume that $\mathcal{F}(\boldsymbol{x}) \neq \mathcal{F}(z)$. Then, the agent who voted for $\mathcal{F}(z)$ in $x$ can add false-name ballots and change the outcome to be $\mathcal{F}(z)$ which she strictly prefers over $\mathcal{F}(\boldsymbol{x})$.

Hence, we get the first two order-based mechanisms in the proposition, which differ only in the outcome for profiles in which there are ballots only for location $d$ and location $e$.

Case (II): $\left\{\begin{array}{l}\mathcal{F}(\langle b, c, d\rangle)=c \\ \mathcal{F}(\langle c, d, e\rangle)=d\end{array}\right.$

Consider the 2-ballot profile $\langle c, d\rangle$. Both agents can get the outcome to be of distance 0 from their locations by casting one false-name ballot. Hence, for any possible outcome $\mathcal{F}(\langle c, d\rangle)$ at lease one of them would have a false-name manipulation, so we get a contradiction.

$$
\text { Case (III): }\left\{\begin{array}{l}
\mathcal{F}(\langle b, c, d\rangle)=b \\
\mathcal{F}(\langle c, d, e\rangle)=d
\end{array} \text { (and symmetrically Case (IV): }\left\{\begin{array}{l}
\mathcal{F}(\langle b, c, d\rangle)=c \\
\mathcal{F}(\langle c, d, e\rangle)=e
\end{array}\right)\right.
$$

By false-name-proofness, whenever there are ballots for location $b$, location $c$, and location $d$ (and maybe other locations as well), the outcome cannot be location $c$, otherwise the second agent in the profile $\langle b, c, d\rangle$ would have a false-name manipulation, and it cannot be location $d$, otherwise the third agent in the profile $\langle b, c, d\rangle$ would have a false-name manipulation. Similarly, the outcome when there are ballots for location $c$, location $d$, and location $e$ (and maybe other locations as well) cannot be location $c$ or location $e$. Next, we note that by false-name-proofness,

- Whenever there are ballots only for locations $c$ and $e$, the outcome must be location $d$, otherwise one of the agents would have a false-name manipulation and cast enough false-name ballots for locations $c, d$, and $e$ and change the outcome to be location $d$, which she prefers.

- Whenever there are ballots only for locations $b$ and $c$, the outcome must be location $b$, since the agent who voted for it can change the outcome to be location $b$ by casting enough false-name ballots.

and similarly 
- Whenever there are ballots only for locations $b$ and $d$, the outcome must be location $b$.

- Whenever there are ballots only for locations $c$ and $d$, the outcome must be location $d$.

- Whenever there are ballots only for locations $d$ and $e$, the outcome must be location $d$.

Hence, we get the third order-based mechanism in the proposition.

Proposition 11 For $n \geqslant 6$ there is no anonymous Pareto-optimal mechanism for $C_{n}$ that is resistant even only to manipulations of a single agent.

Proof The proof generalizes the proof for $C_{6}$ which we showed on Proposition 3. For simplicity of notations we divide the proof into three cases (The proofs differ in the correct rounding of the locations indices needed for the integral inequalities of the proof):

Cycles of even size $n \geqslant 6$ (which was proved in Sect. 6.1),

Large cycles $\left(C_{n}\right.$ for $n \geqslant 6$ except $n=7, n=11$, and $\left.n \in\{6,8,10,12,14,16,20\}\right)$,

and last $C_{7}$ and $C_{11}$.

\section{$C_{n}$ for $\geqslant 6$ except $n=7, n=11$, and $n \in\{6,8,10,12,14,16,20\}$ :}

Assume towards a contradiction that $\mathcal{F}$ is a Pareto-optimal, anonymous, manipulationresistant mechanism for $C_{n}$. We denote the locations of $C_{n}$ by $\{0,1,2,3, \ldots, n-1\}$, and w.l.o.g. assume that for the profile of $n$ agents who vote for all $n$ locations the outcome is 0 (see Fig. 23).

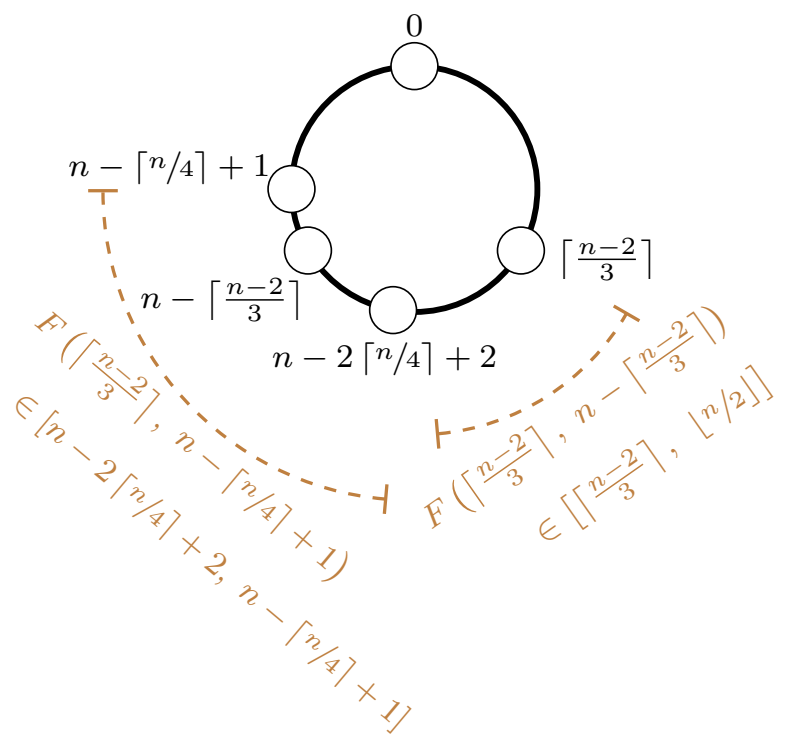

Fig. 23 Illustration for the proof of Proposition 11: second case (Color figure online) 


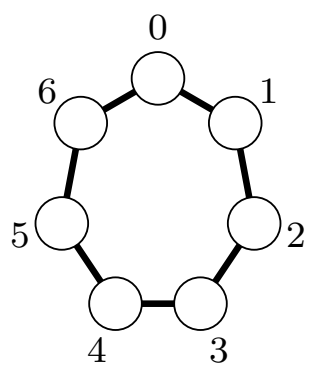

Fig. 24 The labeling of probing Proposition 11 for the cycle of size 7

For the profile $\left\langle\left\lceil\frac{n-2}{3}\right\rceil, n-\left\lceil\frac{n-2}{3}\right\rceil\right\rangle$ : From the Pareto-optimality of $\mathcal{F}$, since $n \neq 8$ and

$$
\left(n-\left\lceil\frac{n-2}{3}\right\rceil\right)-\left\lceil\frac{n-2}{3}\right\rceil<\frac{n}{2},
$$

the outcome must be in $\left[\left\lceil\frac{n-2}{3}\right\rceil, n-\left\lceil\frac{n-2}{3}\right\rceil\right]$. W.l.o.g. assume it is in $\left[\left\lceil\frac{n-2}{3}\right\rceil,\left\lfloor\frac{n}{2}\right\rfloor\right]$.

Next, we consider the profile $\left\langle\left\lceil\frac{n-2}{3}\right\rceil, n-\left\lceil\frac{n}{4}\right\rceil+1\right\rangle$ : From the Pareto-optimality of $\mathcal{F}$, since $^{24}$

$$
\left(n-\left\lceil\frac{n}{4}\right\rceil+1\right)-\left\lceil\frac{n-2}{3}\right\rceil<\frac{n}{2},
$$

the outcome must be in $\left[\left\lceil\frac{n-2}{3}\right\rceil, n-\left\lceil\frac{n}{4}\right\rceil+1\right]$. Since the second agent in this profile can change the result to be 0 by adding false-ballots, by false-name-proofness the outcome must be in $\left[n-2\left\lceil\frac{n}{4}\right\rceil+2, n-\left\lceil\frac{n}{4}\right\rceil+1\right]$. Hence, the second agent in the profile $\left\langle\left\lceil\frac{n-2}{3}\right\rceil, n-\left\lceil\frac{n-2}{3}\right\rceil\right\rangle$ (who is located on $n-\left\lceil\frac{n-2}{3}\right\rceil$ ) can change the outcome to be $\mathcal{F}\left(\left\lceil\frac{n-2}{3}\right\rceil, n-\left\lceil\frac{n}{4}\right\rceil+1\right)$ which is closer to her by changing her vote to $n-\left\lceil\frac{n}{4}\right\rceil+1$. So we get a contradiction.

$C_{7}$ :

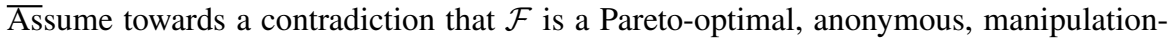
resistant mechanism for $C_{7}$. We denote the locations of $C_{7}$ by $\{0,1,2,3,4,5,6\}$, and w.l.o.g. assume that for the profile of seven agents who vote for all seven locations the outcome is 0 (see Fig. 24).

For the profile $\langle\mathbf{2}, \mathbf{5}\rangle$ : From resistance to false-name manipulations of the first and last agents, the outcome must be 0,3 , or 4 (Since any of them can change the result to be 0 by adding false-ballots). From the Pareto-optimality of $\mathcal{F}$, the outcome cannot be 0 which is Pareto-dominated by 4 . Hence, the outcome for the profile $\langle\mathbf{2}, \mathbf{5}\rangle$ is either 3 or 4 . W.1.o.g. assume it is 3 . By strategy-proofness, also the outcome for the profile $\langle\mathbf{3}, \mathbf{5}\rangle$ must be 3 (Otherwise, the first agent in this profile has a beneficial manipulation of voting 2 ).

\footnotetext{
${ }^{24}$ This inequality does not hold for $n \in\{6,7,8,10,11,12,14,16,20\}$. Hence, these values are omitted from this case.
} 


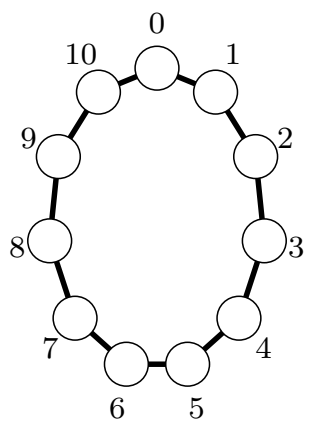

Fig. 25 The labeling of probing Proposition 11 for the cycle of size 11

Now, let us consider the profile $\langle\mathbf{3}, \mathbf{6}\rangle$ : From the Pareto-optimality of $\mathcal{F}$, the outcome cannot be 0 which is Pareto-dominated by 5 . Since the second agent in this profile can change the result to be 0 by adding false-ballots, by false-name-proofness the outcome must be either 5 or 6 . Hence, the second agent in the profile $\langle\mathbf{3}, \mathbf{5}\rangle$ (who is located on 5) can change the outcome to be $\mathcal{F}(3,6)$ which is closer to her by changing her vote to 6 . So we get a contradiction.

$C_{11}$ :

Assume towards a contradiction that $\mathcal{F}$ is a Pareto-optimal, anonymous, manipulationresistant mechanism for $C_{11}$. We denote the locations of $C_{11}$ by $\{0,1,2,3, \ldots, 10\}$, and w.l.o.g. assume that for the profile of eleven agents who vote for all eleven locations the outcome is 0 (see Fig. 25).

For the profile $\langle\mathbf{4}, \mathbf{7}\rangle$ : From the Pareto-optimality of $\mathcal{F}$, the outcome must lie in $\{4,5,6,7\}$ (The locations $0,1,2$, and 3 are Pareto-dominated by 5 . the locations 8,9 , and 10 are Pareto-dominated by 6). W.1.o.g. we assume it is either 4 or 5 .

For the profile $\langle 4,9\rangle$ : From resistance to false-name manipulations of the first and last agents, the outcome must be 0,7 , or 8 (Since any of them can change the result to be 0 by adding false-ballots). From the Pareto-optimality of $\mathcal{F}$, the outcome cannot be 0 which is Pareto-dominated by 7 . Hence, the outcome for the profile $\langle 4,9\rangle$ is either 7 or 8 .

Hence, the second agent in the profile $\langle 4,7\rangle$ (who is located on 7) can change the outcome to be $\mathcal{F}(4,9)$ which is closer to her by changing her vote to 9 . So we get a contradiction.

Acknowledgements We would like to thank Kentaro Yahiro, Nathanaël Barrot, Tomer Siedner, and Edith Elkind for their comments, and the anonymous reviewers of AAMAS and Autonomous Agents and MultiAgent Systems for their detailed reviews, which helped us to improve the presentation of this work. Preliminary versions of this work were presented by Ilan Nehama in The 14th Meeting of the Society for Social Choice and Welfare (2016), COMSOC 2018, The 7th International Workshop on Computational Social Choice, WINE 2018, the 14th Conference on Web and Internet Economics, AAMAS 2019, the 18th International Conference on Autonomous Agents and Multiagent Systems, and Haifa 1st Social Choice Workshop. We would like to thank the participants of these meetings for their comments.

Open Access This article is licensed under a Creative Commons Attribution 4.0 International License, which permits use, sharing, adaptation, distribution and reproduction in any medium or format, as long as you give appropriate credit to the original author(s) and the source, provide a link to the Creative Commons licence, and indicate if changes were made. The images or other third party material in this article are included in the article's Creative Commons licence, unless indicated otherwise in a credit line to the 
material. If material is not included in the article's Creative Commons licence and your intended use is not permitted by statutory regulation or exceeds the permitted use, you will need to obtain permission directly from the copyright holder. To view a copy of this licence, visit http://creativecommons.org/licenses/by/4.0/.

\section{References}

1. Aleskerov, F. (2002). Categories of Arrovian voting schemes. In: K. J. Arrow, A. K. Sen, and K. Suzumura (eds.), Handbook of social choice and welfare, vol. 1, chapter 2 (pp. 95-129). Elsevier.

2. Alon, N., Feldman, M., Procaccia, A. D., \& Tennenholtz, M. (2009). Strategyproof approximation mechanisms for location on networks. arXiv:0907.2049.

3. Alon, N., Feldman, M., Procaccia, A. D., \& Tennenholtz, M. (2010). Strategyproof approximation of the minimax on networks. Mathematics of Operations Research, 35(3), 513-526.

4. Aziz, H., Bachrach, Y., Elkind, E., \& Paterson, M. (2011). False-name manipulations in weighted voting games. Journal of Artificial Intelligence Research, 40, 57-93.

5. Black, D. (1948). On the rationale of group decision-making. Journal of Political Economy, 56(1), 23-34.

6. Border, K. C., \& Jordan, J. S. (1983). Straightforward elections, unanimity and phantom voters. The Review of Economic Studies, 50(1), 153-170.

7. Chan, H., Filos-Ratsikas, A., Li, B., Li, M., \& Wang, C. (2021). Mechanism design for facility location problems: A survey. In Z.-H. Zhou (eds.), Proceedings of the 13th international joint conference on artificial intelligence (IJCAI) (pp. 4356-4365).

8. Chen, Z., Fong, K. C. K., Li, M., Wang, K., Yuan, H., \& Zhang, Y. (2020). Facility location games with optional preference. Theoretical Computer Science, 847, 185-197.

9. Cheng, Y., \& Zhou, S. (2015). A survey on approximation mechanism design without money for facility games. In: D. Gao, N. Ruan, W. Xing (eds.), Advances in global optimization (pp. 117-128). Springer.

10. Cheng, Y., Wei, Yu., \& Zhang, G. (2013). Strategy-proof approximation mechanisms for an obnoxious facility game on networks. Theoretical Computer Science, 497, 154-163.

11. Church, R. L., \& Garfinkel, R. S. (1978). Locating an obnoxious facility on a network. Transportation Science, 12(2), 107-118.

12. Clarke, E. H. (1971). Multipart pricing of public goods. Public Choice, 1, 17-33.

13. Conitzer, V. (2007). Limited verification of identities to induce false-name-proofness. In: Proceedings of the 11th conference on theoretical aspects of rationality and knowledge (TARK) (pp. 102-111).

14. Conitzer, V. (2008a). Using a memory test to limit a user to one account. In: Agent-mediated electronic commerce and trading agent design and analysis-AAMAS workshop, AMEC 2008, and AAAI workshop, TADA 2008, revised selected papers (pp. 60-72). Springer.

15. Conitzer, V. (2008b). Anonymity-proof voting rules. In: Proceedings of the 4th workshop on internet and network economics (WINE) (pp. 295-306).

16. Conitzer, V., \& Yokoo, M. (2010). Using mechanism design to prevent false-name manipulations. AI Magazine, 31(4), 65-78.

17. de Ridder H. N. et al. Small graphs part of the information system on graph classes and their inclusions (ISGCI). https://www.graphclasses.org/smallgraphs.html.

18. Dokow, E., Feldman, M., Meir, R., \& Nehama, I. (2012). Mechanism design on discrete lines and cycles. In: Proceedings of the 13th ACM conference on economics and computation (EC) (pp. 423-440).

19. Douceur, J. R. (2002). The Sybil attack. In: Proceedings of the 1st international workshop peer-to-peer systems IPTPS (pp. 251-260).

20. Escoffier, B., Gourvès, L., Thang, N. K., Pascual, F., \& Spanjaard, O. (2011). Strategy-proof mechanisms for facility location games with many facilities. In: Proceedings of the 2 nd international conference on algorithmic decision theory (ADT) (pp. 67-81).

21. Farahani, R. Z., \& Hekmatfar, M. (eds) (2009). Facility location: Concepts, models, algorithms and case studies. Physica-Verlag Heidelberg, 1 edition.

22. Feigenbaum, I., \& Sethuraman, J. (2015). Strategyproof mechanisms for one-dimensional hybrid and obnoxious facility location models. In AAAI workshop: Incentive and trust in E-communities.

23. Feigenbaum, I., Sethuraman, J., \& Ye, C. (2017). Approximately optimal mechanisms for strategy proof facility location: Minimizing $L_{p}$ norm of costs. Mathematics of Operations Research, 42(2), 434-447.

24. Feldman, M., \& Wilf, Y. (2013). Strategyproof facility location and the least squares objective. In: Proceedings of the 14th ACM conference on economics and computation (EC) (pp. 873-890). 
25. Feldman, M., Fiat, A., \& Golomb, I. (2016). On voting and facility location. In: Proceedings of the 16th ACM conference on economics and computation (EC) (pp. 269-286).

26. Fishburn, P. C., \& Brams, S. J. (1983). Paradoxes of preferential voting. Mathematics Magazine, 56(4), 207-214.

27. Fong, C. K. K., Li, M., Lu, P., Todo, T., \& Yokoo, M. (2018). Facility location games with fractional preferences. In: Proceedings of the 32nd conference on artificial intelligence (AAAI) (pp. 1039-1046).

28. Fotakis, D., \& Tzamos, C. (2014). On the power of deterministic mechanisms for facility location games. ACM Transactions on Economics and Computation (TEAC), 2(4), 15:1-15:37.

29. Fotakis, D., \& Tzamos, C. (2016). Strategyproof facility location for concave cost functions. Algorithmica, 76(1), 143-167.

30. Gibbard, A. (1973). Manipulation of voting schemes: A general result. Econometrica, 41, 587-601.

31. Gibbard, A. (1977). Manipulation of schemes that mix voting with chance. Econometrica, 45(3), 665-681.

32. Groves, T. (1973). Incentives in teams. Econometrica, 41(4), 617-631.

33. Harary, F. (1963). A characterization of block-graphs. Canadian Mathematical Bulletin (CMB), 6, 1-6.

34. Ibara, K., \& Nagamochi, H. (2012). Characterizing mechanisms in obnoxious facility game. In Proceedings of the 6th conference on combinatorial optimization and applications (COCOA) (pp. 301-311).

35. Iwasaki, A., Yokoo, M., \& Terada, K. (2005). A robust open ascending-price multi-unit auction protocol against false-name bids. Decision Support Systems, 39(1), 23-39.

36. Iwasaki, A., Conitzer, V., Omori, Y., Sakurai, Y., Todo, T., Guo, M., Yokoo, M. (2010). Worst-case efficiency ratio in false-name-proof combinatorial auction mechanisms. In Proceedings of the 9th international conference on autonomous agents and multiagent systems (AAMAS) (pp. 633-640).

37. Lasisi, R. O., \& Allan, V. H. (2017). False-name manipulation in weighted voting games: Empirical and theoretical analysis. Computational Intelligence, 33(3), 478-506.

38. Lehmann, B., Lehmann, D., \& Nisan, N. (2006). Combinatorial auctions with decreasing marginal utilities. Games and Economic Behavior, 55(2), 270-296 (Mini Special Issue: Electronic Market Design.).

39. Lu, P., Wang, Y., \& Zhou, Y. (2009). Tighter bounds for facility games. In: Proceedings of the 5th workshop on internet and network economics (WINE) (pp. 137-148).

40. Lu, P., Sun, X., Wang, Y., Zhu, Z. A. (2010). Asymptotically optimal strategy-proof mechanisms for two-facility games. In Proceedings of the 11th ACM conference on economics and computation (EC) (pp. $315-324)$.

41. Mas-Colell, A., Whinston, M., \& Green, J. (1995). Microeconomic theory. Oxford: Oxford University Press.

42. Moulin, H. (1980). On strategy-proofness and single peakedness. Public Choice, 35(4), 437-455.

43. Moulin, Hervé. (1988). Condorcet's principle implies the no show paradox. Journal of Economic Theory, 45(1), 53-64.

44. Moulin, H. (1995). Cooperative Microeconomics: A Game-Theoretic Introduction. Princeton: Princeton University Press.

45. Moulin, H. (2014). Pricing traffic in a spanning network. Games and Economic Behavior, 86, 475-490.

46. Myerson, R. B. (1979). Incentive compatibility and the bargaining problem. Econometrica, 47, 61-74.

47. Nehama, I. Almost quasi-linear utilities in disguise: An extension of Roberts' theorem (work in progress).

48. Nehama, I. (2019). Almost quasi-linear utilities in disguise: Positive-representation an extension of Roberts' theorem. In Proceedings of the 15th conference on web and internet economics (WINE) (pp. 344-345).

49. Nehama, I., Todo, T., \& Yokoo, M. (2019). Manipulation-resistant facility location mechanisms for $Z V$-line graphs. In: Proceedings of the 18th international conference on autonomous agents and multiagent systems (AAMAS) (pp. 1452-1460).

50. Okada, N., Todo, T., \& Yokoo, M. (2019). SAT-based automated mechanism design for false-nameproof facility location. In: Proceedings of the $22 \mathrm{nd}$ principles and practice of multi-agent systems (PRIMA) (pp. 321-337).

51. Ono, T., Todo, T., \& Yokoo, M. (2017). Rename and false-name manipulations in discrete facility location with optional preferences. In: Proceedings of the 20th principles and practice of multiagent systems (PRIMA) (pp. 163-179). 
52. Penna, P., Schoppmann, F., Silvestri, R., \& Widmayer, P. (2009). Pseudonyms in cost-sharing games. In: Proceedings of the 5th workshop on internet and network economics (WINE) (pp. 256-267).

53. Procaccia, A. D., \& Tennenholtz, M. (2013). Approximate mechanism design without money. ACM Transactions on Economics and Computation (TEAC), 1(4).

54. Rastegari, B., Condon, A., \& Leyton-Brown, K. (2011). Revenue monotonicity in deterministic, dominant-strategy combinatorial auctions. Artificial Intelligence, 175(2), 441-456.

55. Roberts, K. (1979). The characterization of implementable choice rules. In: J.-J. Laffont (eds.), Aggregation and revelation of preferences (pp. 321-349). North-Holland.

56. Sakurai, Y., Yokoo, M. (2002). An average-case budget-non-negative double auction protocol. In: Proceedings of the 1st international conference on autonomous agents and multiagent systems (AAMAS) (pp. 104-111).

57. Sakurai, Y., Yokoo, M. (2003). A false-name-proof double auction protocol for arbitrary evaluation values. In: Proceedings of the 2nd international conference on autonomous agents and multiagent systems (AAMAS) (pp. 329-336).

58. Sandholm, T. (2003). Automated mechanism design: A new application area for search algorithms. In: Proceedings of the principles and practice of constraint programming (CP) (pp. 19-36).

59. Satterthwaite, M. A. (1975). Strategy-proofness and Arrow's conditions: Existence and correspondence theorems for voting procedures and social welfare functions. Journal of Economic Theory, 10, $187-217$.

60. Schummer, J., \& Vohra, R. V. (2002). Strategy-proof location on a network. Journal of Economic Theory, 104(2), 405-428.

61. Serafino, P., \& Ventre, C. (2016). Heterogeneous facility location without money. Theoretical Computer Science, 636, 27-46.

62. Sonoda, A., Todo, T., \& Yokoo, M. (2016). False-name-proof locations of two facilities: Economic and algorithmic approaches. In: Proceedings of the 30th conference on artificial intelligence (AAAI) (pp. 615-621).

63. Suyama, T., \& Yokoo, M. (2005). Strategy/false-name proof protocols for combinatorial multiattribute procurement auction. Autonomous Agents and Multi-Agent Systems, 11(1), 7-21.

64. Terada, K., \& Yokoo, M. (2006). False-name-proof multi-unit auction protocol utilizing greedy allocation based on approximate evaluation values. Systems and Computers in Japan, 37(13), 89-98.

65. Todo, T., \& Conitzer, V. (2013). False-name-proof matching. In Proceedings of the 12th international conference on autonomous agents and multiagent systems (AAMAS) (pp. 311-318).

66. Todo, T., Iwasaki, A., Yokoo, M., \& Sakurai, Y. (2009). Characterizing false-name-proof allocation rules in combinatorial auctions. In: Proceedings of the 8th international conference on autonomous agents and multiagent systems (AAMAS) (pp. 265-272).

67. Todo, T., Iwasaki, A., \& Yokoo, M. (2011). False-name-proof mechanism design without money. In: Proceedings of the 10th international conference on autonomous agents and multiagent systems (AAMAS) (pp. 651-658).

68. Todo, T., Mouri, T., Iwasaki, A., \& Yokoo, M. (2012). False-name-proofness in online mechanisms. In: Proceedings of the 11th international conference on autonomous agents and multiagent systems (AAMAS) (pp. 753-762).

69. Todo, T., Okada, N., \& Yokoo, M. (2020). False-name-proof facility location on discrete structures. In: Proceedings of the 24th European conference on artificial intelligence (ECAI) (pp. 227-234).

70. Tsuruta, S., Oka, M., Todo, T., Kawasaki, Y., Guo, M., Sakurai, Y., \& Yokoo, M. (2014). Optimal false-name-proof single-item redistribution mechanisms. In Proceedings of the 13th international conference on autonomous agents and multiagent systems (AAMAS) (pp. 221-228).

71. Tsuruta, S., Oka, M., Todo, T., Sakurai, Y., \& Yokoo, M. (2015). Fairness and false-name manipulations in randomized cake cutting. In Proceedings of the 14th international conference on autonomous agents and multiagent systems (AAMAS) (pp. 909-917).

72. Vickrey, W. (1961). Counterspeculation, auctions, and competitive sealed tenders. The Journal of Finance, 16(1), 8-37.

73. Wada, Y., Ono, T., Todo, T., \& Yokoo, M. (2018). Facility location with variable and dynamic populations. In Proceedings of the 17th international conference on autonomous agents and multiagent systems (AAMAS) (pp. 336-344).

74. Wagman, L., \& Conitzer, V. (2008). Optimal false-name-proof voting rules with costly voting. In: Proceedings of the 23rd conference on artificial intelligence (AAAI) (pp. 190-195).

75. Wagman, L., \& Conitzer, V. (2014). False-name-proof voting with costs over two alternatives. International Journal of Game Theory, 43(3), 599-618. 
76. Yokoo, M. (2003). Characterization of strategy/false-name proof combinatorial auction protocols: Price-oriented, rationing-free protocol. In: Proceedings of the 8th international joint conference on artificial intelligence (IJCAI) (pp. 733-742).

77. Yokoo, M., Sakurai, Y., \& Matsubara, S. (2001a). Robust multi-unit auction protocol against falsename bids. In: Proceedings of the 7th international joint conference on artificial intelligence (IJCAI) (pp. 1089-1094).

78. Yokoo, M., Sakurai, Y., \& Matsubara, S. (2001). Robust combinatorial auction protocol against falsename bids. Artificial Intelligence, 130(2), 167-181.

79. Yokoo, M., Sakurai, Y., \& Matsubara, S. (2004). The effect of false-name bids in combinatorial auctions: New fraud in internet auctions. Games and Economic Behavior, 46(1), 174-188.

80. Yokoo, M., Sakurai, Y., \& Matsubara, S. (2005). Robust double auction protocol against false-name bids. Decision Support Systems, 39(2), 241-252.

81. Zhao, D., Luo, S., Todo, T., \& Yokoo, M. (2014). False-name-proof combinatorial auction design via single-minded decomposition. In Proceedings of the 21st European conference on artificial intelligence (ECAI) (pp. 945-950).

82. Zou, S., \& Li, M. (2015). Facility location games with dual preference. In Proceedings of the 14th international conference on autonomous agents and multiagent systems (AAMAS) (pp. 615-623).

Publisher's Note Springer Nature remains neutral with regard to jurisdictional claims in published maps and institutional affiliations.

\title{
Authors and Affiliations
}

\author{
Ilan Nehama ${ }^{1,3}$ (D) Taiki Todo $^{2}$ (D) $\cdot$ Makoto Yokoo ${ }^{2}$ \\ Taiki Todo \\ todo@inf.kyushu-u.ac.jp \\ Makoto Yokoo \\ yokoo@inf.kyushu-u.ac.jp \\ 1 Bar-Ilan University, Ramat Gan, Israel \\ 2 Kyushu University, Fukuoka, Japan \\ 3 Western Galilee College, Akko, Israel
}

